Research Article

\title{
Classifying multiplets of totally real cubic fields
}

\author{
Daniel C. Mayer*, $\dagger$ \\ Naglergasse 53, 8010 Graz, Austria
}

(Received: 20 April 2021. Received in revised form: 12 July 2021. Accepted: 16 July 2021. Published online: 19 July 2021.)

(C) 2021 the author. This is an open access article under the CC BY (International 4.0) license (www.creativecommons.org/licenses/by/4.0/).

\begin{abstract}
The number of non-isomorphic cubic fields $L$ sharing a common discriminant $d_{L}=d$ is called the multiplicity $m=m(d)$ of $d$. For an assigned value of $d$, these fields are collected in a homogeneous multiplet $\mathbf{M}_{d}=\left(L_{1}, \ldots, L_{m}\right)$. By entirely new techniques for the construction and classification, we determine the differential principal factorization types $\tau\left(L_{i}\right) \in$ $\left\{\alpha_{1}, \alpha_{2}, \alpha_{3}, \beta_{1}, \beta_{2}, \gamma, \delta_{1}, \delta_{2}, \varepsilon\right\}$ of the members $L_{i}$ of each multiplet $\mathbf{M}_{d}$ of non-cyclic totally real cubic fields with discriminants $d<10^{7}$. This is a new kind of arithmetical invariants which provide succinct information about ambiguous principal ideals and capitulation in the normal closures $N$ of non-Galois cubic fields $L$. The classification is arranged with respect to increasing 3-class rank $\varrho$ of the quadratic subfields $K$ of the $S_{3}$-fields $N$, and to ascending number of prime divisors of the conductor $f$ of $N / K$. The Scholz conjecture concerning the distinguished index of subfield units $\left(U_{N}: U_{0}\right)=1$ for ramified extensions $N / K$ with conductor $f>1$ is also verified by a tremendous minimal discriminant $d=18251060$ outside of the systematic range $d<10^{7}$.
\end{abstract}

Keywords: 3-ring class fields; 3-admissible conductors; quadratic base fields; non-Galois triply real cubic fields; totally real $S_{3}$-fields; dihedral fields; multiplicity of discriminants; 3-Selmer space; 3-ring spaces; multiplets; Galois cohomology; differential principal factorizations; capitulation of 3-class groups; statistics; Scholz conjecture.

2020 Mathematics Subject Classification: 11R37, 11R11, 11R16, 11R20, 11R27, 11R29, $11 Y 40$.

\section{Introduction}

We present entirely new techniques for the construction and classification of non-Galois cubic fields $L$ as subfields $L<K_{f}$ of a 3-ring class field $K_{f}$ modulo a 3-admissible conductor $f$ over a quadratic field $K$. In [35], we used the new methods to classify all complex and totally real cubic fields $L$ with discriminants $-2 \cdot 10^{4}<d_{L}<10^{5}$, computed between 1972 and 1976 by Angell [1,2].

In this paper, we omit simply real cubic fields with a few types only, and we rather put our focus on triply real cubic fields $L$ with nine possible types $\tau(L) \in\left\{\alpha_{1}, \alpha_{2}, \alpha_{3}, \beta_{1}, \beta_{2}, \gamma, \delta_{1}, \delta_{2}, \varepsilon\right\}$, which refine the coarse classification with five types $\alpha, \beta, \gamma, \delta, \varepsilon$ by Moser [36]. According to the historical development of systematic investigations, we arrange our refined classification of homogeneous multiplets $\mathbf{M}_{d}$ of totally real cubic fields $L$ in four steps with increasing upper bounds $B \leq 10^{7}$ for the discriminants $d_{L}<B$.

In Section 4, we summarize our results reported in [35] concerning the range $d_{L}<10^{5}$ of Angell [1,2]. In Section 5 , we continue with an update of our extension to $d_{L}<2 \cdot 10^{5}$ given in [26,27], which was computed in 1991 by means of the Voronoi algorithm [40]. Whereas the count of discriminants and fields and the collection of fields into multiplets were correct, the classification into type $\delta_{2}$ instead of $\beta_{2}$ was partially erroneous, because absolute principal factors do not necessarily show up among the lattice minima in the chains of Voronoi's algorithm. The highlights of this paper will be established in Sections 6 and 7, where we use our new techniques employing Fieker's class field routines [11] in Magma [25] to classify the range $d_{L}<5 \cdot 10^{5}$ of Ennola and Turunen [9,10] and the range $d_{L}<10^{7}$ of Llorente and Quer [24], the most extensive ranges deposited in files of unpublished mathematical tables (UMT). This coronation expands our restricted investigations [29,32] of unramified normal closures $N / K$ (absolute Hilbert 3-class fields) to all ramified cases $N / K$ with arbitrary conductors $f \geq 1$ (3-ring class fields). Statistical evaluations and theoretical conclusions are given in Section 8.

We mention that Belabas [5] has given a fast method for simply counting cubic fields even in bigger ranges, without computation of arithmetical invariants, like fundamental systems of units and class group structures, and without classification into differential principal factorization types.

\footnotetext{
*E-mail address: algebraic.number.theory@algebra.at

$\dagger$ †copus Author Profile: https : //www. scopus . com/authid/detail . uri ?authorId=36696761400
} 
The latter are introduced in Section 3 , where we prove that the $\mathbb{F}_{p}$-vector space $\mathcal{P}_{N / K} / \mathcal{P}_{K}$ of primitive ambiguous principal ideals of a number field extension $N / K$ with odd prime degree $p$ can be endowed with a natural trichotomic direct product structure and corresponding invariants $(A, R, C)$.

Finally, as an application of the notions of multiplets and DPF types, the Scholz conjecture [39] concerning the distinguished index of subfield units $\left(U_{N}: U_{0}\right)=1$ of the normal closure $N$ of $L$ is stated, refined, and proved completely in Section 9 .

We clarify in advance why we present the results of our classification for several intervals $0<d_{L}<B$ of positive cubic discriminants instead of focussing on the most extensive of these ranges: Firstly, we want to add value to the classical tables of Angell, Ennola and Turunen, Llorente and Quer, which did not illuminate the constitution of multiplets $\left(L_{1}, \ldots, L_{m}\right)$ of totally real cubic fields as subfields of a common 3-ring class field $K_{f}$ modulo a 3-admissible conductor $f$ over a quadratic base field $K$, let alone the differential principal factorization types $\left(\tau\left(L_{1}\right), \ldots, \tau\left(L_{m}\right)\right)$. Secondly, it is our intention to show the increasing wealth of arithmetical structure in three successive extensions of the upper bound $B$ from $10^{5}$ to $2 \cdot 10^{5}, 5 \cdot 10^{5}$, and finally $10^{7}$. There arise increasing 3-class ranks $\varrho$ of $K$, conductors $f$ divisible by an ascending number of primes, new types $\tau(L)$, and heterogeneous multiplets $\mathbf{M}\left(K_{f}\right)=\left[\mathbf{M}_{c^{2} d}\right]_{c \mid f}=\left[\left(L_{c, 1}, \ldots, L_{c, m(c)}\right)\right]_{c \mid f}$ with increasing complexity.

\section{Construction as subfields of a ring class field}

\subsection{Structure and multiplicity of cubic discriminants}

Let $K$ be a quadratic number field with fundamental discriminant $d=d_{K}$ (square free, except possibly for the 2-contribution).

Definition 2.1. A positive integer $f$ is called a 3-admissible conductor for $K$, if it has the shape $f=3^{e} \cdot q_{1} \cdots q_{t}$ with an integer exponent $e \in\{0,1,2\}, t \geq 0$, and pairwise distinct prime numbers $q_{1}, \ldots, q_{t} \in \mathbb{P} \backslash\{3\}$, such that the following conditions are satisfied:

$$
\text { Kronecker symbol }\left(\frac{d}{q_{i}}\right) \equiv q_{i}(\bmod 3), \text { for all } 1 \leq i \leq t,
$$

and

$$
e \in \begin{cases}\{0,2\} & \text { if } d \equiv \pm 1(\bmod 3) \\ \{0,1\} & \text { if } d \equiv+3(\bmod 9) \\ \{0,1,2\} & \text { if } d \equiv-3(\bmod 9)\end{cases}
$$

So, a 3-admissible conductor $f$ for $K$ is essentially square free, except possibly for the critical contribution by the prime 3 . The condition involving the Kronecker symbol means that a non-critical prime divisor $q \neq 3$ of $f$ must remain inert in $K$, if $q \equiv-1(\bmod 3)$, and must split in $K$, if $q \equiv+1(\bmod 3)$. The critical prime divisor 3 of $f$ is 3 -admissible if and only if it ramifies in $K$, that is, if $d \equiv \pm 3(\bmod 9)$. Otherwise only the critical prime power divisor 9 of $f$ is 3-admissible. (Recall that 3 remains inert in $K$ if $d \equiv-1(\bmod 3)$ and 3 splits in $K$ if $d \equiv+1(\bmod 3)$.) So far, all contributions to $f$ are regular. There is, however, the possibility of an irregular 3 -admissible critical prime power divisor 9 of $f$, when $d \equiv-3(\bmod 9)$.

Definition 2.2. An integer $D=f^{2} \cdot d$ is called a formal cubic discriminant if $f$ is a 3-admissible conductor for the quadratic field $K$ with fundamental discriminant $d$. (Since the square $f^{2}$ and the fundamental discriminant d are congruent to 0 or 1 modulo 4 , this is also the case for a formal cubic discriminant $D$. We shall see that D is not necessarily discriminant $d_{L}$ of a cubic field L.)

Note that this definition does not include discriminants $d_{L}$ of cyclic cubic fields $L$ which are perfect squares $f^{2}$ of conductors $f$ exactly divisible by primes congruent to 1 modulo 3 , and possibly also by the prime power $3^{2}$. In order to determine the multiplicity of $d_{L}$, we need further definitions.

Definition 2.3. An algebraic number $\alpha \neq 0$ in the quadratic field $K$ is called a 3-virtual unit, if its principal ideal $\alpha \mathcal{O}_{K}$ is the cube $\mathrm{j}^{3}$ of an ideal $\mathrm{j}$ of $K$. Obviously all units $\eta$ in $U_{K}$ and all third powers $\alpha^{3} \neq 0$ in $\left(K^{\times}\right)^{3}$ are 3-virtual units of $K$. Let I denote the group of all 3-virtual units of $K$, and let $K^{\times}=K \backslash\{0\}$ denote the multiplication group of $K$. The $\mathbb{F}_{3}$-vector space $V:=I /\left(K^{\times}\right)^{3}$ is called the 3-Selmer space of $K$.

For any positive integer $n$ and a set $X$ of algebraic numbers, let $X(n)$ be the subset of $X$ consisting of elements coprime to $n$. The 3-Selmer space $V$ of $K$ is isomorphic to the direct product of the 3-elementary class group $\mathrm{Cl}_{K} / \mathrm{Cl}_{K}^{3}$ and the 3-elementary unit group $U_{K} / U_{K}^{3}$ of $K$ [31, p. 2212]. Since any ideal class contains an ideal coprime to an assigned positive integer $n$, it follows that $\mathrm{Cl}_{K}=\mathcal{I}_{K} / \mathcal{P}_{K} \simeq \mathcal{I}_{K}(n) / \mathcal{P}_{K}(n)$, and since trivially $U_{K}=U_{K}(n)$, we have $V \simeq I(n) / K(n)^{3}[31$, Dfn. 2.2, p. 2211]. 
Definition 2.4. Let $f$ be a positive integer. Denote by $S_{f}:=\{\alpha \in K \mid \alpha \equiv 1(\bmod f)\}$ the ray modulo $f$ of $K$, and by $R_{f}:=\mathbb{Q}(f) \cdot S_{f}$ the ring modulo $f$ of $K$. The subspace $V(f):=\left(I(f) \cap R_{f} \cdot K(f)^{3}\right) / K(f)^{3}$ of the 3-Selmer space $V$ is called the 3-ring space modulo $f$ of $K$. Its codimension $\delta(f):=\operatorname{codim}(V(f))=\operatorname{dim}_{\mathbb{F}_{3}}(V / V(f))$ is called the 3-defect of $f$ with respect to $K$.

In order to enable comparison and binary operations (in particular, intersection) of two different ring spaces $V(f)$ and $V\left(f^{\prime}\right)$, we need the concept of a modulus of declaration, that is a positive integer $n$ which is a common multiple of $f$ and $f^{\prime}$. Then $V(f) \simeq\left(I(n) \cap R_{f} \cdot K(n)^{3}\right) / K(n)^{3}$ and $V\left(f^{\prime}\right) \simeq\left(I(n) \cap R_{f^{\prime}} \cdot K(n)^{3}\right) / K(n)^{3}$, whence it makes sense to speak about inclusion and meet.

Remark 2.1. It is possible to avoid the requirement of a modulus of declaration, if the theory of ring spaces is based on the approach via idèle groups. This has been done by Satgé [37] for prime conductors $f=q$ and will be expanded further by ourselves for any $f$ in a future paper.

\subsection{Homogeneous and heterogeneous multiplets}

If $f$ is a 3-admissible conductor with 3-defect $\delta(f)$ for a quadratic field $K$ with fundamental discriminant $d$ and 3-class rank $\varrho$, then the sum of all multiplicities $m(D)$ of formal cubic discriminants $D=c^{2} d$ with $c$ running over all divisors of $f$ is given by

$$
\sum_{c \mid f} m\left(c^{2} d\right)=\frac{1}{2}\left(3^{e_{f}}-1\right)
$$

in terms of the 3-ring class rank modulo $f$ of $K$ [31, Theorem 2.1, p. 2213],

$$
\varrho_{f}=\varrho+t+w-\delta(f),
$$

where $t:=\#\left\{q \in \mathbb{P} \backslash\{3\} \mid v_{q}(f)=1\right\}$, and $w$ is defined in terms of the 3-valuation $v_{3}(f)$ of $f$,

$$
w:= \begin{cases}0 & \text { if } v_{3}(f)=0 \\ 1 & \text { if } v_{3}(f)=1 \text { or }\left[v_{3}(f)=2 \text { and } d \equiv \pm 1(\bmod 3)\right], \\ 2 & \text { if } v_{3}(f)=2 \text { and } d \equiv 6(\bmod 9) .\end{cases}
$$

Definition 2.5. Let $f$ be a 3-admissible conductor for a quadratic field $K$.

1. For each divisor $c$ of $f$ which is also a 3-admissible conductor for $K$, the multiplet

$$
\mathbf{M}_{c^{2} d}:=\left(L_{c, 1}, \ldots, L_{c, m}\right) \text { with } m=m\left(c^{2} d\right)
$$

is called the homogeneous multiplet of cubic fields $L_{c, i}$ with discriminant $c^{2} d$.

2. The multiplet $\mathbf{M}\left(K_{f}\right):=\left[\mathbf{M}_{c^{2}}\right]_{c \mid f}$ is called the heterogeneous multiplet of the 3-ring class field $K_{f}$ modulo $f$ of $K$. (The normal closures of all cubic fields $L_{c, i}$ with $c \mid f$ and $1 \leq i \leq m\left(c^{2} d\right)$ are subfields of the ring class field $K_{f}$.)

3. The family $\operatorname{sgn}\left(\mathbf{M}\left(K_{f}\right)\right):=\left[m\left(c^{2} d\right)\right]_{c \mid f}$ of all partial multiplicities associated with $f$ is called the signature of the heterogeneous multiplet $\mathbf{M}\left(K_{f}\right)$.

$D=c^{2} d$ is only a formal but not an actual cubic discriminant if and only if the multiplicity $m\left(c^{2} d\right)=0$ vanishes, that is, if $\mathbf{M}_{c^{2} d}=\emptyset$ is a nilet (denoted by the empty set symbol).

Definition 2.6. By the type of the 3-ring class field $K_{f}$ modulo $f$ of $K$ we understand the following pair $\left(\operatorname{Obj}\left(K_{f}\right), \operatorname{Inv}\left(K_{f}\right)\right)$ of heterogeneous multiplets

$$
\begin{aligned}
\operatorname{Obj}\left(K_{f}\right) & :=\mathbf{M}\left(K_{f}\right)=\left[\left(L_{c, i}\right)_{1 \leq i \leq m\left(c^{2} d\right)}\right]_{c \mid f} \\
\operatorname{Inv}\left(K_{f}\right) & :=\tau\left(\mathbf{M}\left(K_{f}\right)\right)=\left[\left(\tau\left(L_{c, i}\right)\right)_{1 \leq i \leq m\left(c^{2} d\right)}\right]_{c \mid f}
\end{aligned}
$$

consisting of all non-cyclic cubic fields $L_{c, i}$ with discriminants $c^{2} d$ dividing $f^{2} d$ as objects and their differential principal factorization types $\tau\left(L_{c, i}\right)$ as invariants.

(See [33,34] and the next section Section 3.) 


\subsection{Algorithmic process of construction}

The computational technique which will be employed for the construction of totally real cubic fields in the sections Sections 4, 5, 6, and 7 consists of two steps. For an assigned real quadratic field $K$ with fundamental discriminant $d$ and a 3admissible conductor $f$ for $K$, initially all cyclic cubic extensions $N / K$ with conductor $f$ are constructed as subfields of the ray class field modulo $f$ of $K$. Then the members $N$ of this family are tested for their absolute automorphism group $G=\operatorname{Gal}(N / \mathbb{Q})$, and only those with $G \simeq S_{3}$ are permitted to pass the filter. As a double check, we additionally make sure that the non-Galois subfields $L<N$ have the required discriminant $d_{L}=f^{2} \cdot d$, and thus $N$ is subfield of $K_{f}$, the ring class field modulo $f$ of $K$, which is contained in the ray class field modulo $f$ of $K$. The result is the multiplet $\mathbf{M}_{f^{2} d}$, because the fields $N$ are certainly not subfields of $K_{c}$ for proper divisors $c$ of $f$.

Before we apply this algorithm, however, we have to introduce the concept of differential principal factorizations (DPF) in section Section 3.

\section{Differential principal factorization types}

Our intention in this section is to prepare sound foundations for the concept of differential principal factorization (DPF) types and to establish a common theoretical framework for the classification

- of dihedral fields $N / \mathbb{Q}$ of degree $2 p$ with an odd prime $p$, viewed as subfields of suitable $p$-ring class fields over a quadratic field $K$ (see the left part of Figure 1), and

- of pure metacyclic fields $N=K(\sqrt[p]{D})$ of degree $(p-1) \cdot p$ with an odd prime $p$, viewed as Kummer extensions of a cyclotomic field $K=\mathbb{Q}\left(\zeta_{p}\right)$ (see the right part of Figure 1),

by the following arithmetical invariants:

1. the $\mathbb{F}_{p}$-dimensions of subspaces of the space $\mathcal{P}_{N / K} / \mathcal{P}_{K}$ of primitive ambiguous principal ideals, which are also called differential principal factors, of $N / K$,

2. the capitulation kernel $\operatorname{ker}\left(T_{N / K}\right)$ of the transfer homomorphism $T_{N / K}: \mathrm{Cl}_{p}(K) \rightarrow \mathrm{Cl}_{p}(N), \mathfrak{a} \mathcal{P}_{K} \mapsto\left(\mathfrak{a} \mathcal{O}_{N}\right) \mathcal{P}_{N}$, of $p$-classes from $K$ to $N$, and

3. the Galois cohomology $\hat{\mathrm{H}}^{0}\left(G, U_{N}\right), \mathrm{H}^{1}\left(G, U_{N}\right)$ of the unit group $U_{N}$ as a module over the cyclic automorphism group $G=\operatorname{Gal}(N / K) \simeq C_{p}$.

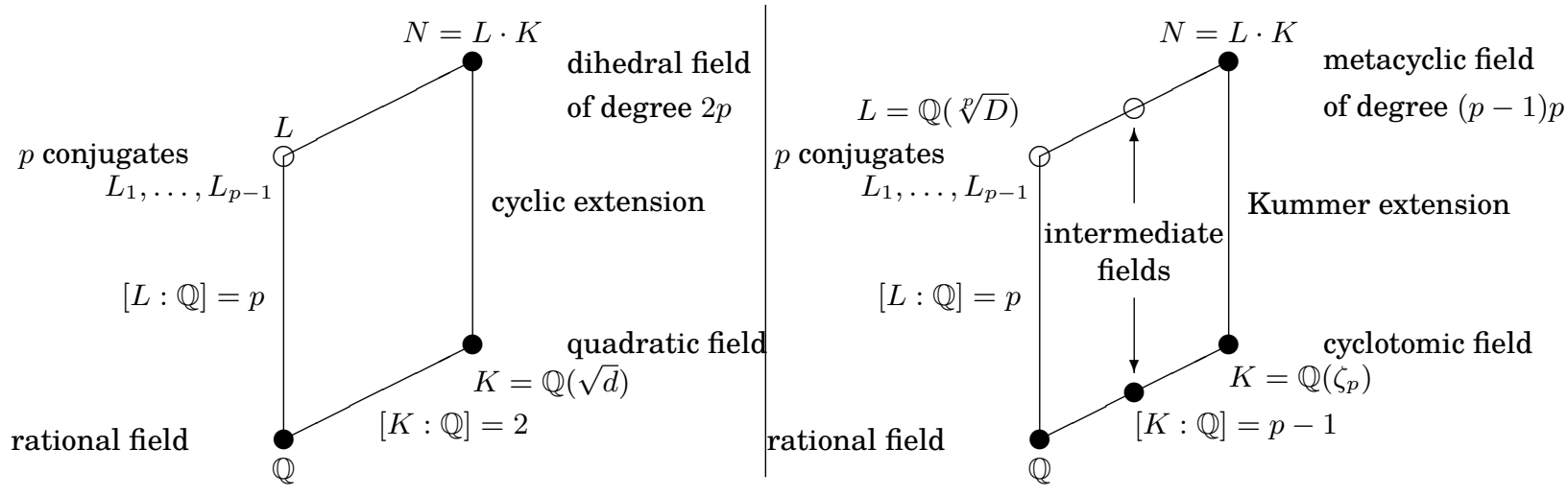

Figure 1: Dihedral and metacyclic situation.

\subsection{Primitive ambiguous ideals}

Let $p \geq 2$ be a prime number, and $N / K$ be a relative extension of number fields with degree $p$ (not necessarily Galois).

Definition 3.1. The group $\mathcal{I}_{N}$ of fractional ideals of $N$ contains the subgroup of ambiguous ideals of $N / K$, denoted by the symbol $\mathcal{I}_{N / K}:=\left\{\mathfrak{A} \in \mathcal{I}_{N} \mid \mathfrak{A}^{p} \in \mathcal{I}_{K}\right\}$. The quotient $\mathcal{I}_{N / K} / \mathcal{I}_{K}$ is called the $\mathbb{F}_{p}$-vector space of primitive ambiguous ideals of N/K. (Cfr. [33, Dfn. 3.1, p. 1991].) 
Proposition 3.1. Let $\mathfrak{L}_{1}, \ldots, \mathfrak{L}_{t}$ be the totally ramified prime ideals of $N / K$, then a basis and the dimension of the space $\mathcal{I}_{N / K} / \mathcal{I}_{K}$ over $\mathbb{F}_{p}$ are finite and given by

$$
\mathcal{I}_{N / K} / \mathcal{I}_{K} \simeq \prod_{i=1}^{t}\left(\left\langle\mathfrak{L}_{i}\right\rangle /\left\langle\mathfrak{L}_{i}^{p}\right\rangle\right) \simeq \mathbb{F}_{p}^{t}, \quad \operatorname{dim}_{\mathbb{F}_{p}}\left(\mathcal{I}_{N / K} / \mathcal{I}_{K}\right)=t
$$

whereas $\mathcal{I}_{N / K}$ is an infinite Abelian group containing $\mathcal{I}_{K}$.

Proof. According to the definition of $\mathcal{I}_{N / K}$, the quotient $\mathcal{I}_{N / K} / \mathcal{I}_{K}$ is an elementary Abelian $p$-group. By the decomposition law for prime ideals of $K$ in $N$, the space $\mathcal{I}_{N / K} / \mathcal{I}_{K}$ is generated by the totally ramified prime ideals (with ramification index $e=p$ ) of $N / K$, that is to say $\mathcal{I}_{N / K}=\left\langle\mathfrak{L} \in \mathbb{P}_{N} \mid \mathfrak{L}^{p} \in \mathbb{P}_{K}\right\rangle \mathcal{I}_{K}$. According to the theorem on prime ideals dividing the discriminant, the number $t$ of totally ramified prime ideals $\mathfrak{L}_{1}, \ldots, \mathfrak{L}_{t}$ of $N / K$ is finite.

If $L$ is another subfield of $N$ such that $N=L \cdot K$ is the compositum of $L$ and $K$, and $N / L$ is of degree $q$ coprime to $p$, then the relative norm homomorphism $N_{N / L}$ induces an epimorphism

$$
N_{N / L}: \mathcal{I}_{N / K} / \mathcal{I}_{K} \rightarrow \mathcal{I}_{L / F} / \mathcal{I}_{F},
$$

where $F:=L \cap K$ denotes the intersection of $L$ and $K$ in Figure 2. Thus, by the isomorphism theorem (see also [33, Theorem 4.2, pp. 1995-1996]), we have proved:

Theorem 3.1. There are the following two isomorphisms between finite $\mathbb{F}_{p}$-vector spaces:

$$
\begin{aligned}
\left(\mathcal{I}_{N / K} / \mathcal{I}_{K}\right) / \operatorname{ker}\left(N_{N / L}\right) & \simeq \mathcal{I}_{L / F} / \mathcal{I}_{F} \quad \text { (quotient) }, \\
\mathcal{I}_{N / K} / \mathcal{I}_{K} & \left.\simeq\left(\mathcal{I}_{L / F} / \mathcal{I}_{F}\right) \times \operatorname{ker}\left(N_{N / L}\right) \quad \text { (direct product }\right) .
\end{aligned}
$$

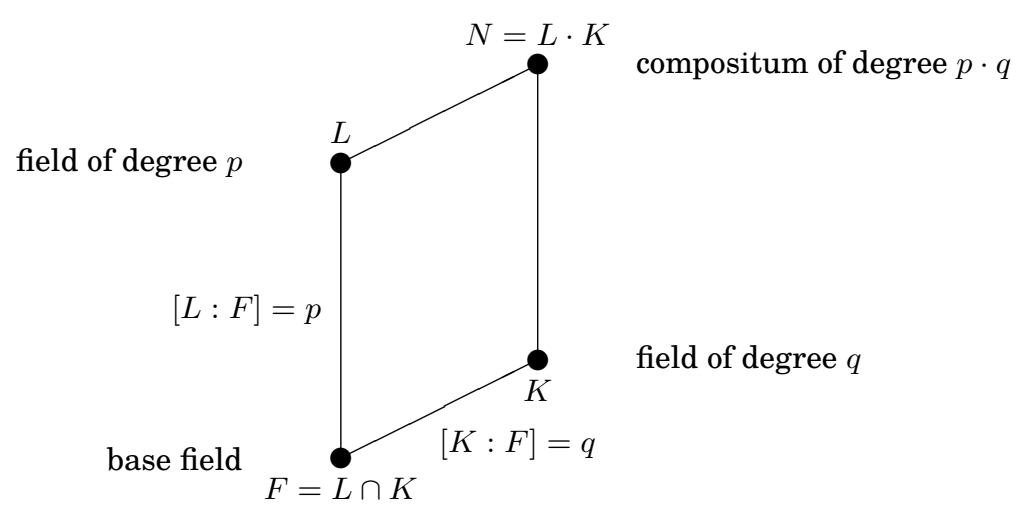

Figure 2: Hasse subfield diagram of $N / F$.

Definition 3.2. Since the relative different of $N / K$ is essentially given by $\mathfrak{D}_{N / K}=\prod_{i=1}^{t} \mathfrak{L}_{i}^{p-1}$ [33, Theorem 3.2, $p$. 1993], the space $\mathcal{I}_{N / K} / \mathcal{I}_{K} \simeq \prod_{i=1}^{t}\left(\left\langle\mathfrak{L}_{i}\right\rangle /\left\langle\mathfrak{L}_{i}^{p}\right\rangle\right)$ of primitive ambiguous ideals of $N / K$ is also called the space of differential factors of $N / K$. The two subspaces in the direct product decomposition of $\mathcal{I}_{N / K} / \mathcal{I}_{K}$ in formula (4) are called

- subspace $\mathcal{I}_{L / F} / \mathcal{I}_{F}$ of absolute differential factors of $L / F$, and

- subspace $\operatorname{ker}\left(N_{N / L}\right)$ of relative differential factors of $N / K$.

\subsection{Splitting off the norm kernel}

The second isomorphism in formula (4) gives rise to a dichotomic decomposition of the space $\mathcal{I}_{N / K} / \mathcal{I}_{K}$ of primitive ambiguous ideals of $N / K$ into two components, whose dimensions can be given under the following conditions:

Theorem 3.2. Let $p \geq 3$ be an odd prime and put $q=2$. Among the prime ideals of $L$ which are totally ramified over $F$, denote by $\mathfrak{p}_{1}, \ldots, \mathfrak{p}_{s}$ those which split in $N, \mathfrak{p}_{i} \mathcal{O}_{N}=\mathfrak{P}_{i} \mathfrak{P}_{i}^{\prime}$ for $1 \leq i \leq s$, and by $\mathfrak{q}_{1}, \ldots, \mathfrak{q}_{n}$ those which remain inert in $N, \mathfrak{q}_{j} \mathcal{O}_{N}=\mathfrak{Q}_{j}$ for $1 \leq j \leq n$. Then the space $\mathcal{I}_{N / K} / \mathcal{I}_{K}$ of primitive ambiguous ideals of $N / K$ is the direct product of the subspace $\mathcal{I}_{L / F} / \mathcal{I}_{F}$ of absolute differential factors of $L / F$ and the subspace $k e r\left(N_{N / L}\right)$ of relative differential factors 
of $N / K$, whose bases and dimensions over $\mathbb{F}_{p}$ are given by

$$
\begin{aligned}
\mathcal{I}_{L / F} / \mathcal{I}_{F} & \simeq \prod_{i=1}^{s}\left(\left\langle\mathfrak{p}_{i}\right\rangle /\left\langle\mathfrak{p}_{i}^{p}\right\rangle\right) \times \prod_{j=1}^{n}\left(\left\langle\mathfrak{q}_{j}\right\rangle /\left\langle\mathfrak{q}_{j}^{p}\right\rangle\right) \simeq \mathbb{F}_{p}^{s+n}, \quad \operatorname{dim}_{\mathbb{F}_{p}}\left(\mathcal{I}_{L / F} / \mathcal{I}_{F}\right)=s+n, \\
\operatorname{ker}\left(N_{N / L}\right) & \simeq \prod_{i=1}^{s}\left(\left\langle\mathfrak{P}_{i}\left(\mathfrak{P}_{i}^{\prime}\right)^{p-1}\right\rangle /\left\langle\left(\mathfrak{P}_{i}\left(\mathfrak{P}_{i}^{\prime}\right)^{p-1}\right)^{p}\right\rangle\right) \simeq \mathbb{F}_{p}^{s}, \quad \operatorname{dim}_{\mathbb{F}_{p}}\left(\operatorname{ker}\left(N_{N / L}\right)\right)=s .
\end{aligned}
$$

Consequently, the complete space of differential factors has dimension $\operatorname{dim}_{\mathbb{F}_{p}}\left(\mathcal{I}_{N / K} / \mathcal{I}_{K}\right)=n+2 s$.

Proof. Whereas the qualitative formula (4) is valid for any prime $p \geq 2$ and any integer $q>1$ with $\operatorname{gcd}(p, q)=1$, the quantitative description of the norm kernel $\operatorname{ker}\left(N_{N / L}\right)$ is only feasible if we put $q=2$ and therefore have to select an odd prime $p \geq 3$. Replacing $N$ by $L$ and $K$ by $F$ in formula (2), we get $t=n+s$ and thus the first isomorphism of formula (5). For $N$ and $K$, however, we obtain $t=n+2 s$. We point out that, if $s=0$, that is, if none of the totally ramified primes of $L / F$ splits in $N$, then the induced norm mapping $N_{N / L}$ in formula (3) is an isomorphism. For the constitution of the norm kernel, see [33, Theorem 3.4 and Cor. 3.3(3), p. 1994].

\subsection{Primitive ambiguous principal ideals}

The preceding result concerned primitive ambiguous ideals of $N / K$, which can be interpreted as ideal factors of the relative different $\mathfrak{D}_{N / K}$. Formula (2) and Theorem 3.2 show that the $\mathbb{F}_{p}$-dimension of the space $\mathcal{I}_{N / K} / \mathcal{I}_{K}$ increases indefinitely with the number $t$ of totally ramified prime ideals of $N / K$.

Now we restrict our attention to the space $\mathcal{P}_{N / K} / \mathcal{P}_{K}$ of primitive ambiguous principal ideals or differential principal factors (DPF) of $N / K$. We shall see that fundamental constraints from Galois cohomology prohibit an infinite growth of its dimension over $\mathbb{F}_{p}$, for quadratic base fields $K$.

\subsection{Splitting off the capitulation kernel}

We have to cope with a difficulty which arises in the case of a non-trivial class group $\mathrm{Cl}_{K}=\mathcal{I}_{K} / \mathcal{P}_{K}>1$, because then $\mathcal{P}_{N / K} / \mathcal{P}_{K}$ cannot be viewed as a subgroup of $\mathcal{I}_{N / K} / \mathcal{I}_{K}$. Therefore we must separate the capitulation kernel of $N / K$, that is the kernel of the transfer homomorphism $T_{N / K}: \mathrm{Cl}_{K} \rightarrow \mathrm{Cl}_{N}, \mathfrak{a} \cdot \mathcal{P}_{K} \mapsto\left(\mathfrak{a} \mathcal{O}_{N}\right) \cdot \mathcal{P}_{N}$, which extends classes of $K$ to classes of $N$ :

$$
\operatorname{ker}\left(T_{N / K}\right)=\left\{\mathfrak{a} \cdot \mathcal{P}_{K} \mid(\exists A \in N) \mathfrak{a} \mathcal{O}_{N}=A \mathcal{O}_{N}\right\}=\left(\mathcal{I}_{K} \cap \mathcal{P}_{N}\right) / \mathcal{P}_{K}
$$

On the one hand, $\operatorname{ker}\left(T_{N / K}\right)=\left(\mathcal{I}_{K} \cap \mathcal{P}_{N}\right) / \mathcal{P}_{K}$ is a subgroup of $\mathcal{I}_{K} / \mathcal{P}_{K}=\mathrm{Cl}_{K}$, consisting of capitulating ideal classes of $K$. On the other hand, since $\mathcal{I}_{K} \leq \mathcal{I}_{N / K}$ consists of ambiguous ideals of $N / K, \operatorname{ker}\left(T_{N / K}\right)=\left(\mathcal{I}_{K} \cap \mathcal{P}_{N}\right) / \mathcal{P}_{K}$ is a subgroup of $\mathcal{P}_{N / K} / \mathcal{P}_{K}$, consisting of special primitive ambiguous principal ideals of $N / K$, and we can form the quotient

$$
\left(\mathcal{P}_{N / K} / \mathcal{P}_{K}\right) /\left(\left(\mathcal{I}_{K} \cap \mathcal{P}_{N}\right) / \mathcal{P}_{K}\right) \simeq \mathcal{P}_{N / K} /\left(\mathcal{I}_{K} \cap \mathcal{P}_{N}\right)=\mathcal{P}_{N / K} /\left(\mathcal{I}_{K} \cap \mathcal{P}_{N / K}\right) \simeq\left(\mathcal{P}_{N / K} \cdot \mathcal{I}_{K}\right) / \mathcal{I}_{K}
$$

This quotient relation of $\mathbb{F}_{p}$-vector spaces is equivalent to a direct product relation

$$
\mathcal{P}_{N / K} / \mathcal{P}_{K} \simeq\left(\mathcal{P}_{N / K} \cdot \mathcal{I}_{K}\right) / \mathcal{I}_{K} \times \operatorname{ker}\left(T_{N / K}\right)
$$

Since $\left(\mathcal{P}_{N / K} \cdot \mathcal{I}_{K}\right) / \mathcal{I}_{K} \leq \mathcal{I}_{N / K} / \mathcal{I}_{K}$ is an actual inclusion, the factorization of $\mathcal{I}_{N / K} / \mathcal{I}_{K}$ in formula (4) restricts to a factorization

$$
\left(\mathcal{P}_{N / K} \cdot \mathcal{I}_{K}\right) / \mathcal{I}_{K} \simeq\left(\mathcal{P}_{L / F} / \mathcal{P}_{F}\right) \times\left(\operatorname{ker}\left(N_{N / L}\right) \cap\left(\left(\mathcal{P}_{N / K} \cdot \mathcal{I}_{K}\right) / \mathcal{I}_{K}\right)\right),
$$

provided that $F$ is a field with trivial class group $\mathrm{Cl}_{F}$, that is $\mathcal{I}_{F}=\mathcal{P}_{F}$ and thus $\mathcal{P}_{L / F} / \mathcal{P}_{F} \leq \mathcal{I}_{L / F} / \mathcal{I}_{F}$. Combining the formulas (8) and (9) for the rational base field $F=\mathbb{Q}$, we obtain:

Theorem 3.3. There is a trichotomic decomposition of the space $\mathcal{P}_{N / K} / \mathcal{P}_{K}$ of differential principal factors of $N / K$ into three components,

$$
\mathcal{P}_{N / K} / \mathcal{P}_{K} \simeq \mathcal{P}_{L / \mathbb{Q}} / \mathcal{P}_{\mathbb{Q}} \times\left(\operatorname{ker}\left(N_{N / L}\right) \cap\left(\left(\mathcal{P}_{N / K} \mathcal{I}_{K}\right) / \mathcal{I}_{K}\right)\right) \times \operatorname{ker}\left(T_{N / K}\right),
$$

- the absolute principal factors, $\mathcal{P}_{L / \mathbb{Q}} / \mathcal{P}_{\mathbb{Q}}$, of $L / \mathbb{Q}$,

- the relative principal factors, $\operatorname{ker}\left(N_{N / L}\right) \cap\left(\left(\mathcal{P}_{N / K} \mathcal{I}_{K}\right) / \mathcal{I}_{K}\right)$, of $N / K$, and

- the capitulation kernel, $\operatorname{ker}\left(T_{N / K}\right)$, of $N / K$. 


\subsection{Galois cohomology}

In order to establish a quantitative version of the qualitative formula (10), we suppose that $N / K$ is a cyclic relative extension of odd prime degree $p$ and we use the Galois cohomology of the unit group $U_{N}$ as a module over the automorphism group $G=\operatorname{Gal}(N / K)=\langle\sigma\rangle \simeq C_{p}$. In fact, we combine a theorem of Iwasawa [20] on the first cohomology $\mathrm{H}^{1}\left(G, U_{N}\right)$ with a theorem of Hasse [13] on the Herbrand quotient of $U_{N}$ [18], and we use Dirichlet's theorem on the torsion-free unit rank of $K$. By $E_{N / K}=U_{N} \cap \operatorname{ker}\left(N_{N / K}\right)$ we denote the group of relative units of $N / K$.

$$
\begin{aligned}
\mathrm{H}^{1}\left(G, U_{N}\right) & \simeq E_{N / K} / U_{N}^{\sigma-1} \simeq \mathcal{P}_{N / K} / \mathcal{P}_{K} \text { (Iwasawa), } \\
\hat{\mathrm{H}}^{0}\left(G, U_{N}\right) & \simeq U_{K} / N_{N / K}\left(U_{N}\right), \text { with }\left(U_{K}: N_{N / K}\left(U_{N}\right)\right)=p^{U}, 0 \leq U \leq r_{1}+r_{2}-\theta, \\
\frac{\# \mathrm{H}^{1}\left(G, U_{N}\right)}{\# \hat{\mathrm{H}}^{0}\left(G, U_{N}\right)} & =[N: K]=p \quad \text { (Hasse), }
\end{aligned}
$$

where $\left(r_{1}, r_{2}\right)$ is the signature of $K$, and $\theta=0$ if $K$ contains the $p$ th roots of unity, but $\theta=1$ else.

Corollary 3.1. If $N / K$ is cyclic of odd prime degree $p \geq 3$, then the $\mathbb{F}_{p}$-dimensions of the spaces of differential principal factors in Theorem 3.3 are connected by the fundamental equation

$$
U+1=A+R+C, \text { where }
$$

- $A:=\operatorname{dim}_{\mathbb{F}_{p}}\left(\mathcal{P}_{L / \mathbb{Q}} / \mathcal{P}_{\mathbb{Q}}\right)$ is the dimension of absolute principal factors,

- $R:=\operatorname{dim}_{\mathbb{F}_{p}}\left(\operatorname{ker}\left(N_{N / L}\right) \cap\left(\left(\mathcal{P}_{N / K} \mathcal{I}_{K}\right) / \mathcal{I}_{K}\right)\right)$ is the dimension of relative principal factors, and

- $C:=\operatorname{dim}_{\mathbb{F}_{p}}\left(\operatorname{ker}\left(T_{N / K}\right)\right)$ is the dimension of the capitulation kernel.

Corollary 3.2. Under the assumptions $p \geq 3, q=2$ of Theorem 3.1, in particular for $N$ dihedral of degree $2 p$, the dimensions in Corollary 3.1 are bounded by the following fundamental estimates

$$
0 \leq A \leq \min (n+s, m), \quad 0 \leq R \leq \min (s, m), \quad 0 \leq C \leq \min \left(\varrho_{p}, m\right),
$$

where $\varrho_{p}:=\operatorname{rank}_{p}\left(\mathrm{Cl}_{K}\right)$, and $m:=1+r_{1}+r_{2}-\theta$ denotes the cohomological maximum of $U+1$. In particular, we have $m=2$ for real quadratic $K$ with $\left(r_{1}, r_{2}\right)=(2,0)$ or $K=\mathbb{Q}(\sqrt{-3})$ if $p=3$, $m=1$ for imaginary quadratic $K$ with $\left(r_{1}, r_{2}\right)=(0,1)$, except for $K=\mathbb{Q}(\sqrt{-3})$ when $p=3$.

Remark 3.1. For $N$ pure metacyclic of degree $(p-1) p$, the space $\mathcal{P}_{L / \mathbb{Q}} / \mathcal{P}_{\mathbb{Q}}$ of absolute principal factors contains the onedimensional subspace $\Delta=\langle\sqrt[p]{D}\rangle$ generated by the radicals, and thus

$$
1 \leq A \leq \min (t, m), 0 \leq R \leq m-1,0 \leq C \leq \min \left(\varrho_{p}, m-1\right),
$$

where $m=\frac{p+1}{2}$ for cyclotomic $K$ with $\left(r_{1}, r_{2}\right)=\left(0, \frac{p-1}{2}\right)$. In particular, there is no capitulation, $C=0$, for a regular prime $p$ with $\varrho_{p}=0$, for instance $p<37$.

Remark 3.2. We mentioned that in general $\mathcal{P}_{N / K} / \mathcal{P}_{K}$ cannot be viewed as a subgroup of $\mathcal{I}_{N / K} / \mathcal{I}_{K}$. In fact, for a dihedral field $N$ which is unramified with conductor $f=1$ over $K$, we have $n=s=0$, consequently $A=R=0$, and $\mathcal{I}_{N / K} / \mathcal{I}_{K} \simeq 0$ is the nullspace, whereas $\mathcal{P}_{N / K} / \mathcal{P}_{K} \simeq \operatorname{ker}\left(T_{N / K}\right)$ is at least one-dimensional, according to Hilbert's Theorem 94 [19], and at most two-dimensional by the estimate $C \leq \min \left(\varrho_{p}, m\right) \leq \min \left(\varrho_{p}, 2\right) \leq 2$.

In the next two sections, we apply the results of Sections $3.1-3.5$ to various extensions $N / K$.

\subsection{Differential principal factorization (DPF) types of complex dihedral fields}

Let $p$ be an odd prime. We recall the classification theorem for pure cubic fields $L=\mathbb{Q}(\sqrt[3]{D})$ and their Galois closure $N=\mathbb{Q}\left(\zeta_{3}, \sqrt[3]{D}\right)$, that is the metacyclic case $p=3$. The coarse classification of $N$ according to the cohomological invariants $U$ and $A$ alone is closely related to the classification of simply real dihedral fields of degree $2 p$ with any odd prime $p$ by Nicole Moser [36, Dfn. III.1 and Prop. III.3, p. 61], as illustrated in Figure 3. The coarse types $\alpha$ and $\beta$ are completely analogous in both cases. The additional type $\gamma$ is required for pure cubic fields, because there arises the possibility that the primitive cube root of unity $\zeta_{3}$ occurs as relative norm $N_{N / K}(Z)$ of a unit $Z \in U_{N}$. Due to the existence of radicals in the pure cubic case, the $\mathbb{F}_{p}$-dimension $A$ of the vector space of absolute DPF exceeds the corresponding dimension for simply real dihedral fields by one.

The fine classification of $N$ according to the invariants $U, A, R$ and $C$ in the simply real dihedral situation with $U+1=$ $A+R+C$ splits type $\alpha$ with $A=0$ further in type $\alpha_{1}$ with $C=1$ (capitulation) and type $\alpha_{2}$ with $R=1$ (relative DPF). In the pure cubic situation, however, no further splitting occurs, since $C=0$, and $R=U+1-A$ is determined uniquely by $U$ and $A$ already. We oppose the two classifications in the following theorems. 

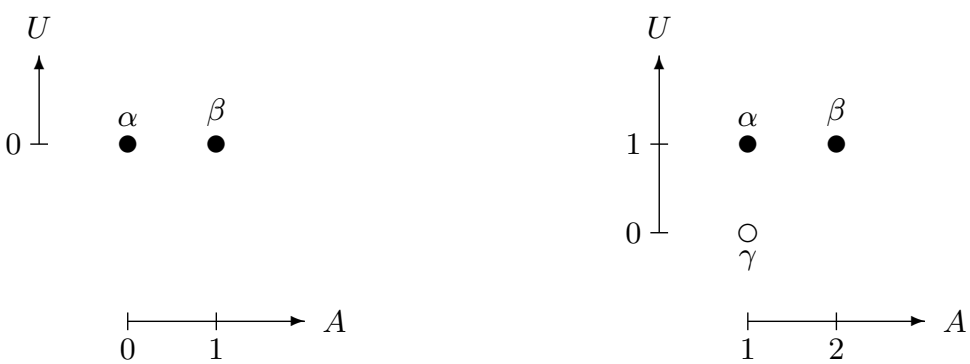

Figure 3: Classification of simply real dihedral and pure cubic fields.

Theorem 3.4. Each simply real dihedral field $N / \mathbb{Q}$ of absolute degree $[N: \mathbb{Q}]=2 p$ with an odd prime $p$ belongs to precisely one of the following 3 differential principal factorization types, in dependence on the triplet $(A, R, C)$ :

\begin{tabular}{|c||c||c||ccc|}
\hline Type & $U$ & $U+1=A+R+C$ & $A$ & $R$ & $C$ \\
\hline$\alpha_{1}$ & 0 & 1 & 0 & 0 & 1 \\
$\alpha_{2}$ & 0 & 1 & 0 & 1 & 0 \\
$\beta$ & 0 & 1 & 1 & 0 & 0 \\
\hline
\end{tabular}

Proof. Consequence of Cor. 3.1 and 3.2. See [36, Dfn. III.1 and Prop. III.3, p. 61] and [26].

Theorem 3.5. Each pure metacyclic field $N=\mathbb{Q}\left(\zeta_{3}, \sqrt[3]{D}\right)$ of absolute degree $[N: \mathbb{Q}]=6$ with cube free radicand $D \in \mathbb{Z}$, $D \geq 2$, belongs to precisely one of the following 3 differential principal factorization types, in dependence on the invariant $U$ and the pair $(A, R)$ :

\begin{tabular}{|c||c||c||cc|}
\hline Type & $U$ & $U+1=A+R$ & $A$ & $R$ \\
\hline$\alpha$ & 1 & 2 & 1 & 1 \\
$\beta$ & 1 & 2 & 2 & 0 \\
\hline$\gamma$ & 0 & 1 & 1 & 0 \\
\hline
\end{tabular}

Proof. A part of the proof is due to Barrucand and Cohn [4] who distinguished 4 different types, I스 $\beta$, II, III $\hat{=} \alpha$, and IV $\hat{=} \gamma$. However, Halter-Koch [12] showed the impossibility of one of these types, namely type II. Our new proof with different methods is given in [3, Theorem 2.1, p. 254].

\subsection{Differential principal factorization (DPF) types of real dihedral fields}

Now we state the classification theorem for pure quintic fields $L=\mathbb{Q}(\sqrt[5]{D})$ and their Galois closure $N=\mathbb{Q}\left(\zeta_{5}, \sqrt[5]{D}\right)$, that is the metacyclic case $p=5$. The coarse classification of $N$ according to the invariants $U$ and $A$ alone is closely related to the classification of totally real dihedral fields of degree $2 p$ with any odd prime $p$ by Nicole Moser [36, Theorem III.5, p. 62], as illustrated in Figure 4. The coarse types $\alpha, \beta, \gamma, \delta, \varepsilon$ are completely analogous in both cases. Additional types $\zeta, \eta, \vartheta$ are required for pure quintic fields, because there arises the possibility that the primitive fifth root of unity $\zeta_{5}$ occurs as relative norm $N_{N / K}(Z)$ of a unit $Z \in U_{N}$. Due to the existence of radicals in the pure quintic case, the $\mathbb{F}_{p}$-dimension $A$ of the vector space of absolute DPF exceeds the corresponding dimension for totally real dihedral fields by one (see Remark 3.1).

The fine classification of $N$ according to the invariants $U, A, R$ and $C$ in the totally real dihedral situation with $U+1=$ $A+R+C$ splits type $\alpha$ with $U=1, A=0$ further in type $\alpha_{1}$ with $C=2$ (double capitulation), type $\alpha_{2}$ with $C=R=1$ (mixed capitulation and relative DPF), type $\alpha_{3}$ with $R=2$ (double relative DPF), type $\beta$ with $U=A=1$ in type $\beta_{1}$ with $C=1$ (capitulation), type $\beta_{2}$ with $R=1$ (relative DPF), and type $\delta$ with $U=A=0$ in type $\delta_{1}$ with $C=1$ (capitulation), type $\delta_{2}$ with $R=1$ (relative DPF). In the pure quintic situation with $U+1=A+I+R$ [33], however, we arrive at the second of the following theorems where we oppose the two classifications.

Theorem 3.6. Each totally real dihedral field $N / \mathbb{Q}$ of absolute degree $[N: \mathbb{Q}]=2 p$ with an odd prime $p$ belongs to precisely one of the following 9 differential principal factorization types, in dependence on the invariant $U$ and the triplet $(A, R, C)$. 

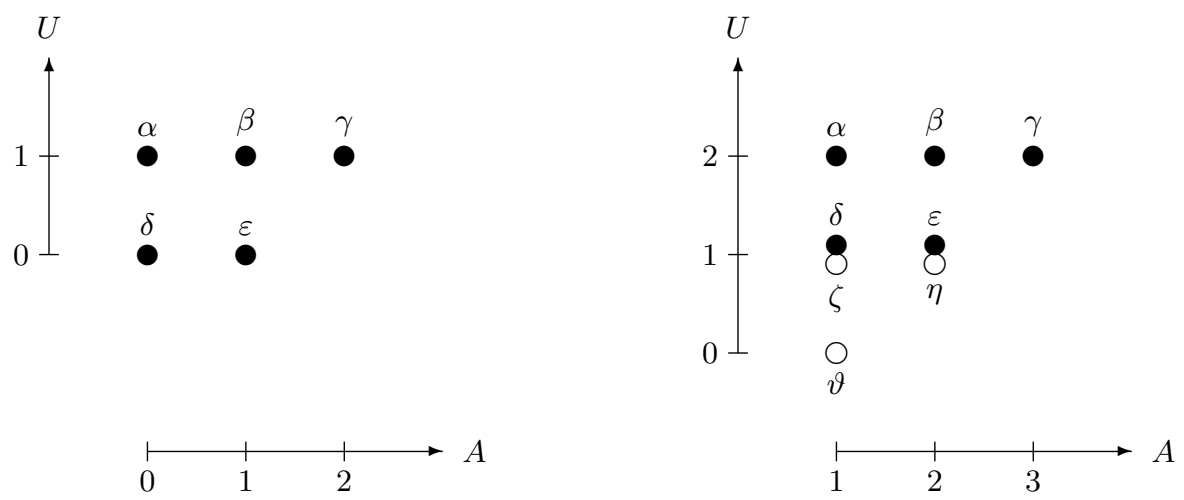

Figure 4: Classification of totally real dihedral and pure quintic fields.

\begin{tabular}{|c||c||c||ccc|}
\hline Type & $U$ & $U+1=A+R+C$ & $A$ & $R$ & $C$ \\
\hline$\alpha_{1}$ & 1 & 2 & 0 & 0 & 2 \\
$\alpha_{2}$ & 1 & 2 & 0 & 1 & 1 \\
$\alpha_{3}$ & 1 & 2 & 0 & 2 & 0 \\
$\beta_{1}$ & 1 & 2 & 1 & 0 & 1 \\
$\beta_{2}$ & 1 & 2 & 1 & 1 & 0 \\
$\gamma$ & 1 & 2 & 2 & 0 & 0 \\
\hline$\delta_{1}$ & 0 & 1 & 0 & 0 & 1 \\
$\delta_{2}$ & 0 & 1 & 0 & 1 & 0 \\
$\varepsilon$ & 0 & 1 & 1 & 0 & 0 \\
\hline
\end{tabular}

Proof. Consequence of the Corollaries 3.1 and 3.2. See also [36, Theorem III.5, p. 62] and [26].

Theorem 3.7. Each pure metacyclic field $N=\mathbb{Q}\left(\zeta_{5}, \sqrt[5]{D}\right)$ of absolute degree $[N: \mathbb{Q}]=20$ with 5-th power free radicand $D \in \mathbb{Z}, D \geq 2$, belongs to precisely one of the following 13 differential principal factorization types, in dependence on the invariant $U$ and the triplet $(A, I, R)$.

\begin{tabular}{|c||c||c||ccc|}
\hline Type & $U$ & $U+1=A+I+R$ & $A$ & $I$ & $R$ \\
\hline$\alpha_{1}$ & 2 & 3 & 1 & 0 & 2 \\
$\alpha_{2}$ & 2 & 3 & 1 & 1 & 1 \\
$\alpha_{3}$ & 2 & 3 & 1 & 2 & 0 \\
$\beta_{1}$ & 2 & 3 & 2 & 0 & 1 \\
$\beta_{2}$ & 2 & 3 & 2 & 1 & 0 \\
$\gamma$ & 2 & 3 & 3 & 0 & 0 \\
\hline$\delta_{1}$ & 1 & 2 & 1 & 0 & 1 \\
$\delta_{2}$ & 1 & 2 & 1 & 1 & 0 \\
$\varepsilon$ & 1 & 2 & 2 & 0 & 0 \\
\hline$\zeta_{1}$ & 1 & 2 & 1 & 0 & 1 \\
$\zeta_{2}$ & 1 & 2 & 1 & 1 & 0 \\
$\eta$ & 1 & 2 & 2 & 0 & 0 \\
\hline$\vartheta$ & 0 & 1 & 1 & 0 & 0 \\
\hline
\end{tabular}

The types $\delta_{1}, \delta_{2}$, $\varepsilon$ are characterized additionally by $\zeta_{5} \notin N_{N / K}\left(U_{N}\right)$, and the types $\zeta_{1}, \zeta_{2}, \eta$ by $\zeta_{5} \in N_{N / K}\left(U_{N}\right)$.

Proof. The proof is given in [33, Theorem 6.1].

Remark 3.3. Our classification of totally real dihedral fields in Theorem 3.6 refines the classification by Moser [36] who uses the results on integral representations of the dihedral group $D_{p}$ by Lee [21]. She denotes by $b=\left(U_{K}: N_{N / K}\left(U_{N}\right)\right)$ the unit norm index and obtains $U_{N}=U_{K} \cdot E_{N / K}$ as a split extension (direct product) of $U_{K}$ by $E_{N / K}$ for $b=p$ (types $\alpha, \beta, \gamma$ ), and $\left(U_{N}: U_{K} \cdot E_{N / K}\right)=p$ as a non-split extension (of modules over $\mathbb{Z}\left[D_{p}\right]$ ) for $b=1$ (types $\delta, \varepsilon$ ), due to a non-trivial relation $N_{N / K}(H)=H^{1+\sigma+\ldots+\sigma^{p-1}}=\eta$ for the fundamental unit $\eta$ of $K$ and $a$ unit $H \in U_{N} \backslash\left(U_{K} \cdot E_{N / K}\right)$. 
For $p=3$, a geometric interpretation of the unit lattice in logarithmic space, i.e., the Dirichlet-Minkowski image of $U_{N}$, has been given by Hasse [15, 16].

\section{Classifying Angell's range $0<d_{L}<100000$}

In Table 1 and all the following tables, we present the results of our classification of totally real cubic fields $L$ and their normal closures $N$ into differential principal factorization types $\tau(L)=\tau(N)$. The rows correspond to the numerous steps where we applied our algorithm (Section 2.3) to various configurations of 3-class rank $\varrho_{3}$ of the real quadratic subfield $K$ of $N$ and 3-admissible conductors $f$ of $N / K$. Here, $d$ denotes the fundamental discriminant of $K$, and $q, q_{1}, q_{2}$, respectively $\ell, \ell_{1}, \ell_{2}$, denote prime numbers congruent to 2 , respectively 1 modulo 3 . In Table 1 , the types $\alpha_{2}$ and $\alpha_{3}$ do not yet occur.

Table 1: Totally real cubic discriminants $d_{L}=f^{2} \cdot d$ in the range $0<d_{L}<10^{5}$.

\begin{tabular}{|c|c|c|c|c|c|c|c|c|c|c|c|c|c|c|}
\hline \multirow[b]{2}{*}{$f$} & \multirow[b]{2}{*}{ Condition } & \multicolumn{5}{|c|}{ Multiplicity } & \multicolumn{7}{|c|}{ Differential Principal Factorization } & \multirow[b]{2}{*}{ Total } \\
\hline & & 0 & 1 & 2 & 3 & 4 & $\alpha_{1}$ & $\beta_{1}$ & $\beta_{2}$ & $\gamma$ & $\delta_{1}$ & $\delta_{2}$ & $\varepsilon$ & \\
\hline 1 & $\varrho_{3}=0$ & 27089 & & & & & & & & & & & & 0 \\
\hline$q$ & $\equiv 2(\bmod 3)$ & 2219 & 806 & & & & & & & & & & 806 & 806 \\
\hline 3 & $d \equiv 3(\bmod 9)$ & 287 & 109 & & & & & & & & & & 109 & 109 \\
\hline 3 & $d \equiv 6(\bmod 9)$ & 284 & 105 & & & & & & & & & & 105 & 105 \\
\hline 9 & $d \equiv 6(\bmod 9)$ & 9 & 38 & & 1 & & & & & & & & 41 & 41 \\
\hline 9 & $d \equiv 2(\bmod 3)$ & 102 & 34 & & & & & & & & & & 34 & 34 \\
\hline 9 & $d \equiv 1(\bmod 3)$ & 96 & 31 & & & & & & 8 & & & 20 & 3 & 31 \\
\hline$\ell$ & $\equiv 1(\bmod 3)$ & 316 & 86 & & & & & & 20 & & & 59 & 7 & 86 \\
\hline$q_{1} q_{2}$ & & 30 & 38 & 2 & & & & & & 38 & & & 4 & $\overline{42}$ \\
\hline $3 q$ & $d \equiv 3(\bmod 9)$ & 23 & 23 & & & & & & & 23 & & & & 23 \\
\hline $3 q$ & $d \equiv 6(\bmod 9)$ & 19 & 25 & 1 & & & & & & 25 & & & 2 & 27 \\
\hline $9 q$ & $d \equiv 6(\bmod 9)$ & & & 4 & 1 & & & & & 9 & & & 2 & 11 \\
\hline $9 q$ & $d \equiv 2(\bmod 3)$ & 6 & 8 & & & & & & & 8 & & & & 8 \\
\hline $9 q$ & $d \equiv 1(\bmod 3)$ & 5 & 10 & & & & & & 10 & & & & & 10 \\
\hline$q \ell$ & & 13 & 29 & 1 & & & & & 29 & & & & 2 & 31 \\
\hline $3 \ell$ & $d \equiv 3(\bmod 9)$ & 1 & 5 & & & & & & 5 & & & & & 5 \\
\hline $3 \ell$ & $d \equiv 6(\bmod 9)$ & 2 & 3 & & & & & & 3 & & & & & 3 \\
\hline $9 \ell$ & $d \equiv 2(\bmod 3)$ & & 1 & & & & & & 1 & & & & & 1 \\
\hline $3 q_{1} q_{2}$ & $d \equiv 3(\bmod 9)$ & & 1 & 1 & & & & & & 3 & & & & 3 \\
\hline 1 & $\varrho_{3}=1$ & & 3300 & & & & & & & & 3300 & & & 3300 \\
\hline$q$ & $\equiv 2(\bmod 3)$ & 261 & & & 14 & & & 4 & & & 36 & & 2 & 42 \\
\hline 3 & $d \equiv 3(\bmod 9)$ & 27 & & & & & & & & & & & & 0 \\
\hline 3 & $d \equiv 6(\bmod 9)$ & 34 & & & 1 & & & & & & 3 & & & 3 \\
\hline 9 & $d \equiv 6(\bmod 9)$ & 1 & & & & & & & & & & & & 0 \\
\hline 9 & $d \equiv 2(\bmod 3)$ & 6 & & & & & & & & & & & & 0 \\
\hline 9 & $d \equiv 1(\bmod 3)$ & 10 & & & & & & & & & & & & 0 \\
\hline$\ell$ & $\equiv 1(\bmod 3)$ & 25 & & & 3 & & & 3 & & & 6 & & & 9 \\
\hline$q_{1} q_{2}$ & & 1 & & & & & & & & & & & & 0 \\
\hline $3 q$ & $d \equiv 3(\bmod 9)$ & 1 & & & & & & & & & & & & 0 \\
\hline $3 q$ & $d \equiv 6(\bmod 9)$ & 1 & & & 1 & & & 3 & & & & & & 3 \\
\hline $9 q$ & $d \equiv 1(\bmod 3)$ & 1 & & & & & & & & & & & & 0 \\
\hline$\overline{1} 1$ & $\varrho_{3}=2$ & & & & & 5 & 16 & & & & 4 & & & 20 \\
\hline & Summary & & 4652 & 9 & 21 & $\overline{5}$ & $\overline{16}$ & 10 & 76 & 106 & 3349 & 79 & 1117 & $4 \mathbf{4 7 5 3}$ \\
\hline
\end{tabular}

According to Table 1, the number of non-cyclic totally real cubic fields $L$ with discriminant $0<d_{L}<10^{5}$ is $\mathbf{4 7 5 3}$, in perfect accordance with the results by Llorente and Oneto [22,23], who discovered the ommission of ten fields in the table by Angell [1,2]. Together with 51 cyclic cubic fields in Table 2, the total number is $\mathbf{4 8 0 4}$ (rather than 4794, as announced erroneously in [2]).

According to Table 2, the number of cyclic cubic fields $L$ with discriminant $0<d_{L}<10^{5}$ is $\mathbf{5 1}$, with 31 arising from singlets having conductors $f$ with a single prime divisor, and 20 from doublets having two prime divisors of the conductor $f$. (M denotes the multiplicity.) 
Table 2: Cyclic cubic discriminants $d_{L}=f^{2}$ in the range $0<d_{L}<10^{5}$.

\begin{tabular}{|rl||rr||rr||rr|}
\hline \multicolumn{1}{|c||}{} & \multicolumn{2}{c|}{ M } & DPF & $f$ & $d_{L}$ \\
\hline 9 & $d=1$ & 1 & 2 & $\zeta$ & & \\
$\ell$ & $\equiv+1(\bmod 3)$ & 30 & & 1 & 9 & 81 \\
\hline $9 \ell$ & $d=1$ & & 4 & 8 & 7 & 49 \\
$\ell_{1} \ell_{2}$ & $\equiv+1(\bmod 3)$ & & 6 & 12 & 91 & 8281 \\
\hline & Summary & 31 & 10 & 51 & & \\
\hline
\end{tabular}

Although we have given a succinct survey of the DPF types of all multiplets in Angell's range $0<d_{L}<10^{5}$ in the conclusion of [35], we arrange them again in a more ostensive tabular form with absolute frequency and minimal discriminant $d_{L}=f^{2} \cdot d$.

All doublets in Table 3 are pure. In bigger ranges, there will also occur mixed doublets, e.g. in Table 19. The corresponding 3 -class rank is always $\varrho=0$.

Table 3: Types of doublets in the range $0<d_{L}<10^{5}$.

\begin{tabular}{|c||r||rrr|}
\hline DPF Type & Frequency & $d$ & $f$ & $d_{L}$ \\
$\left(\tau\left(L_{1}\right), \tau\left(L_{2}\right)\right)$ & & & & \\
\hline$(\gamma, \gamma)$ & 4 & 33 & 45 & 66825 \\
$(\varepsilon, \varepsilon)$ & 5 & 373 & 10 & 37300 \\
\hline Total: & 9 & & & \\
\hline
\end{tabular}

The triplets with $\varrho=1$ in Table 4 have been partially classified in a coarse sense by Schmithals in 1985 [38]. He merely decided whether capitulation occurs or not, indicating $C=1$ by the symbol " + " and $C=0$ by "-". This admits the detection of type $\varepsilon$ but fails to distinguish between the types $\beta_{1}$ and $\delta_{1}$.

Table 4: Types of triplets in the range $0<d_{L}<10^{5}$.

\begin{tabular}{|c|c||r||rrr|}
\hline & $\begin{array}{c}\text { DPF Type } \\
\varrho\end{array}\left(\tau\left(L_{1}\right), \ldots, \tau\left(L_{3}\right)\right)$ & Frequency & $d$ & $f$ & $d_{L}$ \\
\hline 0 & $(\gamma, \gamma, \gamma)$ & 1 & 69 & 18 & 22356 \\
0 & $(\varepsilon, \varepsilon, \varepsilon)$ & 1 & 717 & 9 & 58077 \\
\hline 1 & $\left(\beta_{1}, \beta_{1}, \beta_{1}\right)$ & 1 & 1509 & 6 & 54324 \\
1 & $\left(\beta_{1}, \beta_{1}, \varepsilon\right)$ & 2 & 14397 & 2 & 57588 \\
1 & $\left(\beta_{1}, \delta_{1}, \delta_{1}\right)$ & 3 & 1765 & 7 & 86485 \\
1 & $\left(\delta_{1}, \delta_{1}, \delta_{1}\right)$ & 13 & 7053 & 2 & 28212 \\
\hline & Total: & 21 & & & \\
\hline
\end{tabular}

The quartets in Table 5 belong to unramified cyclic cubic extensions of quadratic fields with $\varrho=2$. In fact, they have been classified by Heider and Schmithals in 1982 [17, p. 24]. In 2006, respectively 2008, respectively 2009, we have detected the remaining capitulation numbers $\nu(K)=0$, respectively 1 , respectively 2 , which show up in Table 16. See [29,32].

\subsection{Numerical results by Nicole Moser}

In her paper [36] on the units $U_{N}$ and class groups $\mathrm{Cl}_{N}$ of dihedral fields $N$ of degree $2 p$ with an odd prime $p$, Nicole Moser has given a small table [36, V.4, pp. 72-73] of 34 totally real cubic fields $L$ with discriminants $0<d_{L}<1500$ in order to illustrate her (coarse) classification by concrete examples for $p=3$. She found 26 fields of type $\delta$, unramified with conductor $f=1$, without exceptions, and thus more precisely of our finer type $\delta_{1}$. The frequency $\frac{26}{34} \approx 76 \%$ corresponds to Angell's $\frac{3349}{4753} \approx 70 \%$. Discriminants $d_{L}=1^{2} \cdot d=d$ are 
Table 5: Types of quartets in the range $0<d_{L}<10^{5}$.

\begin{tabular}{|c||c|r||c|}
\hline $\begin{array}{c}\text { DPF Type } \\
\left(\tau\left(L_{1}\right), \ldots, \tau\left(L_{4}\right)\right)\end{array}$ & $\begin{array}{c}\text { Capitulation Number } \nu(K) \\
\text { (according to [8]) }\end{array}$ & Frequency & $d_{L}=d$ \\
\hline$\left(\alpha_{1}, \alpha_{1}, \alpha_{1}, \alpha_{1}\right)$ & 4 & 1 & 62501 \\
$\left(\alpha_{1}, \alpha_{1}, \alpha_{1}, \delta_{1}\right)$ & 3 & 4 & 32009 \\
\hline & Total: & 5 & \\
\hline
\end{tabular}

In fact, each of the normal closures $N$ of degree 3 over its quadratic subfield $K$ is precisely the Hilbert 3-class field $\mathrm{F}_{3}^{1}(K)$ of $K$, with one exception $d=\mathbf{1 1 2 9}$, where $K$ has class number 9. Here, Moser's table entries $a=9, h_{N}=9$ are incorrect and must be replaced by $a=3, h_{N}=3$. She uses two invariants, the index of subfield units $a=\left(U_{N}:\left(U_{K} \cdot U_{L} \cdot U_{L^{\sigma}}\right)\right)$ in Formula (16), and the unit norm index $b=\left(U_{K}: N_{N / K}\left(U_{N}\right)\right)$ in Remark 3.3 for her characterization of the types $\alpha, \beta, \gamma, \delta, \varepsilon$.

Among the remaining 8 fields, one is of type $\gamma$ with $d=21 \equiv 3(\bmod 9), f=2 \cdot 3, d_{L}=6^{2} \cdot 21=756$, and seven are of type $\varepsilon$. Among the latter, five have $f=2$ and $d \in\{37,101,141,197,269\}, d_{L} \in\{148,404,564,788,1076\}$, two have $f=3$ and $d=69 \equiv 6(\bmod 9), d_{L}=621$, respectively $d=93 \equiv 3(\bmod 9), d_{L}=837$. The frequency $\frac{7}{34} \approx 21 \%$ corresponds to Angell's $\frac{1117}{4753} \approx 24 \%$.

However, it must be pointed out that 4 fields are missing: one is of type $\gamma$ with $d=13, f=2 \cdot 5, d_{L}=10^{2} \cdot 13=1300$, and three are of type $\varepsilon$ with $d=349, f=2, d_{L}=1396$, respectively $d=57, f=5, d_{L}=1425$, respectively $d=373, f=2$, $d_{L}=1492$.

On the other hand, it is very instructive that there is also a superfluous field: although $f=2$ is a 3-admissible conductor for $d=229$, since 2 remains inert in $K=\mathbb{Q}(\sqrt{d}), D=2^{2} \cdot d=4 \cdot 229=916$ is only a formal cubic discriminant, because the defect of 2 is $\delta(2)=1$. So $\mathbf{M}_{4 d}=\emptyset$ is a nilet, and the given polynomial $X^{3}-X^{2}-6 X+4$ generates the cubic field with conductor $f=1$ and discriminant $d_{L}=229$. In this case, Moser is uncertain whether the type of the hypothetical cubic field with discriminant 916 is $\varepsilon$ or $\gamma$. Type $\gamma$, however, is never possible for a field with prime conductor, such as $f=2$. Since $\varrho=1$ for $d=229$, the types $\beta_{1}, \delta_{1}$ and $\varepsilon$ would be possible, but Moser's claim $a=9$ discourages types $\beta_{1}$ and $\delta_{1}$. It is mysterious how she determined the invariant $a$ for a non-existent field without knowing the class number $h_{N}$. In view of the errors in Moser's table, it is worth ones while to state a summarizing theorem which also pays attention to the modest contribution by cyclic cubic fields. Cutting off Table 1 at $d_{L}=1500$ we obtain:

Theorem 4.1. Among the 44 totally real cubic fields L with discriminants $0<d_{L}<1500$, there are 2 (4\%) of type $\gamma$, 26 (59\%) of type $\delta_{1}$, and 10 (23\%) of type $\varepsilon$. These 38 non-Galois cubic fields are complemented by 6 (14\%) cyclic cubic fields with conductors $f \in\{7,9,13,19,31,37\}$. With respect to the multiplicity $m$, all 44 fields form singlets with $m=1$.

Obviously, Moser was not in possession of Angell's UMT [1], otherwise she would have been able to detect the gaps in her table. She rather refers to an unpublished table by René Smadja.

Outside of the range $0<d_{L}<1500$, Moser gives an example of a field with type $\beta$, more precisely our finer type $\beta_{2}$, for $d=29, f=2 \cdot 7, d_{L}=14^{2} \cdot 29=5684$. The conductor is divisible by the prime 7 which splits in $K$, as required for type $\beta_{2}$.

Moser did not know any examples of her type $\alpha$. From Table 5 we know that the minimal discriminant of such a field is 32009 , discovered by Heider and Schmithals [17]. Due to $f=1$ it is more precisely our finer type $\alpha_{1}$. See Theorem 8.2 and Example 8.4.

\section{Update of our 1991 classification for $0<d_{L}<200000$}

As announced in [35], Table 1 with $0<d_{L}<10^{5}$ was completed on Tuesday, 29 December 2020. One week later, on Tuesday, 05 January 2021, we finished the updated Table 6 containing the revised classification of all totally real cubic fields $L$ with discriminants $0<d_{L}<2 \cdot 10^{5}$, which we had investigated in August 1991 [27].

According to Table 6, the total number of all non-cyclic totally real cubic fields $L$ with discriminants $d<2 \cdot 10^{5}$ is 9945 . Together with 70 cyclic cubic fields in Table 7 the number is $\mathbf{1 0 0 1 5}$,

According to Table 7, the number of cyclic cubic fields $L$ with discriminant $0<d_{L}<2 \cdot 10^{5}$ is $\mathbf{7 0}$, with 42 arising from singlets having conductors $f$ with a single prime divisor, and 28 from doublets having two prime divisors of the conductor f. (M denotes the multiplicity.)

As predicted in the introduction, several fields of type $\beta_{2}$ were unduly classified as type $\delta_{2}$, since Voronoi's algorithm [40] did not find any absolute principal factors along the chains of lattice minima. Since the table [27, p. 3] accumulates 
Table 6: Totally real cubic discriminants $d_{L}=f^{2} \cdot d$ in the range $0<d_{L}<2 \cdot 10^{5}$.

\begin{tabular}{|c|c|c|c|c|c|c|c|c|c|c|c|c|c|c|c|}
\hline \multirow[b]{2}{*}{$f$} & \multirow[b]{2}{*}{ Condition } & \multicolumn{5}{|c|}{ Multiplicity } & \multicolumn{8}{|c|}{ Differential Principal Factorization } & \multirow[b]{2}{*}{ Total } \\
\hline & & 0 & 1 & 2 & 3 & 4 & $\alpha_{1}$ & $\alpha_{3}$ & $\beta_{1}$ & $\beta_{2}$ & $\gamma$ & $\delta_{1}$ & $\delta_{2}$ & $\varepsilon$ & \\
\hline 1 & $\varrho_{3}=0$ & 53848 & & & & & & & & & & & & & 0 \\
\hline$q$ & $\equiv 2(\bmod 3)$ & 4338 & 1656 & & & & & & & & & & & 1656 & 1656 \\
\hline 3 & $d \equiv 3(\bmod 9)$ & 551 & 221 & & & & & & & & & & & 221 & 221 \\
\hline 3 & $d \equiv 6(\bmod 9)$ & 561 & 222 & & & & & & & & & & & 222 & 222 \\
\hline 9 & $d \equiv 6(\bmod 9)$ & 22 & 68 & & 3 & & & & & & & & & 77 & 77 \\
\hline 9 & $d \equiv 2(\bmod 3)$ & 192 & 74 & & & & & & & & & & & 74 & 74 \\
\hline 9 & $d \equiv 1(\bmod 3)$ & 184 & 71 & & & & & & & 17 & & & 48 & 6 & 71 \\
\hline$\ell$ & $\equiv 1(\bmod 3)$ & 624 & 196 & & & & & & & 45 & & & 140 & 11 & 196 \\
\hline$q_{1} q_{2}$ & & 65 & 66 & 2 & & & & & & & 66 & & & 4 & 70 \\
\hline $3 q$ & $d \equiv 3(\bmod 9)$ & 45 & 46 & 1 & & & & & & & 46 & & & 2 & 48 \\
\hline $3 q$ & $d \equiv 6(\bmod 9)$ & 35 & 45 & 6 & & & & & & & 45 & & & 12 & 57 \\
\hline $9 q$ & $d \equiv 6(\bmod 9)$ & & & 8 & 1 & & & & & & 15 & & & 4 & 19 \\
\hline $9 q$ & $d \equiv 2(\bmod 3)$ & 15 & 16 & & & & & & & & 16 & & & & 16 \\
\hline $9 q$ & $d \equiv 1(\bmod 3)$ & 10 & 20 & & & & & & & 20 & & & & & 20 \\
\hline$q \ell$ & & 30 & 57 & 3 & & & & & & 56 & 1 & & & 6 & 63 \\
\hline $3 \ell$ & $d \equiv 3(\bmod 9)$ & 4 & 9 & & & & & & & 9 & & & & & 9 \\
\hline $3 \ell$ & $d \equiv 6(\bmod 9)$ & 6 & 5 & & & & & & & 5 & & & & & 5 \\
\hline $9 \ell$ & $d \equiv 2(\bmod 3)$ & 1 & 3 & & & & & & & 3 & & & & & 3 \\
\hline $9 \ell$ & $d \equiv 1(\bmod 3)$ & & 1 & & & & & 1 & & & & & & & 1 \\
\hline$q_{1} q_{2} q_{3}$ & & & & 1 & & & & & & & 2 & & & & 2 \\
\hline $3 q_{1} q_{2}$ & $d \equiv 3(\bmod 9)$ & & 1 & 1 & & & & & & & 3 & & & & 3 \\
\hline $3 q_{1} q_{2}$ & $d \equiv 6(\bmod 9)$ & & & 1 & & & & & & & 2 & & & & 2 \\
\hline $9 q_{1} q_{2}$ & $d \equiv 1(\bmod 3)$ & & 1 & & & & & & & & 1 & & & & 1 \\
\hline$q_{1} q_{2} \ell$ & & & & 1 & & & & & & & 2 & & & & 2 \\
\hline $3 q \ell$ & $d \equiv 3(\bmod 9)$ & & & 1 & & & & & & & 2 & & & & 2 \\
\hline 1 & 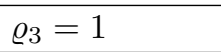 & & 6924 & & & & & & & & & 6924 & & & $\overline{66924}$ \\
\hline$q$ & $\equiv 2(\bmod 3)$ & 576 & & & 28 & & & & 12 & & & 68 & & 4 & 84 \\
\hline 3 & $d \equiv 3(\bmod 9)$ & 66 & & & 2 & & & & 2 & & & 3 & & 1 & 6 \\
\hline 3 & $d \equiv 6(\bmod 9)$ & 63 & & & 2 & & & & & & & 6 & & & 6 \\
\hline 9 & $d \equiv 6(\bmod 9)$ & 3 & & & & & & & & & & & & & 0 \\
\hline 9 & $d \equiv 2(\bmod 3)$ & 16 & & & 1 & & & & & & & 3 & & & 3 \\
\hline 9 & $d \equiv 1(\bmod 3)$ & 20 & & & & & & & & & & & & & 0 \\
\hline$\ell$ & $\equiv 1(\bmod 3)$ & 51 & & & 5 & & & & 4 & & & 10 & & 1 & 15 \\
\hline $\begin{array}{r}q_{1} q_{2} \\
3 q\end{array}$ & $d \equiv 3(\bmod 9)$ & $\begin{array}{l}3 \\
3\end{array}$ & & & & & & & & & & & & & $\begin{array}{l}0 \\
0\end{array}$ \\
\hline $3 q$ & $d \equiv 6(\bmod 9)$ & 4 & & & 1 & & & & 3 & & & & & & 3 \\
\hline $9 q$ & $d \equiv 1(\bmod 3)$ & 2 & & & & & & & & & & & & & 0 \\
\hline 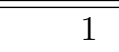 & $\overline{\varrho_{3}=2}$ & & & & & 16 & 50 & & & & & 14 & & & 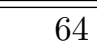 \\
\hline & Summary & & 9702 & 25 & 43 & $\overline{16}$ & 50 & 1 & 21 & 155 & 201 & $\overline{7028}$ & 188 & 2301 & $\overline{9945}$ \\
\hline
\end{tabular}

Table 7: Cyclic cubic discriminants $d_{L}=f^{2}$ in the range $0<d_{L}<2 \cdot 10^{5}$.

\begin{tabular}{|rl||rr||r|}
\hline \multicolumn{1}{|c||}{$f$ Condition } & \multicolumn{2}{c||}{1} & 2 & DPF \\
\hline 9 & $d=1$ & 1 & & 1 \\
$\ell$ & $\equiv 1(\bmod 3)$ & 41 & & 41 \\
\hline $9 \ell$ & $d=1$ & & 6 & 12 \\
$\ell_{1} \ell_{2}$ & $\equiv 1(\bmod 3)$ & & 8 & 16 \\
\hline \multicolumn{2}{|c||}{ Summary } & 42 & 14 & 70 \\
\hline
\end{tabular}


information on conductors with similar behavior and thus has a format different from Table 6 , we compile a translation of critical rows in both tables, subtracting contributions by cyclic cubic fields in [27].

Table 8: Accumulation of rows in Table 6 for comparison with [27, p. 3].

\begin{tabular}{|c|c|c|c|c|c|c|c|c|c|c|c|c|c|c|}
\hline \multirow[b]{2}{*}{$f$} & \multirow[b]{2}{*}{ Condition } & \multicolumn{4}{|c|}{ Multiplicity } & \multicolumn{8}{|c|}{ Differential Principal Factorization } & \multirow[b]{2}{*}{ Total } \\
\hline & & 1 & 2 & 3 & 4 & $\alpha_{1}$ & $\alpha_{3}$ & $\beta_{1}$ & $\beta_{2}$ & $\gamma$ & $\delta_{1}$ & $\delta_{2}$ & $\varepsilon$ & \\
\hline 9 & $d \equiv 1(\bmod 3), \varrho=0$ & 71 & & & & & & & 17 & & & 48 & 6 & 71 \\
\hline$\ell$ & $\equiv 1(\bmod 3), \varrho=0$ & 196 & & & & & & & 45 & & & 140 & 11 & 196 \\
\hline & Together & 267 & & & & & & & 62 & & & 188 & 17 & 267 \\
\hline$\ell$ & $\equiv 1(\bmod 3), \varrho=1$ & & & 5 & & & & 4 & & & 10 & & 1 & 15 \\
\hline & Summary & 9702 & 25 & 43 & 16 & 50 & 1 & 21 & 155 & 201 & 7028 & 188 & 2301 & 9945 \\
\hline [27] & $\bar{m} m=1, t=s=1, \varrho=0$ & 267 & & & & & & & 53 & & & 198 & 16 & 267 \\
\hline [27] & $m=3, t=s=1, \varrho=1$ & & & 5 & & & & 3 & & & 11 & & 1 & 15 \\
\hline [27] & total & 9702 & 25 & 43 & 16 & 50 & 1 & 20 & 146 & 201 & 7029 & 198 & 2300 & 9945 \\
\hline
\end{tabular}

Table 8 shows that the failures in [27, p. 3] are located in two rows concerning singlets, respectively triplets, with conductors $f$ divisible by one prime which splits in the real quadratic field $K$ with 3 -class rank $\varrho=0$, respectively $\varrho=1$, that is, $m=1$, respectively $m=3$, and $t=s=1, n=0$. For $\varrho=0$, there are 10 fields of type $\delta_{2}$ too much, 9 fields of type $\beta_{2}$ too less, and 1 field of type $\varepsilon$ too less. They concern [27, Part I, $\varrho=0$, Section 3.1-5 and 3.14] and are corrected in Table 9. For $\varrho=1$, there is 1 field of type $\delta_{1}$ too much, which is of correct type $\beta_{1}$. Consequently, there is a corresponding erroneous impact on the row "total". Wrong counters in [27] are printed with boldface font.

Table 9: Corrections in the range $0<d_{L}<2 \cdot 10^{5}$.

\begin{tabular}{|r|r|r||c|c|}
\hline No. & $d_{L}$ & $f$ & $\begin{array}{r}\text { Erroneous Type } \\
{[27]}\end{array}$ & $\begin{array}{c}\text { Correct Type } \\
\tau(L)\end{array}$ \\
\hline 1 & 96481 & 7 & $\delta_{2}$ & $\beta_{2}$ \\
2 & 98833 & 7 & $\beta_{2}$ & $\varepsilon$ \\
3 & 160377 & 7 & $\varepsilon$ & $\beta_{2}$ \\
4 & 179144 & 7 & $\delta_{2}$ & $\beta_{2}$ \\
\hline 5 & 130329 & 9 & $\beta_{2}$ & $\varepsilon$ \\
\hline 6 & 65741 & 13 & $\delta_{2}$ & $\beta_{2}$ \\
7 & 110357 & 13 & $\delta_{2}$ & $\beta_{2}$ \\
8 & 114413 & 13 & $\delta_{2}$ & $\beta_{2}$ \\
9 & 125736 & 13 & $\delta_{2}$ & $\beta_{2}$ \\
\hline 10 & 193857 & 19 & $\delta_{2}$ & $\beta_{2}$ \\
\hline 11 & 93217 & 31 & $\delta_{2}$ & $\beta_{2}$ \\
12 & 134540 & 31 & $\delta_{2}$ & $\beta_{2}$ \\
\hline 13 & 114005 & 151 & $\delta_{2}$ & $\beta_{2}$ \\
\hline
\end{tabular}

We also give arithmetical invariants of the single erroneous triplet explicitly: For $d=568 \equiv 1(\bmod 3), f=\ell=13 \equiv$ $1(\bmod 3)$ and $d_{L}=f^{2} \cdot d=95992$, we have the pure type $\left(\delta_{1}, \delta_{1}, \delta_{1}\right)$ in [27, Part III, $\varrho=1$, Section 2.2$]$ instead of the correct type $\left(\beta_{1}, \delta_{1}, \delta_{1}\right)$, since the Voronoi algorithm did not find an absolute principal factor and we concluded $A=0$ instead of the correct $A=1$. In the coarse classification of Schmithals [38], there is no difference between these two types, since both are characterized by $(+,+,+)$.

\subsection{Gain of arithmetical structure for $100000<d_{L}<200000$}

Over real quadratic fields $K$ with 3 -class rank $\varrho=0$, this range brings some most remarkable gains:

1. first doublet for $f=3 q, d \equiv 3(9)$, and first type $(\varepsilon, \varepsilon)\left(d=5277, q=2, d_{L}=189972\right)$,

2. first type $\gamma$ for $f=q \ell\left(d=21, q=2, \ell=43, f=86, d_{L}=155316\right.$, singlet $\left.(\gamma)\right)$,

3. first nilet for $f=9 \ell, d \equiv 2(3)(d=29, \ell=7, f=63, D=115101)$, 
4. first occurrence of a conductor divisible by two splitting primes, $s=2$, namely $f=9 \ell, d \equiv 1$ (3), exploited immediately by the first occurrence of type $\alpha_{3}$ which requires $s \geq 2$, and consequently the first verification of the Scholz conjecture with unexpected two-dimensional relative principal factorization instead of two-dimensional capitulation ( $d=37, \ell=7, f=63, d_{L}=146853$, singlet $\left(\alpha_{3}\right)$, see Theorems 6.2 and 9.2),

5. first occurrence of $f=q_{1} q_{2} q_{3}$ as a doublet of type $(\gamma, \gamma)\left(d=13, f=110, d_{L}=157300\right)$,

6. first occurrence of $f=3 q_{1} q_{2}, d \equiv 6(9)$, as a doublet of type $(\gamma, \gamma)$

$\left(d=213, q_{1}=2, q_{2}=5, f=30, d_{L}=191700\right)$,

7. first occurrence of $f=9 q_{1} q_{2}, d \equiv 1(3)$, as a singlet of type $(\gamma)$

$\left(d=13, q_{1}=2, q_{2}=5, f=90, d_{L}=105300\right)$,

8. first occurrence of $f=q_{1} q_{2} \ell$ as a doublet of type $(\gamma, \gamma)\left(d=37, f=70, d_{L}=181300\right)$,

9. first occurrence of $f=3 q \ell, d \equiv 3(9)$, as a doublet of type $(\gamma, \gamma)$

$\left(d=93, q=2, \ell=7, f=42, d_{L}=164052\right)$.

The following phenomena arise within 3-ring class fields $K_{f}, f>1$, over real quadratic fields $K$ with 3-class rank $\varrho=1$ :

1. first occurrence of $f=3, d \equiv 3(9)$, as triplets containing types $\beta_{1}, \delta_{1}, \varepsilon$

$\left(d=12081, d_{L}=108729\right.$, type $\left(\delta_{1}, \delta_{1}, \delta_{1}\right)$, and $d=19749, d_{L}=177741$, type $\left.\left(\beta_{1}, \beta_{1}, \varepsilon\right)\right)$,

2. first occurrence of $f=9, d \equiv 2(3)$, as a triplet $\left(\delta_{1}, \delta_{1}, \delta_{1}\right)\left(d=1901, d_{L}=153981\right)$,

3. first type $\varepsilon$ in a triplet with $f=\ell\left(d=3873, \ell=7, d_{L}=189777\right.$, type $\left.\left(\delta_{1}, \delta_{1}, \varepsilon\right)\right)$.

Example 5.1. Heterogeneous multiplets arise from $d=37$ with $\varrho=0$ and 3-Selmer space $V=\langle\eta\rangle$, where $\eta$ denotes the fundamental unit of $K=\mathbb{Q}(\sqrt{37})$. For (4), we have a singlet $\operatorname{Inv}\left(K_{63}\right)=\left[\emptyset ; \emptyset, \emptyset ;\left(\alpha_{3}\right)\right]$, corresponding to the divisors $(1 ; 7,9 ; 63)$ of $f=63$, since $V(7)=V(9)=0$. For $(8)$, we have a quartet $\operatorname{Inv}\left(K_{70}\right)=\left[\emptyset ;(\varepsilon), \emptyset, \emptyset ; \emptyset,\left(\beta_{2}\right), \emptyset ;(\gamma, \gamma)\right]$, corresponding to $(1 ; 2,5,7 ; 10,35,14 ; 70)$, the divisors of $f=70$, since $V(2)=V$ but $V(5)=V(7)=0$. Recall that $148=2^{2} \cdot 37$ is the well-known minimum of all non-cyclic positive cubic discriminants. Its type is the singlet $(\varepsilon)$. The type of $45325=35^{2} \cdot 37$ is the singlet $\left(\beta_{2}\right)$. Padding nilets $\emptyset$ illuminate the arithmetical structure.

\section{Classifying Ennola and Turunen's range $0<d_{L}<500000$}

The increasing contributions by new types of conductors $f$ in the range $0<d_{L}<5 \cdot 10^{5}$ enforce a splitting into Table 10 for $\varrho=0$ and Table 11 for $\varrho \in\{1,2\}$.

According to Table 11, the total number of all non-cyclic totally real cubic fields $L$ with discriminants $d<5 \cdot 10^{5}$ is 26330 . Together with 110 cyclic cubic fields in Table 12 the number is $\mathbf{2 6}$ 440,

According to Table 12, the number of cyclic cubic fields $L$ with discriminant $0<d_{L}<5 \cdot 10^{5}$ is 110, with 60 arising from singlets having conductors $f$ with a single prime divisor, and 50 from doublets having two prime divisors of the conductor f. (M denotes the multiplicity.)

Example 6.1. In Table 10, the second line with conductor $f=q$, a prime number $q \equiv 2(\bmod 3)$, lists 10515 nilets, starting with the formal cubic discriminant $f^{2} \cdot d=2^{2} \cdot 5=20$, which does not belong to an actual cubic field, and 4296 singlets with minimal discriminant $d_{L}=f^{2} \cdot d=2^{2} \cdot 37=148$ of an actual cubic field L. Theoretical justifications for these facts are given in [35, Theorem 4.1]: the 3-Selmer space $V_{3}=\langle\eta\rangle$ of the real quadratic field $K=\mathbb{Q}(\sqrt{d})$ is generated by the fundamental unit $\eta \in U_{K}$. In the case of a nilet, the 3 -ring space $\bmod q, V_{3}(q)$, is the null space of codimension $\delta_{3}(q)=1$ in $V_{3}$, since $\eta \notin \mathcal{O}_{q}$. In the case of a singlet, we have $V_{3}(q)=V_{3}$ with defect $\delta_{3}(q)=0$.

Since minor counting errors have occurred in the tables by Moser, Angell and Llorente/Quer (whereas the table by Ennola/Turunen was correct), we explicitly state the ultimate counters of totally real cubic fields $L$ in five ranges of discriminants $0<d_{L}<B$ with various upper bounds $B$.

Theorem 6.1. The number of cyclic, respectively non-Galois, respectively all, non-isomorphic totally real cubic fields $L$ with discriminants in the range $0<d_{L}<B$ is given by

1. 6 , respectively 38 , respectively 44 , for $B=1500$,

2. 51 , respectively 4753 , respectively 4804 , for $B=100000$, 
Table 10: Totally real cubic discriminants $d_{L}=f^{2} \cdot d$ in the range $0<d_{L}<5 \cdot 10^{5}$.

\begin{tabular}{|c|c|c|c|c|c|c|c|c|c|c|c|c|c|c|c|}
\hline \multirow[b]{2}{*}{$f$} & \multirow[b]{2}{*}{ Condition } & \multicolumn{5}{|c|}{ Multiplicity } & \multicolumn{8}{|c|}{ Differential Principal Factorization } & \multirow[b]{2}{*}{ Total } \\
\hline & & 0 & 1 & 2 & 3 & 4 & $\alpha_{1}$ & $\alpha_{3}$ & $\beta_{1}$ & $\beta_{2}$ & $\gamma$ & $\delta_{1}$ & $\delta_{2}$ & $\varepsilon$ & \\
\hline 1 & $\varrho_{3}=0$ & 133534 & & & & & & & & & & & & & 0 \\
\hline$q$ & $\equiv 2(3)$ & 10515 & 4296 & & & & & & & & & & & 4296 & 4296 \\
\hline 3 & $d \equiv 3(9)$ & 1339 & 573 & & & & & & & & & & & 573 & 573 \\
\hline 3 & $d \equiv 6(9)$ & 1364 & 554 & & & & & & & & & & & 554 & 554 \\
\hline 9 & $d \equiv 6(9)$ & 46 & 166 & & 7 & & & & & & & & & 187 & 187 \\
\hline 9 & $d \equiv 2(3)$ & 453 & 197 & & & & & & & & & & & 197 & 197 \\
\hline 9 & $d \equiv 1(3)$ & 468 & 178 & & & & & & & 45 & & & 116 & 17 & 178 \\
\hline$\ell$ & $\equiv 1(3)$ & 1530 & 539 & & & & & & & 124 & & & 374 & 41 & 539 \\
\hline$q_{1} q_{2}$ & & 147 & 159 & 10 & & & & & & & 161 & & & 18 & 179 \\
\hline $3 q$ & $d \equiv 3(9)$ & 102 & 107 & 8 & & & & & & & 107 & & & 16 & 123 \\
\hline $3 q$ & $d \equiv 6(9)$ & 89 & 109 & 14 & & & & & & & 109 & & & 28 & 137 \\
\hline $9 q$ & $d \equiv 6(9)$ & 2 & & 20 & 3 & & & & & & 39 & & & 10 & 49 \\
\hline $9 q$ & $d \equiv 2(3)$ & 31 & 41 & 1 & & & & & & & 41 & & & 2 & 43 \\
\hline $9 q$ & $d \equiv 1(3)$ & 30 & 39 & 3 & & & & & & 39 & & & & 6 & 45 \\
\hline$q \ell$ & & 83 & 129 & 6 & & & & & & 125 & 4 & & & 12 & 141 \\
\hline $3 \ell$ & $d \equiv 3(9)$ & 12 & 20 & 1 & & & & & & 20 & & & & 2 & 22 \\
\hline $3 \ell$ & $d \equiv 6(9)$ & 11 & 16 & 1 & & & & & & 16 & & & & 2 & 18 \\
\hline $9 \ell$ & $d \equiv 6(9)$ & & & 1 & & & & & & 2 & & & & & 2 \\
\hline $9 \ell$ & $d \equiv 2(3)$ & 4 & 8 & & & & & & & 8 & & & & & 8 \\
\hline $9 \ell$ & $d \equiv 1(3)$ & 3 & 1 & & & & & 1 & & & & & & & 1 \\
\hline$\ell_{1} \ell_{2}$ & & 1 & 2 & & & & & 2 & & & & & & & 2 \\
\hline$q_{1} q_{2} q_{3}$ & & & & 1 & & & & & & & 2 & & & & 2 \\
\hline $3 q_{1} q_{2}$ & $d \equiv 3(9)$ & & 2 & 3 & & & & & & & 8 & & & & 8 \\
\hline $3 q_{1} q_{2}$ & $d \equiv 6(9)$ & & 1 & 1 & & & & & & & 3 & & & & 3 \\
\hline $9 q_{1} q_{2}$ & $d \equiv 2(3)$ & & 2 & & & & & & & & 2 & & & & 2 \\
\hline $9 q_{1} q_{2}$ & $d \equiv 1(3)$ & & 1 & 1 & & & & & & & 3 & & & & 3 \\
\hline$q_{1} q_{2} \ell$ & & & 1 & 2 & & & & & & 1 & 4 & & & & 5 \\
\hline $3 q \ell$ & $d \equiv 3(9)$ & & 2 & 1 & & & & & & & 4 & & & & 4 \\
\hline $3 q \ell$ & $d \equiv 6(9)$ & & & 2 & & & & & & & 4 & & & & 4 \\
\hline $9 q \ell$ & $d \equiv 2(3)$ & & & 1 & & & & & & & 2 & & & & 2 \\
\hline & Subtotal & & 7143 & 77 & 10 & & & 3 & & 380 & 493 & & 490 & 5961 & 7327 \\
\hline
\end{tabular}

3. 70 , respectively 9945 , respectively 10015 , for $B=200000$,

4. 110 , respectively 26330 , respectively 26440 , for $B=500000$,

5. 501, respectively 592421 , respectively 592922 , for $B=10000000$.

Proof. See the tables in Sections $4-7$.

Recall that no examples of the types $\alpha_{2}$ and $\alpha_{3}$ occurred in Angell's range $0<d_{L}<10^{5}$, and type $\alpha_{2}$ remained unknown even in Ennola and Turunen's range $0<d_{L}<5 \cdot 10^{5}$. Since this problem is intimately connected with the Scholz Conjecture in Section 9, we now emphasize the following theorem.

Theorem 6.2. The minimal discriminants $d_{L}=f^{2} \cdot d$ of totally real cubic fields $L$ with conductor $f$ and quadratic fundamental discriminant $d$ such that $\tau(L)$ is one of the extremely rare differential principal factorization types $\alpha_{3}$, respectively $\alpha_{2}$, are given by

1. 146853 with $f=63=9 \cdot 7, s=2$, and $d=37, \varrho=0$ (unique field in a singlet, $m=1$ ), respectively

2. 966397 with $f=19, s=1$, and $d=2677, \varrho=1$ ( wo of the fields in a triplet, $m=3$ ).

Proof. The unique field $L$ with discriminant 146853 has been discovered in August 1991 already [27, Part I, $\varrho=0$, Section 6.1] and was confirmed in the row with conductor $f=9 \ell, d \equiv 1(\bmod 3)$, of Table 6 . According to Theorem 9.2, this field forms a singlet with DPF type $\alpha_{3}$. 
Table 11: Table 10 with $0<d_{L}<5 \cdot 10^{5}$ continued for $\varrho_{3} \geq 1$.

\begin{tabular}{|c|c|c|c|c|c|c|c|c|c|c|c|c|c|c|c|}
\hline \multirow[b]{2}{*}{$f$} & \multirow[b]{2}{*}{ Condition } & \multicolumn{5}{|c|}{ Multiplicity } & \multicolumn{8}{|c|}{ Differential Principal Factorization } & \multirow[b]{2}{*}{ Total } \\
\hline & & 0 & 1 & 2 & 3 & 4 & $\alpha_{1}$ & $\alpha_{3}$ & $\beta_{1}$ & $\beta_{2}$ & $\gamma$ & $\delta_{1}$ & $\delta_{2}$ & $\varepsilon$ & \\
\hline 1 & $\varrho_{3}=1$ & & 18378 & & & & & & & & & 18378 & & & 18378 \\
\hline$q$ & $\equiv 2(3)$ & 1603 & & & 92 & & & & 48 & & & 213 & & 15 & 276 \\
\hline 3 & $d \equiv 3(9)$ & 188 & & & 8 & & & & 7 & & & 15 & & 2 & 24 \\
\hline 3 & $d \equiv 6(9)$ & 190 & & & 6 & & & & 2 & & & 15 & & 1 & 18 \\
\hline 9 & $d \equiv 6(9)$ & 17 & & & 2 & & & & 2 & & & 3 & & 1 & 6 \\
\hline 9 & $d \equiv 2(3)$ & 54 & & & 1 & & & & & & & 3 & & & 3 \\
\hline 9 & $d \equiv 1(3)$ & 53 & & & 1 & & & & 1 & & & 2 & & & 3 \\
\hline$\ell$ & $\equiv 1(3)$ & 150 & & & 8 & & & & 5 & & & 16 & & 3 & 24 \\
\hline$q_{1} q_{2}$ & & 15 & & & 3 & & & & 9 & & & & & & 9 \\
\hline $3 q$ & $d \equiv 3(9)$ & 11 & & & 2 & & & & 3 & & & & & 3 & 6 \\
\hline $3 q$ & $d \equiv 6(9)$ & 18 & & & 2 & & & & 6 & & & & & & 6 \\
\hline $9 q$ & $d \equiv 6(9)$ & 1 & & & & & & & & & & & & & 0 \\
\hline $9 q$ & $d \equiv 2(3)$ & 1 & & & 1 & & & & 3 & & & & & & 3 \\
\hline $9 q$ & $d \equiv 1(3)$ & 3 & & & & & & & & & & & & & 0 \\
\hline$q \ell$ & & 10 & & & 1 & & & & 3 & & & & & & 3 \\
\hline $3 \ell$ & $d \equiv 3(9)$ & 1 & & & & & & & & & & & & & 0 \\
\hline $3 \ell$ & $d \equiv 6(9)$ & 1 & & & & & & & & & & & & & 0 \\
\hline 1 & $\varrho_{3}=2$ & & & & & 61 & 175 & & & & & 69 & & & 244 \\
\hline & Subtotal & & 18378 & & 127 & "61 & 175 & & 89 & & & 18714 & & 25 & 19003 \\
\hline & Total & & 25521 & 77 & 137 & $\bar{~} 61$ & 175 & 3 & 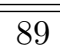 & 380 & 493 & 18714 & 490 & 5986 & 26330 \\
\hline
\end{tabular}

Table 12: Cyclic cubic discriminants $d_{L}=f^{2}$ in the range $0<d_{L}<5 \cdot 10^{5}$.

\begin{tabular}{|rl||rr||r|}
\hline \multicolumn{1}{|c||}{} & \multicolumn{2}{c||}{ M } & DPF \\
\hline 9 & $d=1$ & 1 & & $\zeta$ \\
$\ell$ & $\equiv 1(\bmod 3)$ & 59 & & 59 \\
\hline $9 \ell$ & $d=1$ & & 9 & 18 \\
$\ell_{1} \ell_{2}$ & $\equiv 1(\bmod 3)$ & & 16 & 32 \\
\hline & Summary & 60 & 25 & 110 \\
\hline
\end{tabular}

The triplet $\left(L_{1}, L_{2}, L_{3}\right)$ with discriminant 966397 was found by direct search on 19 November 2017. It is now confirmed by gapless construction in the row with conductor $f=\ell \equiv 1(\bmod 3)$ for $\varrho_{3}=1$ in Table 14 . According to Theorem 9.3, the DPF type of the triplet is $\left(\alpha_{2}, \alpha_{2}, \delta_{1}\right)$.

\subsection{Gain of arithmetical structure for $200000<d_{L}<500000$}

The following new features arise within 3-ring class fields $K_{f}, f>1$, over real quadratic fields $K$ with 3-class rank $\varrho=0$,

1. first doublet of type $(\varepsilon, \varepsilon)$ for $f=9 q, d \equiv 2(3)\left(d=1157, q=2, d_{L}=374868\right)$,

2. first doublet of type $(\varepsilon, \varepsilon)$ for $f=9 q, d \equiv 1(3)\left(d=877, q=2, d_{L}=284148\right)$,

3. first doublet of type $(\varepsilon, \varepsilon)$ for $f=3 \ell, d \equiv 3(9)\left(d=597, \ell=7, d_{L}=263277\right)$,

4. first doublet of type $(\varepsilon, \varepsilon)$ for $f=3 \ell, d \equiv 6(9)\left(d=1068, \ell=7, d_{L}=470988\right)$,

5. first occurrence of $f=9 \ell, d \equiv 6(9)$, as a doublet $\left(\beta_{2}, \beta_{2}\right)\left(d=60, \ell=7, d_{L}=238140\right)$,

6. first occurrence of $f=\ell_{1} \ell_{2}$ with $s=2$ as singlets of type $\left(\alpha_{3}\right)$

$\left(d=29, f=91, d_{L}=240149\right.$ and $\left.d=8, f=217, d_{L}=376712\right)$,

7. first singlet of type $(\gamma)$ for $f=3 q_{1} q_{2}, d \equiv 6(9)\left(d=357, f=30, d_{L}=321300\right)$, 
8. first occurrence of $f=9 q_{1} q_{2}, d \equiv 2(3)$, as singlet of type $(\gamma)\left(d=53, f=90, d_{L}=429300\right)$,

9. first singlet of type $\left(\beta_{2}\right)$ for $f=q_{1} q_{2} \ell\left(d=93, f=70, d_{L}=455700\right)$,

10. first singlet of type $(\gamma)$ for $f=3 q \ell, d \equiv 3(9)\left(d=165, f=42, d_{L}=291060\right)$,

11. first occurrence of $f=3 q \ell, d \equiv 6(9)$, as doublet of type $(\gamma, \gamma)\left(f=42, d_{L}=248724\right)$,

12. first occurrence of $f=9 q \ell, d \equiv 2(3)$, as doublet of type $(\gamma, \gamma)\left(d=29, d_{L}=460404\right)$.

The following phenomena arise within 3-ring class fields $K_{f}, f>1$, over $K$ with $\varrho=1$ :

1. first triplet of type $\left(\beta_{1}, \beta_{1}, \varepsilon\right)$ for $f=3, d \equiv 6(9)\left(d=52197, d_{L}=469773\right)$,

2. first occurrence of $f=9, d \equiv 6(9)$, as triplet $\left(\beta_{1}, \beta_{1}, \varepsilon\right)\left(d=5073, d_{L}=410913\right)$,

3. first occurrence of $f=9, d \equiv 1(3)$, as triplet $\left(\beta_{1}, \delta_{1}, \delta_{1}\right)\left(d=2917, d_{L}=236277\right)$,

4. first occurrence of $f=q_{1} q_{2}$, as triplet $\left(\beta_{1}, \beta_{1}, \beta_{1}\right)\left(d=3173, f=10, d_{L}=317300\right)$,

5. first occurrence of $f=3 q, d \equiv 3(9)$, as triplet $\left(\beta_{1}, \beta_{1}, \beta_{1}\right)\left(d=5637, f=6, d_{L}=202932\right)$,

6. first occurrence of $f=9 q, d \equiv 2(3)$, as triplet $\left(\beta_{1}, \beta_{1}, \beta_{1}\right)\left(d=1373, f=18, d_{L}=444852\right)$,

7. first occurrence of $f=q \ell$, as triplet $\left(\beta_{1}, \beta_{1}, \beta_{1}\right)\left(d=1101, f=14, d_{L}=215796\right)$.

First unramified quartet of type $\left(\delta_{1}, \delta_{1}, \delta_{1}, \delta_{1}\right)$ for $\varrho=2\left(d=d_{L}=214712[29,32]\right)$.

\section{Classifying Llorente and Quer's range $0<d_{L}<10000000$}

As opposed to the smaller ranges, the extension to Llorente and Quer's upper bound $10^{7}$ caused unexpected complications of two kinds. Firstly, for ramified extensions with conductor $f=2 \cdot 9=18, d \equiv 1(\bmod 3)$, at several discriminants $d_{L}=f^{2} \cdot d>4941972, d>15253, \varrho_{3}=0$, respectively $d_{L}=f^{2} \cdot d>4249908, d>13117, \varrho_{3}=1$. Secondly, for unramified extensions with $f=1$ and $\varrho_{3}=2$, at several discriminants $d_{L}=d>5547841$. Thus, we were very relieved, when a suitable work-around admitted the completion of the following most extensive and expensive Tables 13 and 14 on Wednesday, 13 January 2021.

According to Table 14, the total number of all non-cyclic totally real cubic fields $L$ with discriminants $d<10^{7}$ is 592421 . Together with 501 cyclic cubic fields in Table 15 the number is 592 922, in perfect accordance with Belabas [5, p. 1231 and Table 6.2, p. 1232], one field less than in the table of Llorente and Quer [24] (the unknown needle in a gigantic hay stack). We emphasize the difference between the number of discriminants (without multiplicities),

$$
559784+2231+5543+2879+5=\mathbf{5 7 0 4 4 2},
$$

and the number of pairwise non-isomorphic fields (including multiplicities in a weighted sum),

$$
1 \cdot 559784+2 \cdot 2231+3 \cdot 5543+4 \cdot 2879+6 \cdot 5=559784+4462+16629+11516+30=\mathbf{5 9 2} \mathbf{4 2 1},
$$

which is confirmed by adding the contributions to the 9 DPF types, $\alpha_{1}, \alpha_{2}, \alpha_{3}, \beta_{1}, \beta_{2}, \gamma, \delta_{1}, \delta_{2}, \varepsilon$,

$$
7951+142+122+3924+7639+9420+426972+11128+125123=\mathbf{5 9 2} \mathbf{4 2 1} .
$$

According to Table 15, the number of cyclic cubic fields $L$ with discriminant $0<d_{L}<10^{7}$ is 501, with 217 arising from singlets having conductors $f$ with a single prime divisor, 252 from doublets having two prime divisors of the conductor $f$, and 32 from quartets having three prime divisors of the conductor $f$. (M denotes the multiplicity.)

We point out that cyclic cubic fields are rather contained in ray class fields over $\mathbb{Q}$ than in ring class fields over real quadratic base fields. The single possible DPF type $\zeta$ has nothing to do with the 9 DPF types $\alpha_{1}, \alpha_{2}, \alpha_{3}, \beta_{1}, \beta_{2}, \gamma, \delta_{1}, \delta_{2}, \varepsilon$ of non-Abelian totally real cubic fields in [34]. 
Table 13: Totally real cubic discriminants $d_{L}=f^{2} \cdot d$ in the range $0<d_{L}<10^{7}$.

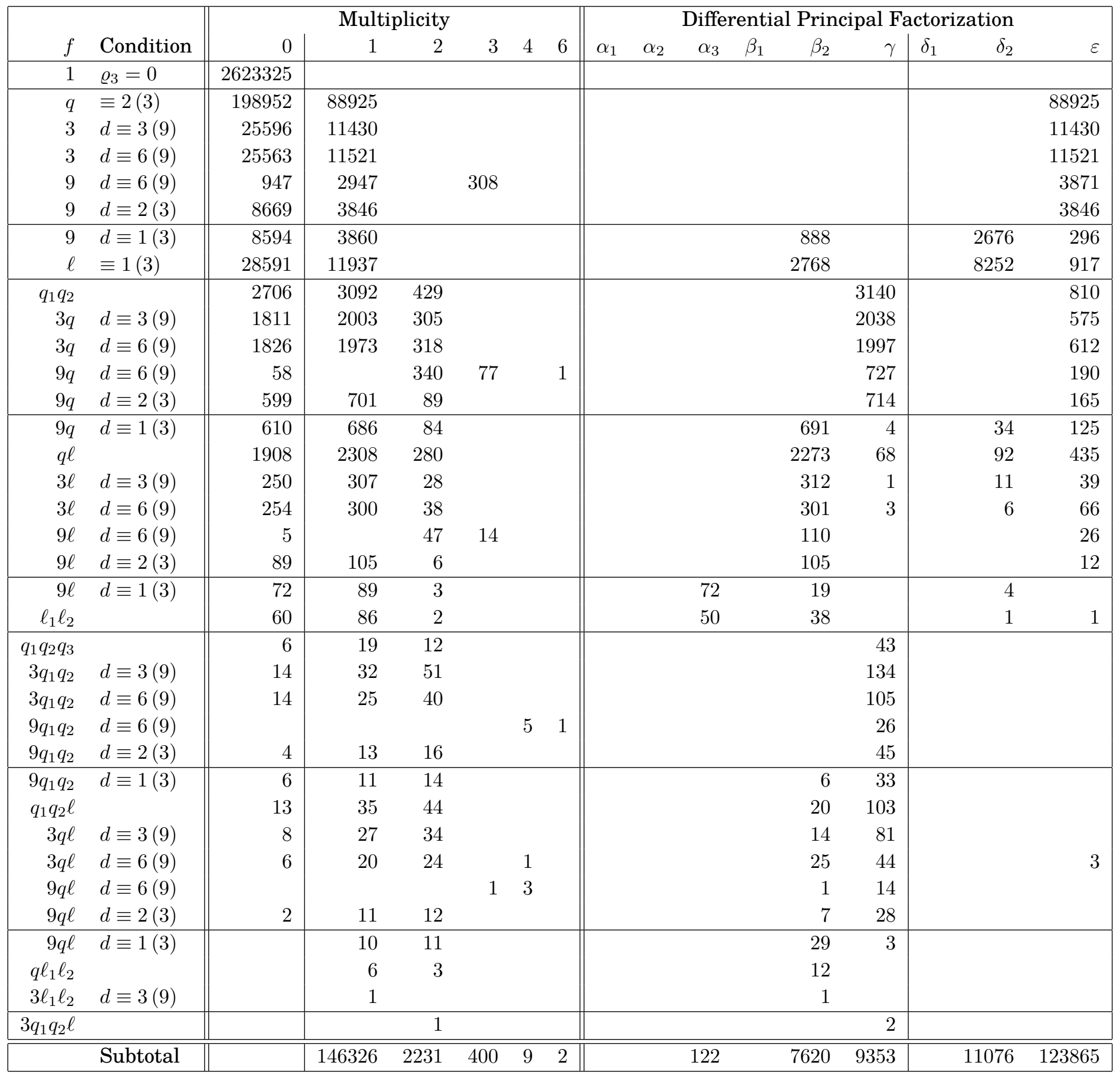

\subsection{Unramified Quartets}

According to Theorem 8.2, the 413458 unramified singlets $N / K$ with conductor $f=1$ over quadratic base fields $K$ with 3class rank $\varrho=\varrho_{3}(K)=1$ form an overwhelming crowd of colorless, monotonous, and boring fields which share the common type $\delta_{1}$.

In contrast, the 2870 unramified quartets $N / K$ over quadratic fields $K$ with $\varrho=2$ show an interesting statistical distribution of types. We consider the type $\left(\tau\left(L_{1}\right), \ldots, \tau\left(L_{4}\right)\right)$ of a quartet $\left(L_{1}, \ldots, L_{4}\right)$ as ordered lexicographically, regardless of permutations. Smallest $d$ see Table 16.

As known from [29] and [32], the 2391 quartets of mixed type $\left(\alpha_{1}, \alpha_{1}, \alpha_{1}, \delta_{1}\right)$ are extremely dominating with a relative frequency of $83.31 \%$. Moderate contributions are provided by the 234, respectively 175 , quartets of pure type $\left(\delta_{1}, \delta_{1}, \delta_{1}, \delta_{1}\right)$, respectively $\left(\alpha_{1}, \alpha_{1}, \alpha_{1}, \alpha_{1}\right)$. Quartets with mixed type $\left(\alpha_{1}, \delta_{1}, \delta_{1}, \delta_{1}\right)$ are rare with 62 hits, and the 8 quartets with mixed type $\left(\alpha_{1}, \alpha_{1}, \delta_{1}, \delta_{1}\right)$ are almost negligible. The reason for this behavior is well understood, because the corresponding capitulation types $\varkappa(K)=\left(\operatorname{ker}\left(T_{N_{1} / K}\right), \ldots, \operatorname{ker}\left(T_{N_{4} / K}\right)\right)$ enforce certain second 3-class groups $\mathrm{Gal}\left(\mathrm{F}_{3}^{2}(K) / K\right)$ of the quadratic base fields $K$ which can be realized easily for the quartets with high frequency, due to modest group orders, but require huge groups 
Table 14: Table 13 with $0<d_{L}<10^{7}$ continued for $\varrho_{3} \geq 1$.

\begin{tabular}{|c|c|c|c|c|c|c|c|c|c|c|c|c|c|c|c|c|}
\hline \multirow[b]{2}{*}{$f$} & \multirow[b]{2}{*}{ Condition } & \multicolumn{6}{|c|}{ Multiplicity } & \multicolumn{9}{|c|}{ Differential Principal Factorization } \\
\hline & & 0 & 1 & 2 & 3 & 4 & 6 & $\alpha_{1}$ & $\alpha_{2}$ & $\alpha_{3}$ & $\beta_{1}$ & $\beta_{2}$ & $\gamma$ & $\delta_{1}$ & $\delta_{2}$ & \\
\hline 1 & $\varrho_{3}=1$ & & 413458 & & & & & & & & & & & 413458 & & \\
\hline$q$ & $\equiv 2(3)$ & 38302 & & & 3239 & & & & & & 2022 & & & 6958 & & 737 \\
\hline 3 & $d \equiv 3(9)$ & 4798 & & & 375 & & & & & & 199 & & & 857 & & 69 \\
\hline 3 & $d \equiv 6(9)$ & 4760 & & & 359 & & & & & & 223 & & & 773 & & 81 \\
\hline 9 & $d \equiv 6(9)$ & 393 & & & 99 & & & & & & 61 & & & 211 & & 25 \\
\hline 9 & $d \equiv 2(3)$ & 1441 & & & 115 & & & & & & 83 & & & 241 & & 21 \\
\hline 9 & $d \equiv 1(3)$ & 1489 & & & 124 & & & & 27 & & 85 & & & 232 & 7 & 21 \\
\hline$\ell$ & $\equiv 1(3)$ & 4470 & & & 386 & & & & 95 & & 230 & 8 & & 706 & 33 & 86 \\
\hline$q_{1} q_{2}$ & & 534 & & & 115 & & & & & & 278 & & 12 & & & 55 \\
\hline $3 q$ & $d \equiv 3(9)$ & 370 & & & 78 & & 1 & & & & 187 & & 15 & 3 & & 35 \\
\hline $3 q$ & $d \equiv 6(9)$ & 399 & & & 84 & & 1 & & & & 174 & & 9 & 4 & & 71 \\
\hline $9 q$ & $d \equiv 6(9)$ & 13 & & & 25 & & 1 & & & & 69 & & & & & 12 \\
\hline $9 q$ & $d \equiv 2(3)$ & 111 & & & 20 & & & & & & 43 & & 5 & & & 12 \\
\hline $9 q$ & $d \equiv 1(3)$ & 117 & & & 24 & & & & 6 & & 57 & 3 & & & 3 & 3 \\
\hline$q \ell$ & & 364 & & & 67 & & & & 6 & & 160 & 6 & 2 & & 6 & 21 \\
\hline $3 \ell$ & $d \equiv 3(9)$ & 44 & & & 7 & & & & 3 & & 17 & 1 & & & & \\
\hline $3 \ell$ & $d \equiv 6(9)$ & 36 & & & 12 & & & & & & 24 & & & & 3 & 9 \\
\hline $9 \ell$ & $d \equiv 6(9)$ & & & & 4 & & & & & & 12 & & & & & \\
\hline $9 \ell$ & $d \equiv 2(3)$ & 12 & & & & & & & & & & & & & & \\
\hline $9 \ell$ & $d \equiv 1(3)$ & 12 & & & 1 & & & & 3 & & & & & & & \\
\hline$\ell_{1} \ell_{2}$ & & 4 & & & 1 & & & & 2 & & & 1 & & & & \\
\hline$q_{1} q_{2} q_{3}$ & & 1 & & & & & & & & & & & & & & \\
\hline $3 q_{1} q_{2}$ & $d \equiv 3(9)$ & 3 & & & 1 & & & & & & & & 3 & & & \\
\hline $3 q_{1} q_{2}$ & $d \equiv 6(9)$ & 4 & & & 4 & & & & & & & & 12 & & & \\
\hline $9 q_{1} q_{2}$ & $d \equiv 1(3)$ & & & & 1 & & & & & & & & 3 & & & \\
\hline$q_{1} q_{2} \ell$ & & & & & 2 & & & & & & & & 6 & & & \\
\hline $3 q \ell$ & $d \equiv 3(9)$ & 3 & & & & & & & & & & & & & & \\
\hline $3 q \ell$ & $d \equiv 6(9)$ & 4 & & & & & & & & & & & & & & \\
\hline 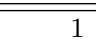 & 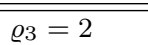 & & & & & 2870 & & 7951 & & & & & & 3529 & & \\
\hline$q$ & $\equiv 2(3)$ & 197 & & & & & & & & & & & & & & \\
\hline 3 & $d \equiv 3(9)$ & 19 & & & & & & & & & & & & & & \\
\hline 3 & $d \equiv 6(9)$ & 18 & & & & & & & & & & & & & & \\
\hline 9 & $d \equiv 2(3)$ & 3 & & & & & & & & & & & & & & \\
\hline 9 & $d \equiv 1(3)$ & 3 & & & & & & & & & & & & & & \\
\hline$\ell$ & $\equiv 1(3)$ & 6 & & & & & & & & & & & & & & \\
\hline $3 q$ & $d \equiv 3(9)$ & 3 & & & & & & & & & & & & & & \\
\hline $3 q$ & $d \equiv 6(9)$ & 1 & & & & & & & & & & & & & & \\
\hline & Subtotal & & 413458 & 0 & 5143 & 2870 & 3 & 7951 & 142 & 0 & 3924 & 19 & 67 & 426972 & 52 & 1258 \\
\hline & Total & & $\overline{559784}$ & 2231 & 5543 & 2879 & 5 & 7951 & 142 & 122 & 3924 & 7639 & 9420 & 426972 & 11128 & 125123 \\
\hline
\end{tabular}

in the case of rare quartets (see [30]).

\subsection{Other multiplets}

According to Table 14, the number 2231 of doublets, respectively 5543 of triplets, respectively 2879 of quartets, respectively 5 of sextets, agrees with the corresponding counters given in [24, Table 2, p. 588], respectively [24, Table 3, p. 589], respectively [24, Table 4, p. 589], respectively [24, p. 588 and Table 5, p. 590], in the paper by Llorente and Quer. However, there are two misprints in the text below Table 4 on page 589 of [24], where the authors intended to state that 2870 among the 2879 quartets belong to real quadratic fields $K$ with 3 -class rank $\varrho=2$, namely the unramified quartets in our Table 16. But the remaining 9 quartets are ramified over real quadratic fields $K$ with 3 -class rank $\varrho=0$ and show up in our Table 13. They are analyzed in detail in the following example.

Example 7.1. A common feature of all 9 ramified quartets $\left(L_{1}, \ldots, L_{4}\right)$ with discriminants in the range $0<d_{L}<10^{7}$ is the congruence class of the quadratic fundamental discriminant $d \equiv 6(\bmod 9)$ which enables both, conductors with 3contribution $v_{3}(f)=1$ and $v_{3}(f)=2$. The reason for their multiplicity in terms of 3-defects $\delta_{3}(f)$ (co-dimensions of 3-ring spaces $V_{3}(f)$ ) was discussed in [28, Supplements Section, Part 1.a, p. S55, and Part 2.d, pp. S57-S58]. Now we are able to present their differential principal factorizations in Table 17, where the type of the conductor establishes the connection with Table 13. A generating polynomial for each member L of the quartets is given in [24, Table 6, p. 591], but we point out that the conductor in the caption of this table should be $T=3^{m} T_{0}>1$ (our $f$ ), and the discriminant in the table header should be $D=3^{2 m} T_{0}^{2} d$ (our $d_{L}$ ). 
Example 7.2. A particular highlight of the range $0<d_{L}<10^{7}$ is the occurrence of 5 sextets, which did not show up in smaller tables. The reason for their multiplicity in terms of 3-defects $\delta_{3}(f)$ (co-dimensions of 3-ring spaces $V_{3}(f)$ modulo $f$ in the 3-Selmer space $V_{3}$ ) was discussed in [28, Supplements Section, Part 1.c, p. S56, Part 2.b, p. S57, Part 2.d, pp. S57-S58, and Part 2.f, $p$. S58]. Now we are able to present their differential principal factorizations in Table 18. The leading two sextets are mixed, and the trailing three sextets are pure. The constitution of the sextets is very heterogeneous: although four of the quadratic fundamental discriminants $d$ admit the irregular contribution 9 to the conductor $f$ only three conductors are actually divisible by 9, but they differ either by the 3-rank @ or by the kind of the conductor. A generating polynomial for each member $L$ of the sextets is given in [24, Table 5, p. 590], but again we point out that the discriminant in the table header should be $D=3^{2 m} T_{0}^{2} d$ (our $d_{L}=f^{2} \cdot d$ ). Types for $\varrho=0$ are more simple.

Example 7.3. We split the 2231 doublets in the range $0<d_{L}<10^{7}$ according to the shape of $f$.

- In Table 19, we begin with two non-split prime divisors of $f$, that is, we accumulate the results for $f=q_{1} q_{2}, f=3 q$ with $d \equiv \pm 3(\bmod 9)$, and $f=9 q$ with $d \equiv 2(\bmod 3)$. The DPF type $(\varepsilon, \varepsilon)$ is highly dominating over $(\gamma, \varepsilon)$ and $(\gamma, \gamma)$. Here and in the sequel, the given paradigms for $d_{L}$ are not necessarily minimal. Note the constitution $1141=429+305+318+89$ of the total frequency.

- The irregular situation $f=9 q$ with $d \equiv 6(\bmod 9)$ in Table 20 shows a reversal of tendencies. DPF type $(\gamma, \gamma)$ is dominating, $(\varepsilon, \varepsilon)$ remains moderate, mixed type $(\gamma, \varepsilon)$ is almost negligible.

- Table 21 reveals that, for $f=q \ell, f=3 \ell$ with $d \equiv \pm 3(\bmod 9), f=9 \ell$ with $d \equiv 2(\bmod 3)$, and $f=9 q$ with $d \equiv 1(\bmod 3)$, DPF type $(\varepsilon, \varepsilon)$ prevails, followed by $\left(\delta_{2}, \delta_{2}\right)$.

- Again, the irregular situation $f=9 \ell$ with $d \equiv 6(\bmod 9)$ in Table 22 shows a reversal of tendencies. DPF type $\left(\beta_{2}, \beta_{2}\right)$ dominates over $(\varepsilon, \varepsilon)$.

- In the case of three non-split prime divisors of $f$, i.e., $f=q_{1} q_{2} q_{3}$ or $f=3 q_{1} q_{2}$ with $d \equiv \pm 3(\bmod 9)$ or $f=9 q_{1} q_{2}$ with $d \equiv 2(\bmod 3)$, no table is required, since all $119=12+51+40+16$ occurrences are of type $(\gamma, \gamma)$, e.g. $d=93, f=30$, $d_{L}=83700$.

Example 7.4. We split the 5543 triplets in the range $0<d_{L}<10^{7}$ according to the shape of $f$.

- Triplets are usually due to elevated 3-class rank $\varrho \geq 1$ of the real quadratic field $K$. However, the simplest case of triplets with $\varrho=0$ arises for the irregular prime power conductor $f=9, d \equiv 6(\bmod 9)$. The minimal occurrence is $d=717$, $d_{L}=58077$. Each of the 308 triplets is embedded in a heterogeneous quartet $\operatorname{Inv}\left(K_{9}\right)=[(\varepsilon),(\varepsilon, \varepsilon, \varepsilon)]$.

- There are 77 cases of triplets with irregular conductors $f=9 q, d \equiv 6(\bmod 9)$, with $\varrho=0$. They are all of pure type $(\gamma, \gamma, \gamma)$, for instance $d=69, q=2, f=18, d_{L}=22356$.

- For the irregular case $f=9 \ell, d \equiv 6(\bmod 9)$, with $\varrho=0$, all 14 occurrences are of type $\left(\beta_{2}, \beta_{2}, \beta_{2}\right)$, for instance $d=177$, $\ell=7, d_{L}=702513$. There always exists an associated singlet of type $(\varepsilon)$ with conductor $f=3$, that is, the the triplet and the singlet are embedded in a heterogeneous quartet $\operatorname{Inv}\left(K_{9 \ell}\right)=\left[\emptyset,(\varepsilon), \emptyset, \emptyset, \emptyset,\left(\beta_{2}, \beta_{2}, \beta_{2}\right)\right]$ corresponding to the divisors $(1,3,9, \ell, 3 \ell, 9 \ell)$ of $f$.

- A unique example of $f=9 q \ell, d \equiv 6(\bmod 9)$, with $\varrho=0$, is given by $d=69, q=2, \ell=13, f=234, d_{L}=3778164$. It is a triplet of mixed type $\left(\beta_{2}, \gamma, \gamma\right)$.

- For $\varrho=1$ and non-critical split $f=\ell \equiv 1(\bmod 3)$, the mixed DPF type $\left(\beta_{1}, \delta_{1}, \delta_{1}\right)$ prevails, followed by mixed type $\left(\delta_{1}, \delta_{1}, \varepsilon\right)$. Mixed types have only two distinct components. See Table 23.

- For $\varrho=1$ and critical split $f=9, d \equiv 1(\bmod 3)$, again the mixed DPF type $\left(\beta_{1}, \delta_{1}, \delta_{1}\right)$ prevails, followed by mixed type $\left(\delta_{1}, \delta_{1}, \varepsilon\right)$. Here, all examples have minimal discriminant $d_{L}$. See Table 24 .

Table 15: Cyclic cubic discriminants $d_{L}=f^{2}$ in the range $0<d_{L}<10^{7}$.

\begin{tabular}{|rl||rrr||rrr|}
\hline \multicolumn{1}{|c||}{} & \multicolumn{3}{c|}{ M } & DPF & $f$ & $d_{L}$ \\
\hline 9 & Condition & 1 & 2 & 4 & $\zeta$ & & \\
$\ell$ & $\equiv 1(\bmod 3)$ & 1 & & & 1 & 9 & 81 \\
\hline $9 \ell$ & $d=1$ & & & 216 & 7 & 49 \\
$\ell_{1} \ell_{2}$ & $\equiv 1(\bmod 3)$ & & 33 & & 66 & 63 & 3969 \\
$9 \ell_{1} \ell_{2}$ & $d=1$ & & & 6 & 24 & 91 & 8281 \\
$\ell_{1} \ell_{2} \ell_{3}$ & $\equiv 1(\bmod 3)$ & & & 2 & 8 & 1729 & 2989441 \\
\hline & Summary & 217 & 126 & 8 & 501 & & \\
\hline
\end{tabular}


- For $\varrho=1$ and non-split $f=q \equiv 2(\bmod 3)$ or $f=3$ or $f=9, d \equiv 2(\bmod 3)$, the pure DPF type $\left(\delta_{1}, \delta_{1}, \delta_{1}\right)$ is dominating, followed by the mixed type $\left(\beta_{1}, \beta_{1}, \varepsilon\right)$. Mixed type $\left(\beta_{1}, \delta_{1}, \varepsilon\right)$ with three distinct components is very rare. See Table 25 , where $4088=3239+359+375+115$.

- Table 26 gives the distribution of DPF types for $f=q_{1} q_{2}, f=3 q$, and $f=9 q, d \equiv 2(\bmod 3)$, where $297=115+78+84+20$. Pure DPF type $\left(\beta_{1}, \beta_{1}, \beta_{1}\right)$ prevails, followed by pure type $(\varepsilon, \varepsilon, \varepsilon)$.

- Table 27 shows the triplets with $f=q \ell, f=3 \ell$, and $f=9 q, d \equiv 1(\bmod 3)$. (There are no hits for $f=9 \ell, d \equiv 2(\bmod 3)$.) The pure DPF type $\left(\beta_{1}, \beta_{1}, \beta_{1}\right)$ prevails, followed by pure type $(\varepsilon, \varepsilon, \varepsilon)$. Here, $110=67+7+12+24$.

- There are only two triplets with two split ramified primes:

mixed type $\left(\alpha_{2}, \alpha_{2}, \beta_{2}\right)$ for $f=\ell_{1} \ell_{2}\left(d=940, f=91, d_{L}=7784140\right)$, and

pure type $\left(\alpha_{2}, \alpha_{2}, \alpha_{2}\right)$ for $f=9 \ell, d \equiv 1(\bmod 3)\left(d=2101, f=63, d_{L}=8338869\right)$.

We conclude this section on multiplets with information on singlets.

\section{Theorem 7.1. (Ramified and unramified singlets)}

1. A ramified singlet (with conductor $f>1$ ) can only be of type $\left(\alpha_{3}\right),\left(\beta_{2}\right),(\gamma),\left(\delta_{2}\right),(\varepsilon)$.

2. An unramified singlet (with conductor $f=1$ ) must be of type $\left(\delta_{1}\right)$.

Proof. According to the fundamental inequalities in Corollary 3.2 and the fundamental equation in Corollary 3.1, we have:

1. For $f>1$, the multiplicity formula shows that $3^{\varrho}$ divides $m$ [31, Theorem 3.2, p. 2215, Theorem 3.3-3.4, p. 2217, and Theorem 4.1-4.2, p. 2224-2225]. Thus, a singlet can only occur for $\varrho=0$, and this implies $C=0$, i.e. no capitulation can happen. By Theorem 3.6, we conclude $\tau(L) \notin\left\{\alpha_{1}, \alpha_{2}, \beta_{1}, \delta_{1}\right\}$, and consequently $\tau(L) \in\left\{\alpha_{3}, \beta_{2}, \gamma, \delta_{2}, \varepsilon\right\}$.

2. For $f=1$, we have the multiplicity formula $m=\left(3^{\varrho}-1\right) / 2$ [31, Theorem 3.1, p. 2214]. Therefore, a singlet with $m=1$ occurs for $\varrho=1$. On the other hand, $f=1$ implies $t=s=0$, and thus $A=R=0$. The fundamental equation degenerates to $U+1=C$, where $\varrho=1$ implies the bound $C \leq 1$. Thus, $C=1$ and $U=0$, that is the unique type $\delta_{1}$.

Example 7.5. Indeed, singlets of all the types in Theorem 7.1 actually do occur. Their minimal discriminants $d_{L}$ are given in Table 28.

Concerning the frequency of singlets for $0<d_{L}<10^{7}$, the first row in Table 14 proves that unramified singlets $\left(\delta_{1}\right)$ form the definite hichamp 413458 among all contributions. The last row (Subtotal) in Table 13 illuminates the second extreme contribution 146326 by all the other ramified singlets $\left(\alpha_{3}\right),\left(\beta_{2}\right),(\gamma),\left(\delta_{2}\right)$, and clearly dominating $(\varepsilon)$.

Another interesting observation is enabled by the rows with regular conductors $f$ divisible by exactly two primes, i.e. $t=2$, in Table 13. It appears that, under certain conditions, non-split extensions $N / K$ with $U_{K}=N_{N / K}\left(U_{N}\right)$ in the sense of Remark 3.3 are forbidden. Generalizing a proof for singlets of type $(\gamma)$ by Moser at the top of p. 74 in [36], we partition the case $t=2$, according to the number $0 \leq s \leq 2$ of prime divisors of $f$ which split in the real quadratic field $K$. The crucial assumption $\varrho=0$, that is, the class number of $K$ is not divisible by 3 (and thus capitulation is discouraged, $C=0$ ), implies that there are only three possible types of split extensions $N / K$, namely the singlets $\left(\alpha_{3}\right),\left(\beta_{2}\right),(\gamma)$.

Theorem 7.2. (Ramified singlets of split extensions $N / K$ )

Suppose that $K$ is a real quadratic field with 3-class rank $\varrho=0$, and let $f$ be a regular 3-admissible conductor for $K$ with exactly two restrictive prime divisors, $t=2$. Denote by $0 \leq s \leq 2$ the number of prime divisors of $f$ which split in the real quadratic field $K$. Then the following conditions enforce a split extension $N / K$ with $U_{N}=U_{K} \cdot E_{N / K}$, where $E_{N / K}=U_{N} \cap \operatorname{ker}\left(N_{N / K}\right)$ denotes the subgroup of relative units of $N / K$.

Table 16: Types of unramified quartets in the range $0<d_{L}<10^{7}$.

\begin{tabular}{|c|c|c|c|}
\hline $\begin{array}{c}\text { DPF Type } \\
\left(\tau\left(L_{1}\right), \ldots, \tau\left(L_{4}\right)\right)\end{array}$ & $\begin{array}{l}\text { Capitulation Number } \nu(K) \\
\text { (according to [8]) }\end{array}$ & Frequency & $d_{L}=d$ \\
\hline$\left(\alpha_{1}, \alpha_{1}, \alpha_{1}, \alpha_{1}\right)$ & 4 & 175 & 62501 \\
\hline$\left(\alpha_{1}, \alpha_{1}, \alpha_{1}, \delta_{1}\right)$ & 3 & 2391 & 32009 \\
\hline$\left(\alpha_{1}, \alpha_{1}, \delta_{1}, \delta_{1}\right)$ & 2 & 8 & 710652 \\
\hline$\left(\alpha_{1}, \delta_{1}, \delta_{1}, \delta_{1}\right)$ & 1 & 62 & 534824 \\
\hline$\left(\delta_{1}, \delta_{1}, \delta_{1}, \delta_{1}\right)$ & 0 & 234 & 214712 \\
\hline & Total: & 2870 & \\
\hline
\end{tabular}


Table 17: Nine explicit ramified quartets in the range $0<d_{L}<10^{7}$.

\begin{tabular}{|r|r||r|r|c||c|}
\hline No. & $d_{L}$ & $d$ & $f$ & Kind of Conductor & $\begin{array}{c}\text { DPF Type } \\
\left(\tau\left(L_{1}\right), \ldots, \tau\left(L_{4}\right)\right)\end{array}$ \\
\hline 1 & 1725300 & 213 & $90=9 \cdot 2 \cdot 5$ & $9 q_{1} q_{2}$ & $(\gamma, \gamma, \gamma, \gamma)$ \\
2 & 2238516 & 141 & $126=9 \cdot 2 \cdot 7$ & $9 q \ell$ & $(\gamma, \gamma, \gamma, \gamma)$ \\
3 & 2891700 & 357 & $90=9 \cdot 2 \cdot 5$ & $9 q_{1} q_{2}$ & $(\gamma, \gamma, \gamma, \gamma)$ \\
4 & 4641300 & 573 & $90=9 \cdot 2 \cdot 5$ & $9 q_{1} q_{2}$ & $(\gamma, \gamma, \gamma, \gamma)$ \\
5 & 6810804 & 429 & $126=9 \cdot 2 \cdot 7$ & $9 q \ell$ & $(\gamma, \gamma, \gamma, \gamma)$ \\
6 & 7557300 & 933 & $90=9 \cdot 2 \cdot 5$ & $9 q_{1} q_{2}$ & $(\gamma, \gamma, \gamma, \gamma)$ \\
7 & 7953876 & 501 & $126=9 \cdot 2 \cdot 7$ & $9 q \ell$ & $(\gamma, \gamma, \gamma, \gamma)$ \\
8 & 8250228 & 4677 & $42=3 \cdot 2 \cdot 7$ & $3 q \ell$ & $(\gamma, \varepsilon, \varepsilon, \varepsilon)$ \\
9 & 8723700 & 1077 & $90=9 \cdot 2 \cdot 5$ & $9 q_{1} q_{2}$ & $(\gamma, \gamma, \gamma, \gamma)$ \\
\hline
\end{tabular}

1. If $s=0$ and $N$ has 3 -class number 1 , then $N$ is a singlet of type $(\gamma)$.

2. If $s=1$ and $N$ has 3-class number 3 , then $N$ is a singlet of type $\left(\beta_{2}\right)$.

3. If $s=2$ and $N$ has 3 -class number 9 , then $N$ is a singlet of type $\left(\alpha_{3}\right)[$ or $(\gamma)]$.

Proof. The assumption $\varrho=0$ implies that the 3-Selmer space $V$ of $K$ is generated by the fundamental unit $\eta$ of $K$. Since we suppose $t=2$ with regular restrictive prime divisors $q_{1}, q_{2}$ of the conductor $f$, in the sense of Remark 8.1, $\eta$ is not contained in the 3-ring spaces $V\left(q_{1}\right)$ and $V\left(q_{2}\right)$, and the multiplicity of $f$ is $m=1$, i.e., we have a singlet [31, Theorem 3.3, p. 2217].

According to [36, Theorem A, p. 70], the subgroup $\mathrm{Cl}_{N}^{\sigma}$ of weakly ambiguous ideal classes of $\mathrm{Cl}_{N}$, with respect to $\operatorname{Gal}(N / K)=\langle\sigma\rangle$, is of order $\# \mathrm{Cl}_{3}(K) \cdot 3^{T-1} / 3^{Q}$, where the norm index is denoted by $3^{Q}=\left(U_{K}:\left(U_{K} \cap N_{N / K}\left(N^{\times}\right)\right)\right)$and $T$ is the number of prime ideals of $K$ which ramify in $N$. In our situation, we have $\# \mathrm{Cl}_{3}(K)=1$, and $\# \mathrm{Cl}_{3}(N)=3^{s}$ is divisible by $3^{T-1-Q}$, where $T=2+s$, that is, $s \geq 1+s-Q$, respectively $Q \geq 1$ and thus $Q=1$. A fortiori, the unit norm index is $b=\left(U_{K}: N_{N / K}\left(U_{N}\right)\right)=\left(U_{K}:\left(U_{K} \cap N_{N / K}\left(N^{\times}\right)\right)\right) \cdot\left(\left(U_{K} \cap N_{N / K}\left(N^{\times}\right)\right): N_{N / K}\left(U_{N}\right)\right)=3$, because $\eta$ is not norm of a number in $N^{\times}$, let alone of a unit in $U_{N}$. Thus, $N / K$ is a split extension, in the sense of Remark 3.3, and the types $\left(\delta_{1}\right)$ and $(\varepsilon)$ in item (1) of Theorem 7.1 are impossible.

Equation (16) in additive form, $V_{N}=2 \cdot V_{L}+V_{K}+E-2$, where $V_{F}:=v_{3}\left(\# \mathrm{Cl}_{F}\right)$ for a number field $F$, degenerates to $V_{N}=2 \cdot V_{L}+E-2$ under our assumption $V_{K}=0$. This gives rise to a parity condition for $E$ :

1. If $s=0$ and $V_{N}=0$, then $2 \cdot V_{L}=2-E$ and $E$ must be even, $E=2, N$ of type $(\gamma)$.

2. If $s=1$ and $V_{N}=1$, then $2 \cdot V_{L}=3-E$ and $E$ must be odd, $E=1, N$ of type $\left(\beta_{2}\right)$.

3. If $s=2$ and $V_{N}=2$, then $2 \cdot V_{L}=4-E$ and $E$ must be even, [either] $E=0, N$ of type $\left(\alpha_{3}\right)$ [or $E=2, N$ of type $(\gamma)$, conjectured impossible].

\section{Statistical evaluation and theoretical interpretation of the tables}

Now we illuminate and analyze our extensive numerical (computational, experimental) results with the aid of statistical evaluations and theoretical statements.

Table 18: Five explicit sextets in the range $0<d_{L}<10^{7}$.

\begin{tabular}{|r|r||r|r|r|c||c|}
\hline No. & $d_{L}$ & $d$ & $\varrho$ & $f$ & Kind of Conductor & $\begin{array}{c}\text { DPF Type } \\
\left(\tau\left(L_{1}\right), \ldots, \tau\left(L_{6}\right)\right)\end{array}$ \\
\hline 1 & 3054132 & $84837 \equiv 3(9)$ & 1 & $6=3 \cdot 2$ & $3 q$ & $\left(\beta_{1}, \beta_{1}, \delta_{1}, \delta_{1}, \delta_{1}, \varepsilon\right)$ \\
2 & 4735467 & $131541 \equiv 6(9)$ & 1 & $6=3 \cdot 2$ & $3 q$ & $\left(\beta_{1}, \delta_{1}, \delta_{1}, \delta_{1}, \delta_{1}, \varepsilon\right)$ \\
3 & 5807700 & $717 \equiv 6(9)$ & 0 & $90=9 \cdot 2 \cdot 5$ & $9 q_{1} q_{2}$ & $(\gamma, \gamma, \gamma, \gamma, \gamma, \gamma)$ \\
4 & 6367572 & $19653 \equiv 6(9)$ & 1 & $18=9 \cdot 2$ & $9 q$ & $\left(\beta_{1}, \beta_{1}, \beta_{1}, \beta_{1}, \beta_{1}, \beta_{1}\right)$ \\
5 & 9796788 & $30237 \equiv 6(9)$ & 0 & $18=9 \cdot 2$ & $9 q$ & $(\varepsilon, \varepsilon, \varepsilon, \varepsilon, \varepsilon, \varepsilon)$ \\
\hline
\end{tabular}


Table 19: Types of doublets with two non-split prime divisors of regular $f$.

\begin{tabular}{|c||r||rrr|}
\hline DPF Type & Frequency & $d$ & $f$ & $d_{L}$ \\
$\left(\tau\left(L_{1}\right), \tau\left(L_{2}\right)\right)$ & & & & \\
\hline$(\gamma, \gamma)$ & 40 & 33 & 45 & 66825 \\
$(\gamma, \varepsilon)$ & 40 & 9973 & 10 & 997300 \\
$(\varepsilon, \varepsilon)$ & 1061 & 373 & 10 & 37300 \\
\hline Total: & 1141 & & & \\
\hline
\end{tabular}

Table 20: Types of doublets with two non-split prime divisors of irregular $f$.

\begin{tabular}{|c||r||rrr|}
\hline DPF Type & Frequency & $d$ & $f$ & $d_{L}$ \\
$\left(\tau\left(L_{1}\right), \tau\left(L_{2}\right)\right)$ & & & & \\
\hline$(\gamma, \gamma)$ & 245 & 213 & 18 & 69012 \\
$(\gamma, \varepsilon)$ & 6 & 9213 & 18 & 2985012 \\
$(\varepsilon, \varepsilon)$ & 89 & 141 & 18 & 45684 \\
\hline Total: & 340 & & & \\
\hline
\end{tabular}

\subsection{Stagnation and evolution of arithmetical structures}

Some features in the Tables 1, 6, 10, 11, 13, and 14 reveal stagnation, that is, multiplicities and DPF types remain constant, and only the statistical counters show monotonic growth, usually slightly faster than linear. Other phenomena stick out with conspicuous evolution, leading to new multiplicities and new DPF types. The huge total number 592922 of all objects occurring in our investigation of the extensive range $0<d_{L}<10^{7}$ admits sound statistical interpretation and heuristic predictions in unproven conjectures.

Since the conductor $f=1$ is 3 -admissible for any quadratic fundamental discriminant $d$, the quadratic fields $K=\mathbb{Q}(\sqrt{d})$ with 3-class rank $\varrho=0$ must be considered as giving rise to nilets $\mathbf{M}_{d}=\emptyset$. Observe that the 3-ring space $V(1)$ modulo 1 coincides with 3-Selmer space $V$ and the multiplicity formula for the unramified situation yields $m(d)=m\left(1^{2} \cdot d\right)=$ $\frac{1}{2}\left(3^{\varrho}-1\right)=\frac{1}{2}\left(3^{0}-1\right)=0$.

In the following conjectures, of which certain parts are proven theorems, we always give successive percentages with respect to the upper bounds $10^{5}, 2 \cdot 10^{5}, 5 \cdot 10^{5}$ and $10^{7}$, in this order.

Conjecture 8.1. The relative frequency of unramified nilets with $\varrho=0$ slightly decreases from $89.1 \%$ over $88.6 \%$ and $87.9 \%$ to $86.3 \%$. The relative frequency of unramified singlets with $\varrho=1$ slightly increases from $10.9 \%$ over $11.4 \%$ and $12.1 \%$ to 13.6\%. All singlets are of permanent type $\delta_{1}$, showing stagnation. See Theorems 8.2 and 8.6. The relative frequency of unramified quartets with $\varrho=2$ is marginal below $0.1 \%$, but they reveal an interesting evolution of types:

1. Up to $10^{5}, \frac{4}{5}=80 \%$ are of mixed type $\left(\alpha_{1}, \alpha_{1}, \alpha_{1}, \delta_{1}\right), \frac{1}{5}=20 \%$ of pure type $\left(\alpha_{1}, \alpha_{1}, \alpha_{1}, \alpha_{1}\right)$.

2. Up to $2 \cdot 10^{5}, \frac{14}{16}=87.5 \%$ are of type $\left(\alpha_{1}, \alpha_{1}, \alpha_{1}, \delta_{1}\right), \frac{2}{16}=12.5 \%$ of type $\left(\alpha_{1}, \alpha_{1}, \alpha_{1}, \alpha_{1}\right)$.

3. Up to $5 \cdot 10^{5}, \frac{53}{16}=86.9 \%$ are of mixed type $\left(\alpha_{1}, \alpha_{1}, \alpha_{1}, \delta_{1}\right), \frac{4}{61}=6.6 \%$ of pure type $\left(\alpha_{1}, \alpha_{1}, \alpha_{1}, \alpha_{1}\right)$, and also $\frac{4}{61}=6.6 \%$ of the new pure type $\left(\delta_{1}, \delta_{1}, \delta_{1}, \delta_{1}\right)$.

4. Up to $10^{7}, \frac{2391}{2870}=83.3 \%$ are of mixed type $\left(\alpha_{1}, \alpha_{1}, \alpha_{1}, \delta_{1}\right), \frac{234}{2870}=8.6 \%$ of pure type $\left(\delta_{1}, \delta_{1}, \delta_{1}, \delta_{1}\right), \frac{175}{2870}=6.1 \%$ of pure type $\left(\alpha_{1}, \alpha_{1}, \alpha_{1}, \alpha_{1}\right), \frac{62}{2870}=2.2 \%$ of the new mixed type $\left(\alpha_{1}, \delta_{1}, \delta_{1}, \delta_{1}\right)$, and $\frac{8}{2870}=0.3 \%$ of another new mixed type $\left(\alpha_{1}, \alpha_{1}, \delta_{1}, \delta_{1}\right)$.

Conjecture 8.2. For 3-admissible non-split prime(power) conductors $f=q, f=3$, and $f=9$ with $d \equiv 2(\bmod 3)$ over quadratic fields $K=\mathbb{Q}(\sqrt{d})$ with 3-class rank $\varrho=0$, the relative frequency of nilets slightly decreases from $73 \%$ over $72 \%$ and $71 \%$ to $69 \%$, and the relative frequency of singlets slightly increases from $27 \%$ over $28 \%$ and $29 \%$ to $31 \%$. All singlets are of permanent type $\varepsilon$, showing stagnation. See Theorems 8.3 and 8.6. We conjecture the last percentages for the range $0<d_{L}<10^{7}$ to be close to their asymptotic limit. 
Table 21: Types of doublets with a split prime divisor of regular $f$.

\begin{tabular}{|c||r||rrr|}
\hline $\begin{array}{c}\text { DPF Type } \\
\left(\tau\left(L_{1}\right), \tau\left(L_{2}\right)\right)\end{array}$ & Frequency & $d$ & $f$ & $d_{L}$ \\
\hline$\left(\beta_{2}, \beta_{2}\right)$ & 14 & 23717 & 14 & 4648532 \\
$\left(\beta_{2}, \delta_{2}\right)$ & 1 & 5061 & 39 & 7697781 \\
$\left(\beta_{2}, \varepsilon\right)$ & 14 & 7589 & 14 & 1487444 \\
$(\gamma, \varepsilon)$ & 9 & 1192 & 65 & 5036200 \\
$\left(\delta_{2}, \delta_{2}\right)$ & 71 & 4813 & 14 & 943348 \\
$(\varepsilon, \varepsilon)$ & 327 & 197 & 14 & 38612 \\
\hline Total: & 436 & & & \\
\hline
\end{tabular}

Table 22: Types of doublets with a split prime divisor of irregular $f$.

\begin{tabular}{|c||r||rrr|}
\hline $\begin{array}{c}\text { DPF Type } \\
\left(\tau\left(L_{1}\right), \tau\left(L_{2}\right)\right)\end{array}$ & Frequency & $d$ & $f$ & $d_{L}$ \\
\hline$\left(\beta_{2}, \beta_{2}\right)$ & 34 & 60 & 63 & 238140 \\
$(\varepsilon, \varepsilon)$ & 13 & 204 & 63 & 809676 \\
\hline Total: & 47 & & & \\
\hline
\end{tabular}

\subsection{New features for 3 -class rank $\varrho=1$}

Since ramified extensions $N / K$ for $\varrho=2$ do not occur in the range $0<d_{L}<10^{7}$, it is sufficient to state the following theorem for $\varrho \leq 1$.

Theorem 8.1. Let $K=\mathbb{Q}(\sqrt{d})$ be a real quadratic base field with fundamental discriminant $d$ and 3-class rank $\varrho \leq 1$. Suppose $f=q_{1} \cdot q_{2}$ is a regular 3-admissible conductor for $K$ with two prime divisors $q_{1}$ and $q_{2}$. Then the heterogeneous multiplet $\mathbf{M}\left(K_{f}\right)$ associated with the 3-ring class field $K_{f}$ mod $f$ of $K$ consists of four homogeneous multiplets $\mathbf{M}_{c^{2} d}, c \in$ $\left\{1, q_{1}, q_{2}, f\right\}$ with multiplicities $m(1), m\left(q_{1}\right), m\left(q_{2}\right)$ and $m(f)$. In this order, and in dependence on the 3-ring spaces $V\left(q_{1}\right)$, $V\left(q_{2}\right)$ and $V(f)$, these four multiplicities, forming the signature $\operatorname{sgn}\left(\mathbf{M}\left(K_{f}\right)\right)$ of $\mathbf{M}\left(K_{f}\right)$, are given by

1. $(0,1,1,2), \quad$ if $V(f)=V\left(q_{1}\right)=V\left(q_{2}\right)=V$ (doublet $)$,

2. $(0,1,0,0), \quad$ if $0=V(f)=V\left(q_{2}\right)<V\left(q_{1}\right)=V$,

3. $(0,0,1,0), \quad$ if $0=V(f)=V\left(q_{1}\right)<V\left(q_{2}\right)=V$,

4. $(0,0,0,1), \quad$ if $0=V(f)=V\left(q_{1}\right)=V\left(q_{2}\right)<V$ (singlet),

if $\varrho=0$, and thus 3-Selmer space $V$ is one-dimensional, generated by $\eta \in U_{K}=\langle-1, \eta\rangle$, and by

1. $(1,3,3,6), \quad$ if $V(f)=V\left(q_{1}\right)=V\left(q_{2}\right)=V$ (sextet),

2. $(1,3,0,0), \quad$ if $0<V(f)=V\left(q_{2}\right)<V\left(q_{1}\right)=V$,

3. $(1,0,3,0), \quad$ if $0<V(f)=V\left(q_{1}\right)<V\left(q_{2}\right)=V$,

4. (1, 0, 0,3), if $0<V(f)=V\left(q_{1}\right)=V\left(q_{2}\right)<V$ (triplet),

5. $(1,0,0,0), \quad$ if $0=V(f)<V\left(q_{1}\right) \neq V\left(q_{2}\right)<V$ (nilet with defect $\left.\delta=2\right)$,

if $\varrho=1$, and thus 3-Selmer space $V$ is two-dimensional, generated by $\eta \in U_{K}$ and $\theta \in I \backslash U_{K}$.

Proof. These statements are special cases with $p=3$ of [35, Theorem 5.1].

Remark 8.1. We emphasize that in the situation with $\varrho=0$ a complete heterogeneous nilet with signature $(0,0,0,0)$ is impossible, because there always exists a totally real cubic field L with discriminant $d_{L}$ equal to either $\left(q_{1} q_{2}\right)^{2} d$ or $q_{1}^{2} d$ or $q_{2}^{2} d$.

This is in contrast to the case $\varrho=1$ where a total heterogeneous nilet with signature $(1,0,0,0)$, at least with respect to the ramified components, can occur. In this extreme case of a homogeneous nilet $\mathbf{M}_{f^{2} d}$ with defect $\delta(f)=2$, neither the 
Table 23: Types of triplets with a non-critical split prime conductor $f=\ell$.

\begin{tabular}{|c||r||rrr|}
\hline DPF Type & Frequency & $d$ & $f$ & $d_{L}$ \\
$\left(\tau\left(L_{1}\right), \tau\left(L_{2}\right), \tau\left(L_{3}\right)\right)$ & & & & \\
\hline$\left(\alpha_{2}, \alpha_{2}, \alpha_{2}\right)$ & 10 & 32204 & 7 & 1577996 \\
$\left(\alpha_{2}, \alpha_{2}, \delta_{1}\right)$ & 9 & 2677 & 19 & 966397 \\
$\left(\alpha_{2}, \alpha_{2}, \delta_{2}\right)$ & 23 & 9749 & 13 & 1647581 \\
$\left(\alpha_{2}, \delta_{2}, \delta_{2}\right)$ & 1 & 5477 & 37 & 7498013 \\
$\left(\beta_{1}, \beta_{2}, \beta_{2}\right)$ & 4 & 7244 & 19 & 2615084 \\
$\left(\beta_{1}, \delta_{1}, \delta_{1}\right)$ & 226 & 1765 & 7 & 86485 \\
$\left(\delta_{1}, \delta_{1}, \delta_{1}\right)$ & 23 & 13688 & 13 & 2313272 \\
$\left(\delta_{1}, \delta_{1}, \delta_{2}\right)$ & 1 & 30553 & 13 & 5163457 \\
$\left(\delta_{1}, \delta_{1}, \varepsilon\right)$ & 86 & 3873 & 7 & 189777 \\
$\left(\delta_{1}, \delta_{2}, \delta_{2}\right)$ & 2 & 44641 & 7 & 2187409 \\
$\left(\delta_{2}, \delta_{2}, \delta_{2}\right)$ & 1 & 54469 & 7 & 2668981 \\
\hline Total: & 386 & & & \\
\hline
\end{tabular}

Table 24: Types of triplets with critical split prime power conductor $f=9$.

\begin{tabular}{|c||r||rrr|}
\hline DPF Type & Frequency & $d$ & $f$ & $d_{L}$ \\
$\left(\tau\left(L_{1}\right), \tau\left(L_{2}\right), \tau\left(L_{3}\right)\right)$ & & & & \\
\hline$\left(\alpha_{2}, \alpha_{2}, \alpha_{2}\right)$ & 4 & 14197 & 9 & 1149957 \\
$\left(\alpha_{2}, \alpha_{2}, \delta_{1}\right)$ & 2 & 31069 & 9 & 2516589 \\
$\left(\alpha_{2}, \alpha_{2}, \delta_{2}\right)$ & 5 & 15529 & 9 & 1257849 \\
$\left(\alpha_{2}, \delta_{2}, \delta_{2}\right)$ & 1 & 30904 & 9 & 2503224 \\
$\left(\beta_{1}, \delta_{1}, \delta_{1}\right)$ & 85 & 2917 & 9 & 236277 \\
$\left(\delta_{1}, \delta_{1}, \delta_{1}\right)$ & 6 & 13861 & 9 & 1122741 \\
$\left(\delta_{1}, \delta_{1}, \varepsilon\right)$ & 21 & 15733 & 9 & 1274373 \\
\hline Total: & 124 & & & \\
\hline
\end{tabular}

fundamental unit $\eta$ nor the other generating 3-virtual unit $\theta$ belong to the ring $R_{f}$ modulo $f$ of $K$, i.e. both of them are deficient.

We also point out that Theorem 8.1 is not only valid for $f=q_{1} q_{2}$ with primes $q_{i} \equiv 2(\bmod 3)$ but also for $f=3 q$ with $q_{1}:=3, d \equiv \pm 3(\bmod 9), q_{2}:=q \equiv \pm 1(\bmod 3)$, for $f=9 q$ with $q_{1}:=9$ (the prime power behaves like a prime, formally), $d \equiv \pm 1(\bmod 3), q_{2}:=q \equiv \pm 1(\bmod 3)$, and for $f=q_{1} q_{2}$ with any primes $q_{i} \equiv \pm 1(\bmod 3)$. The statement is independent of the decomposition law of the primes $q_{i}$ in $K$, but it is essential that the conductor is regular, that is, $9 \nmid f$ if $d \equiv 6(\bmod 9)$.

Example 8.1. We explicitly consider the statistical results for $\varrho=0, f=q_{1} q_{2}$ with $q_{1}, q_{2} \equiv 2(\bmod 3)$ in the most extensive range $0<d_{L}<10^{7}$ (Table 13). Since we want to apply probability theory to independent binary properties, we must start with data concerning prime conductors $f=q$.

- Let $f=q \equiv 2(\bmod 3)$ prime. Among 287877 admissible discriminants $q^{2} d$,

$198952(69 \%)$ belong to nilets, realizing the event $V(q)=0$, and

88925 (31\%) belong to singlets, realizing the counter event $V(q)=V$.

- For $f=q_{1} q_{2}$, the four field probability table for independent events yields

$P=0.69^{2} \approx 0.476$ for the event $\left[V\left(q_{1}\right)=0\right.$ and $\left.V\left(q_{2}\right)=0\right]$,

$P=0.69 \cdot 0.31+0.31 \cdot 0.69 \approx 0.214+0.214=0.428$ for the (symmetric) event

$\left[V\left(q_{1}\right)=0\right.$ and $\left.V\left(q_{2}\right)=V\right]$ or $\left[V\left(q_{1}\right)=V\right.$ and $\left.V\left(q_{2}\right)=0\right]$,

$P=0.31^{2} \approx 0.096$ for the event $\left[V\left(q_{1}\right)=V\right.$ and $\left.V\left(q_{2}\right)=V\right]$,

and these theoretical probabilities are indeed compatible with our experimental result that among 6227 admissible discriminants $f^{2} d$,

$2706(43 \% \approx 42.8 \%)$ belong to nilets, $\left[V\left(q_{1}\right)=0 \wedge V\left(q_{2}\right)=V\right] \vee\left[V\left(q_{1}\right)=V \wedge V\left(q_{2}\right)=0\right]$,

$3092(50 \% \approx 47.6 \%)$ belong to singlets, realizing the event $\left[V\left(q_{1}\right)=0\right.$ and $\left.V\left(q_{2}\right)=0\right]$,

$429(7 \% \approx 9.6 \%)$ belong to doublets, realizing the event $\left[V\left(q_{1}\right)=V\right.$ and $\left.V\left(q_{2}\right)=V\right]$. 
Table 25: Types of triplets with a non-split prime (power) conductor.

\begin{tabular}{|c||r||rrr|}
\hline $\begin{array}{c}\text { DPF Type } \\
\left(\tau\left(L_{1}\right), \tau\left(L_{2}\right), \tau\left(L_{3}\right)\right)\end{array}$ & Frequency & $d$ & $f$ & $d_{L}$ \\
\hline$\left(\beta_{1}, \beta_{1}, \beta_{1}\right)$ & 304 & 55885 & 2 & 223540 \\
$\left(\beta_{1}, \beta_{1}, \delta_{1}\right)$ & 160 & 30965 & 2 & 123860 \\
$\left(\beta_{1}, \beta_{1}, \varepsilon\right)$ & 640 & 14397 & 2 & 57588 \\
$\left(\beta_{1}, \delta_{1}, \varepsilon\right)$ & 5 & 417077 & 2 & 1668308 \\
$\left(\beta_{1}, \varepsilon, \varepsilon\right)$ & 10 & 492117 & 2 & 1968468 \\
$\left(\delta_{1}, \delta_{1}, \delta_{1}\right)$ & 2869 & 7053 & 2 & 28212 \\
$\left(\delta_{1}, \delta_{1}, \varepsilon\right)$ & 11 & 486461 & 2 & 1945844 \\
$\left(\delta_{1}, \varepsilon, \varepsilon\right)$ & 35 & 192245 & 2 & 768980 \\
$(\varepsilon, \varepsilon, \varepsilon)$ & 54 & 197445 & 2 & 789780 \\
\hline Total: & 4088 & & & \\
\hline
\end{tabular}

Table 26: Types of triplets with two non-split ramified primes.

\begin{tabular}{|c||r||rrr|}
\hline DPF Type & Frequency & $d$ & $f$ & $d_{L}$ \\
$\left(\tau\left(L_{1}\right), \tau\left(L_{2}\right), \tau\left(L_{3}\right)\right)$ & & & & \\
\hline$\left(\beta_{1}, \beta_{1}, \beta_{1}\right)$ & 221 & 3173 & 10 & 317300 \\
$\left(\beta_{1}, \beta_{1}, \gamma\right)$ & 5 & 63917 & 10 & 6391700 \\
$\left(\beta_{1}, \gamma, \gamma\right)$ & 6 & 82397 & 10 & 8239700 \\
$(\gamma, \gamma, \gamma)$ & 6 & 9413 & 22 & 4555892 \\
$(\gamma, \gamma, \varepsilon)$ & 3 & 64677 & 10 & 6467700 \\
$(\varepsilon, \varepsilon, \varepsilon)$ & 56 & 9293 & 10 & 929300 \\
\hline Total: & 297 & & & \\
\hline
\end{tabular}

Since almost identical probabilities as for the conductors $f=q_{1} q_{2}$ with $q_{1}, q_{2} \equiv 2(\bmod 3)$ arise for all the other regular conductors with two prime divisors in Theorem 8.1, mentioned explicitly at the end of Remark 8.1, we are convinced of the following experimental hypothesis.

Conjecture 8.3. (Probability for $m \in\{0,1,2\}$ when $\varrho=0$ )

The probabilities $P$ for the occurrence of various multiplets $\left(L_{1}, \ldots, L_{m}\right)$ of totally real cubic fields $L_{i}$ among sets of 3admissible pairs $(f, d)$ of regular conductors $f$ and quadratic fundamental discriminants $d>0$ with $\varrho=0$ are approximately given as follows:

1. $P \approx 31 \%$ for a singlet, and $P \approx 69 \%$ for a nilet, when $f=q$,

2. $P \approx 7 \%$ for a doublet, $P \approx 50 \%$ for a singlet, and $P \approx 43 \%$ for a nilet, when $f=q_{1} q_{2}$.

Example 8.2. Now we present new features for $\varrho=1, f=q_{1} q_{2}$ with $q_{1}, q_{2} \equiv 2(\bmod 3)$ in the most extensive range $0<d_{L}<10^{7}$ (Table 14). Again, we must begin with prime conductors $f=q$.

- Let $f=q \equiv 2(\bmod 3)$ prime. Among 41541 admissible discriminants $q^{2} d$, $38302(92.2 \%)$ belong to nilets, realizing the event $V(q)<V$, and 3239 (7.8\%) belong to triplets, realizing the counter event $V(q)=V$.

- For $f=q_{1} q_{2}$, the four field probability table for independent events yields

$P=0.922^{2} \approx 0.850$ for the event $\left[V\left(q_{1}\right)<V\right.$ and $\left.V\left(q_{2}\right)<V\right]$,

$P=0.922 \cdot 0.078+0.078 \cdot 0.922 \approx 0.072+0.072=0.144$ for the (symmetric) event

$\left[V\left(q_{1}\right)<V\right.$ and $\left.V\left(q_{2}\right)=V\right]$ or $\left[V\left(q_{1}\right)=V\right.$ and $\left.V\left(q_{2}\right)<V\right]$,

$P=0.078^{2} \approx 0.006$ for the event $\left[V\left(q_{1}\right)=V\right.$ and $\left.V\left(q_{2}\right)=V\right]$,

but these theoretical probabilities are not immediately compatible with our experimental result that among 649 admissible discriminants $f^{2} d$,

$534(82.3 \%)$ belong to nilets,

$115(17.7 \%)$ belong to triplets,

$0(0 \% \approx 0.6 \%)$ belong to sextets, realizing the event $\left[V\left(q_{1}\right)=V\right.$ and $\left.V\left(q_{2}\right)=V\right]$.

Only the case of sextets is compatible, in the sense that it has simply not occurred yet in this range. At this point, 
Table 27: Types of triplets with non-split and split ramified primes.

\begin{tabular}{|c||r||rrr|}
\hline $\begin{array}{c}\text { DPF Type } \\
\left(\tau\left(L_{1}\right), \tau\left(L_{2}\right), \tau\left(L_{3}\right)\right)\end{array}$ & Frequency & $d$ & $f$ & $d_{L}$ \\
\hline$\left(\alpha_{2}, \alpha_{2}, \alpha_{2}\right)$ & 5 & 6997 & 14 & 1371412 \\
$\left(\beta_{1}, \beta_{1}, \beta_{1}\right)$ & 83 & 1101 & 14 & 215796 \\
$\left(\beta_{1}, \beta_{1}, \beta_{2}\right)$ & 1 & 21324 & 21 & 9403884 \\
$\left(\beta_{1}, \beta_{2}, \beta_{2}\right)$ & 3 & 29317 & 14 & 5746132 \\
$\left(\beta_{2}, \beta_{2}, \beta_{2}\right)$ & 1 & 18661 & 18 & 6046164 \\
$\left(\beta_{1}, \beta_{1}, \gamma\right)$ & 2 & 469 & 62 & 1802836 \\
$\left(\delta_{2}, \delta_{2}, \delta_{2}\right)$ & 4 & 24621 & 14 & 4825716 \\
$(\varepsilon, \varepsilon, \varepsilon)$ & 11 & 10733 & 14 & 2103668 \\
\hline Total: & 110 & & & \\
\hline
\end{tabular}

Table 28: Smallest occurrences of various singlets.

\begin{tabular}{|c||rrr|}
\hline $\begin{array}{c}\text { DPF Type } \\
(\tau(L))\end{array}$ & $d$ & $f$ & $d_{L}$ \\
\hline$(\varepsilon)$ & 37 & 2 & 148 \\
$\left(\delta_{1}\right)$ & 229 & 1 & 229 \\
$(\gamma)$ & 21 & 6 & 756 \\
$\left(\delta_{2}\right)$ & 53 & 7 & 2597 \\
$\left(\beta_{2}\right)$ & 29 & 14 & 5684 \\
$\left(\alpha_{3}\right)$ & 37 & 63 & 146853 \\
\hline
\end{tabular}

a new phenomenon appears: the possibility of elevated defect $\delta(f)=2$, when $0=V(f)<V\left(q_{1}\right) \neq V\left(q_{2}\right)<V$. We have to split the event $\left[V\left(q_{1}\right)<V \wedge V\left(q_{2}\right)<V\right]$, with theoretical probability $85.0 \%$, into two cases, a triplet for $0<V(f)=V\left(q_{1}\right)=V\left(q_{2}\right)<V$ with experimental probability $17.7 \%$, and a nilet for $0=V(f)<V\left(q_{1}\right) \neq V\left(q_{2}\right)<V$ with unknown probability, which can now be calculated as $85.0 \%-17.7 \%=67.3 \%$, an astonishingly high value. Eventually, the sum of the probabilities for nilets with $\delta=1$ and nilets with $\delta=2$, that is, $14.4 \%+67.3 \%=81.7 \% \approx 82.3 \%$ agrees with the experimental probability for all nilets, indeed.

Conjecture 8.4. (Probability for $m \in\{0,3,6\}$ when $\varrho=1$ )

The probabilities $P$ for the occurrence of various multiplets $\left(L_{1}, \ldots, L_{m}\right)$ of totally real cubic fields $L_{i}$ among sets of 3 admissible pairs $(f, d)$ of regular conductors $f$ and quadratic fundamental discriminants $d>0$ with $\varrho=1$ are approximately given as follows:

1. $P \approx 8 \%$ for a triplet, and $P \approx 92 \%$ for a nilet, when $f=q$,

2. $P \approx 1 \%$ for a sextet, $P \approx 17 \%$ for a triplet, and $P \approx 82 \%$ for a nilet, when $f=q_{1} q_{2}$. Among the $82 \%$ for a nilet, there are $18 \%$ nilets with $\delta=1$ and $82 \%$ nilets with $\delta=2$.

Example 8.3. It is illuminating to give particular realizations of the various multiplets in Theorem 8.1. Let $q_{1}=2$ and $q_{2}=5$ and consider the composite conductor $f=q_{1} q_{2}=10$.

- Among quadratic fundamental discriminants $d$ with $\varrho=0$, there are four $d \in\{5, \mathbf{1 3}, 21,29\}$ which give rise to nilets $\mathbf{M}_{4 d}=\emptyset$ before we find a singlet with conductor 2 for $d=37, d_{L}=148$, and there are eight $d \in\{8,12, \mathbf{1 3}, 17,28,33,37,53\}$ giving rise to nilets $\mathbf{M}_{25 d}=\emptyset$ until a singlet with conductor 5 occurs for $d=57, d_{L}=1425$. The consequence of the simultaneous nilets $\mathbf{M}_{4 d}=\mathbf{M}_{25 d}=\emptyset$ for $d=\mathbf{1 3}$ is the existence of a singlet with conductor $f=10$ and $d_{L}=1300$ in spite of positive defect $\delta(10)=1$. A nilet $\mathbf{M}_{100 d}=\emptyset$ with conductor $f=10$ arises for $d=37$, because $\mathbf{M}_{4 d}$ is a singlet and $\mathbf{M}_{25 d}=\emptyset$ is a nilet. We have to wait for the sixteenth discriminant $d$ for which $f=10$ is admissible in order to encounter the first doublet $\mathbf{M}_{100 d}$ for $d=373, d_{L}=37300$ with vanishing defect $\delta(10)=0$.

- Among quadratic fundamental discriminants $d$ with $\varrho=1$, the probability $P \approx 92 \%>69 \%$ for a nilet with prime conductor is higher, and thus we have to skip 56 discriminants, commencing with $d \in\{229,469,733, \ldots\}$ until the first triplet $\mathbf{M}_{4 d}$ with conductor 2 occurs for $d=7053, d_{L}=28212$. Similarly, we must overleap 7 discriminants, beginning with $d \in\{257,473,568,697, \mathbf{7 3 3}, \ldots\}$ before we find a triplet $\mathbf{M}_{25 d}$ with conductor 5 for $d=1257, d_{L}=31425$. Now the new feature of elevated defect $\delta=2$ for positive 3-class rank sets in: The consequence of the simultaneous nilets $\mathbf{M}_{4 d}=\mathbf{M}_{25 d}=\emptyset$ for 
$d=733$ is not at all a triplet, but rather a nilet $\mathbf{M}_{100 d}=\emptyset$ with $f=10$, because the ring spaces $V(2)$ and $V(5)$ have trivial meet, whence $\delta(10)=2$. This phenomenon continues for further six discriminants starting with $d \in\{1373,1957,2213\}$ until $V(10)=V(2)=V(5)$ coincide for $d=3173, d_{L}=317300$, giving rise to the first triplet $\mathbf{M}_{100 d}$. Even later, the first nilet with moderate defect $\delta(10)=1$ (it is the 24 th in the series of nilets) occurs for $d=7053$, since $\mathbf{M}_{4 d}$ is a triplet and $\mathbf{M}_{25 d}=\emptyset$ is a nilet. This ostensively shows the dominant role of the $82 \%$ nilets $\mathbf{M}_{100 d}=\emptyset$ with $\delta=2$ as opposed to the $18 \%$ with $\delta=1$, according to Conjecture 8.4.

\subsection{Unramified extensions}

The unique conductor without prime divisors is $f=1$. It is 3-admissible for any quadratic fundamental discriminant $d$.

Among the 3039653 quadratic fundamental discriminants in the range $0<d<10^{7}$, there are 2623325 , respectively 413458 , respectively 2870 , which give rise to real quadratic number fields $K=\mathbb{Q}(\sqrt{d})$ with 3-class rank $\varrho=\varrho_{3}(K)=0$, respectively 1 , respectively 2 . According to the multiplicity formula $m=m_{3}(K, 1)=\frac{3^{e}-1}{3-1}$, there exist 0 , respectively 413458 , respectively 11480 , totally real cubic fields $L$ with discriminant $d_{L}=f^{2} \cdot d=1^{2} \cdot d=d$, occurring in nilets, respectively singlets, respectively quartets. The associated normal closure $N$ of each of these non-Galois cubic fields $L$ is unramified over its unique quadratic subfield $K$.

Example 8.4. The smallest discriminant with $\varrho=0$ is $d=5$. Although it is an actual quadratic fundamental discriminant, it is only a formal cubic discriminant belonging to a nilet. The minimal discriminants $d=229$, respectively $d=32009$, with $\varrho=1$, respectively $\varrho=2$, are both, fundamental discriminants of real quadratic fields and actual discriminants of totally real cubic fields belonging to a singlet, respectively quartet. The latter two discriminants are contained in the table of Angell with $0<d_{L}<10^{5}$ already.

In the sequel, we briefly speak about the type $\tau(N)=\tau(L) \in\left\{\alpha_{1}, \alpha_{2}, \alpha_{3}, \beta_{1}, \beta_{2}, \gamma, \delta_{1}, \delta_{2}, \varepsilon\right\}$ of a totally real $S_{3}$-field $N$, respectively its three conjugate cubic subfields $L$, when we specify the differential principal factorization type of $N$, respectively $L$.

Theorem 8.2. Let L be a non-Galois totally real cubic field whose normal closure $N$ is unramified over its quadratic subfield $K$, with conductor $f=1$.

1. If the 3-class group $\mathrm{Cl}_{3}(K)$ is non-trivial cyclic, then $L$ must be of type $\tau(L)=\delta_{1}$.

2. If $K$ has 3 -class rank $\varrho \geq 2$, then two types $\tau(L) \in\left\{\alpha_{1}, \delta_{1}\right\}$ are possible for $L$.

Proof. See Theorem 8.6 (1) for item (1), and Theorem 3.6 with $t=s=0$ and thus $A=R=0$ for item (2).

\subsection{Conductors with a single prime divisor}

Example 8.5. It is conspicuous, that the range $0<d<10^{7}$ contains an abundance of 197 nilets with formal cubic discriminants $f^{2} \cdot d$ such that the conductor $f=q$ is a prime $q \equiv 2(\bmod 3)$ and the fundamental discriminant $d$ belongs to a real quadratic field $K$ with 3-class rank $\varrho=2$. The smallest values of $d$ occurring among these 197 cases are 32009,42817 , 62 501. However, the associated formal cubic discriminants appear in reverse order $250004=2^{2} \cdot 62501,1070425=5^{2} \cdot 42817$, $3873089=11^{2} \cdot 32009$, due to the conductors which increase in the opposite direction. In particular, the smallest formal cubic discriminant 250004 lies in the range $0<d<5 \cdot 10^{5}$ of Ennola and Turunen already. Actual nonets $(m=9)$ of cubic fields with these discriminants do not exist. According to a private communication by Karim Belabas on 31 January 2002, the discriminant $18251060=2^{2} \cdot 4562765$ in Theorem 9.4 is not only minimal with a ramified $(f=2)$ component of type $\alpha_{1}$, as required for the proof of the Scholz Conjecture, but even the minimal discriminant of totally real cubic nonets at all (see http://www. algebra.at/KarimDan5.htm).

Theorem 8.3. Let $L$ be a totally real cubic field whose normal closure $N$ is ramified over its quadratic subfield $K$ with $\varrho=0$ and conductor $f$ divisible by a single non-split prime,

1. either $f=q$ a prime $q \equiv 2(\bmod 3)$, inert in $K$,

2. or $f=3$ with $d \equiv 3(\bmod 9)$ or $d \equiv 6(\bmod 9)$

3. or $f=9$ with $d \equiv 6(\bmod 9)$

4. or $f=9$ with $d \equiv 2(\bmod 3)$. 
In the second and third case, 3 ramifies in $K$, in the fourth case, 3 remains inert in $K$.

Then $L$ must necessarily be of type $\tau(L)=\varepsilon$.

Proof. See Theorem 8.6 (2).

\subsection{General conditions for differential principal factorizations}

The nine possible types $\tau(L)=\tau(N) \in\left\{\alpha_{1}, \alpha_{2}, \alpha_{3}, \beta_{1}, \beta_{2}, \gamma, \delta_{1}, \delta_{1}, \varepsilon\right\}$ of differential principal factorizations of a non-cyclic totally real cubic field $L$, more precisely of the totally real Galois closure $N$ of $L$, are defined with the aid of three invariants $A, R$ and $C$ which are $\mathbb{F}_{3}$-dimensions of canonical subspaces of the vector space $\mathcal{P}_{N / K} / \mathcal{P}_{K}$ of primitive ambiguous principal ideals of $N$ over its quadratic subfield $K$.

The most restrictive necessary conditions are imposed by the three types $\alpha_{1}, \alpha_{3}, \gamma$ which are characterized by twodimensional subspaces.

Theorem 8.4. (Necessary conditions for two-dimensional subspaces)

1. For type $\gamma$ with two-dimensional absolute principal factorization $A=2$, the conductor $f$ must have at least two prime divisors, $t \geq 2$.

2. For type $\alpha_{3}$ with two-dimensional relative principal factorization $R=2$, the conductor $f$ must have at least two prime divisors which split in $K, s \geq 2$ (and a fortiori $t \geq 2$ ).

3. For type $\alpha_{1}$ with two-dimensional capitulation $C=2$, the 3-class rank $\varrho$ of $K$ must be at least two (independently of the conductor $f \geq 1$ ).

Proof. We make use of the fundamental inequalities in Corollary 3.2:

$$
0 \leq A \leq \min (n+s, 2), \quad 0 \leq R \leq \min (s, 2), \quad 0 \leq C \leq \min (\varrho, 2) .
$$

1. Type $\gamma \Longleftrightarrow A=2 \Longrightarrow \min (n+s, 2)=2$, i.e. $t=n+s \geq 2$.

2. Type $\alpha_{3} \Longleftrightarrow R=2 \Longrightarrow \min (s, 2)=2$, i.e. $s \geq 2$, and thus $t=n+s \geq s \geq 2$.

3. Type $\alpha_{1} \Longleftrightarrow C=2 \Longrightarrow \min (\varrho, 2)=2$, i.e. $\varrho \geq 2$.

Looser necessary conditions are required for non-trivial subspaces.

Theorem 8.5. (Necessary conditions for one-dimensional subspaces)

1. For the types $\beta_{1}, \beta_{2}, \varepsilon$ with one-dimensional absolute principal factorization $A=1$, the conductor $f$ must have at least one prime divisor, $t \geq 1$.

2. For the types $\alpha_{2}, \beta_{2}, \delta_{2}$ with one-dimensional relative principal factorization $R=1$, the conductor $f$ must have at least one prime divisor which splits in $K, s \geq 1$ (thus $t \geq 1$ ).

3. For the types $\alpha_{2}, \beta_{1}, \delta_{1}$ with one-dimensional capitulation $C=1$, the 3 -class rank $\varrho$ of $K$ must be at least one.

For each of the types $\alpha_{2}, \beta_{1}, \beta_{2}$, two suitable among these conditions may be combined.

Proof. According to the definitions of DPF types and the fundamental inequalities in Corollary 3.2:

1. Type $\beta_{1}, \beta_{2}, \varepsilon \Longleftrightarrow A=1 \Longrightarrow \min (n+s, 2) \geq 1$, i.e. $t=n+s \geq 1$.

2. Type $\alpha_{2}, \beta_{2}, \delta_{2} \Longleftrightarrow R=1 \Longrightarrow \min (s, 2) \geq 1$, i.e. $s \geq 1$, and thus $t=n+s \geq s \geq 1$.

3. Type $\alpha_{2}, \beta_{1}, \delta_{1} \Longleftrightarrow C=1 \Longrightarrow \min (\varrho, 2) \geq 1$, i.e. $\varrho \geq 1$.

Due to the fact that the occurrence of absolute principal factorizations is usually unpredictable as soon as the conductor $f>1$ has at least one prime divisor, $t \geq 1$, there a only very few sufficient conditions for DPF types. Only two types can be enforced unambiguously.

Theorem 8.6. (Sufficient conditions for types $\delta_{1}$ and $\varepsilon$ )

1. If $N / K$ is unramified with conductor $f=1$ and $K$ has 3-class rank $\varrho=1$, then $\tau(N)=\delta_{1}$. 
2. If the conductor $f$ of $N / K$ has precisely one prime divisor which does not split in $K$ and the class number of $K$ is not divisible by 3 , then $\tau(N)=\varepsilon$.

In both cases, there exists a unit $H \in U_{N}$ such that $\eta=N_{N / K}(H)$ is a fundamental unit of $K$.

Proof. According to the fundamental inequalities in Corollary 3.2 and the fundamental equation in Corollary 3.1, we have:

1. $t=0, \varrho=1 \Longrightarrow A \leq \min (n+s, 2)=\min (t, 2)=0, s \leq t=0, R \leq \min (s, 2)=0, C \leq \min (\varrho, 2)=1$, but on the other hand $C=0+0+C=A+R+C=U+1 \geq 1 \Longrightarrow A=R=0, C=1 \Longleftrightarrow$ Type $\delta_{1}$.

2. $t=1, s=0, \varrho=0 \Longrightarrow A \leq \min (n+s, 2)=\min (t, 2)=1, R \leq \min (s, 2)=0$, and $C \leq \min (\varrho, 2)=0$, but on the other hand $A=A+0+0=A+R+C=U+1 \geq 1 \Longrightarrow A=1, R=C=0 \Longleftrightarrow$ Type $\varepsilon$.

In both cases, we obtain $U=0$ as a byproduct, i.e. $N_{N / K}\left(U_{N}\right)=U_{K}$.

\section{Complete verification of the Scholz conjecture}

Let $L$ be a non-cyclic totally real cubic field. Then $L$ is non-Galois over the rational number field $\mathbb{Q}$ with two conjugate fields $L^{\prime}$ and $L^{\prime \prime}$. The Galois closure $N$ of $L$ is a totally real dihedral field of degree 6, i.e. an $S_{3}$-field, which contains a unique real quadratic field $K$, as illustrated in Figure 5.

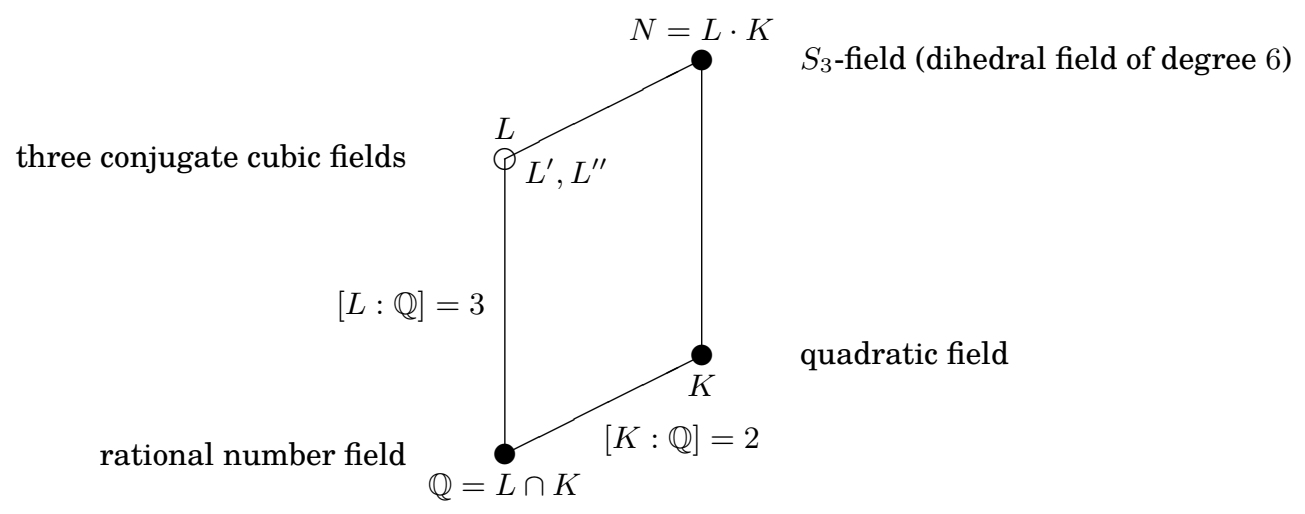

Figure 5: Hasse subfield diagram of the normal closure $N / \mathbb{Q}$ of $L$.

In 1930, Hasse [14] determined the discriminants $d_{L}$ of $L$ [14, pp. 567 (1) and 575] and $d_{N}$ of $N$ [14, p. 566 (2)], in dependence on the discriminant $d=d_{K}$ of $K$ and on the class field theoretic conductor $f=f_{N / K}$ of the cyclic cubic, and thus Abelian, relative extension $N / K$ :

$$
d_{L}=f^{2} \cdot d, \quad \text { and } \quad d_{N}=f^{4} \cdot d^{3} .
$$

Three years later, Scholz [39, p. 216] determined the relation

$$
h_{N}=\frac{a}{9} \cdot h_{L}^{2} \cdot h_{K}
$$

between the class numbers of the fields $N, L$ and $K$, in dependence on the index of subfield units, $a=\left(U_{N}: U_{0}\right)=3^{E}$, where $U_{0}=\left\langle U_{K}, U_{L}, U_{L^{\prime}}, U_{L^{\prime \prime}}\right\rangle$ and $E \in\{0,1,2\}$.

Note that $E=0$, respectively $a=1$, is the distinguished situation where the unit group $U_{N}$ of the normal field $N$ is entirely generated by all proper subfield units, that is, $U_{N}=U_{0}$.

Scholz was able to give explicit numerical examples [39, p. 216] for $E=1$ (e.g. $d_{L}=229$ ), and $E=2$ (e.g. $d_{L}=148$ ), but not for $E=0$, and he formulated the following hypothesis.

Conjecture 9.1. (The Scholz Conjecture, 1933, illustrated in Figure 6)

There should exist non-Galois totally real cubic fields L whose Galois closure $N$ is either

1. unramified, with conductor $f=1$, over some real quadratic field $K$ with 3-class rank $\varrho_{3}(K)=2$ whose complete 3elementary class group capitulates in $N$ such that $U_{N}=U_{0}$ (in the terminology of $S c h o l z, N$ is an absolute class field over K) [39, p. 219], or 

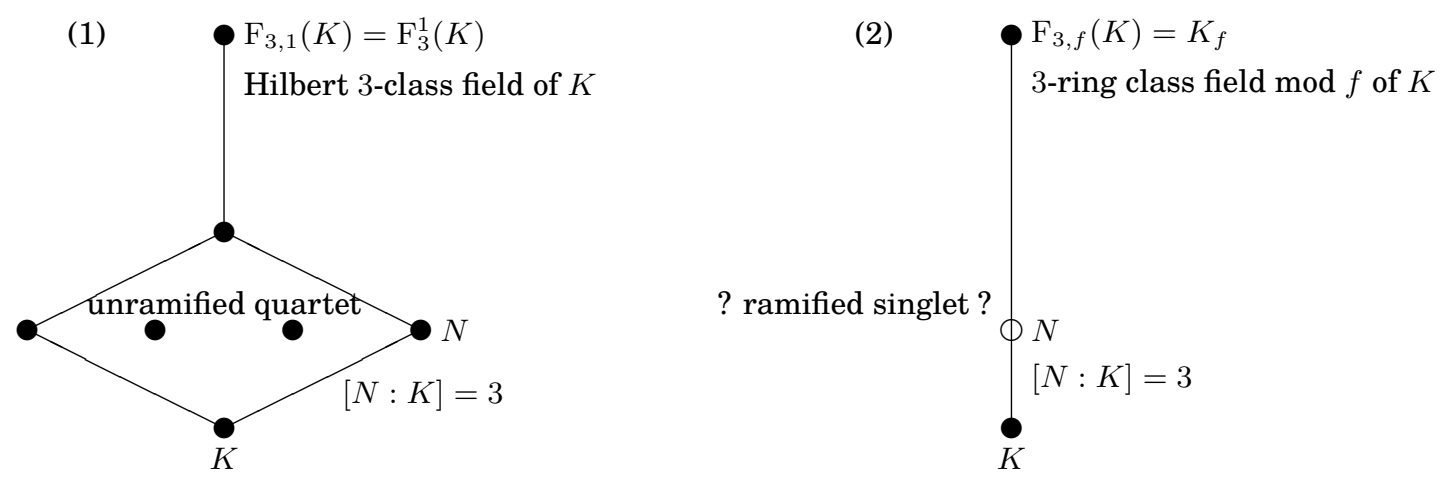

Figure 6: Hilbert and ring class fields over $K$.

2. ramified, with conductor $f>1$, over some real quadratic field $K$ such that $U_{N}=U_{0}$ (here, Scholz calls $N$ a ring class field over K, by abuse of language) [39, p. 221].

We point out that, in the unramified situation $f=1, d_{L}=d$ is a quadratic fundamental discriminant, and $d_{N}=d^{3}$ is a perfect cube, according to Formula (15). In this unramified case, the verification of Conjecture 9.1 can be obtained from a more general theorem, since any real quadratic field $K$ with 3-class rank $\varrho_{3}(K)=2$ possesses a multiplet of four unramified cyclic cubic extensions $N_{1}, \ldots, N_{4}$, that is a quartet of absolutely dihedral fields of degree 6 [29] with non-Galois totally real cubic subfields $L_{1}, \ldots, L_{4}$, each of them selected among three conjugate fields.

For such a quartet, Chang and Foote [8] introduced the concept of the capitulation number $0 \leq \nu(K) \leq 4$, defined as the number of those members of the quartet in which the complete 3-elementary class group of $K$ capitulates. For this number $\nu(K)$, the following theorem holds.

Theorem 9.1. For each value $0 \leq \nu \leq 4$, there exists a real quadratic field $K$ with 3-class rank $\varrho_{3}(K)=2$ and capitulation number $\nu(K)=\nu$. It is even possible to restrict the claim to fields with elementary 3-class group of type $\mathrm{Cl}_{3}(K) \simeq C_{3} \times C_{3}$.

Proof. From the viewpoint of finite $p$-group theory, this theorem is a proven statement about the possible transfer kernel types of finite metabelian 3-groups $G$ with abelianization $G / G^{\prime} \simeq(3,3)$ applied to the second 3-class group $G:=$ $\operatorname{Gal}\left(F_{3}^{2}(K) / K\right)$ of $K$ [29]. However, it is easier to give explicit numerical paradigms for each value of $\nu(K)$. We have the following minimal occurrences:

$\nu(K)=4$ for $d_{K}=62501$,

$\nu(K)=3$ for $d_{K}=32009$,

$\nu(K)=2$ for $d_{K}=710652$,

$\nu(K)=1$ for $d_{K}=534824$,

$\nu(K)=0$ for $d_{K}=214712$,

which have been computed by ourselves in [29]. The existence of these cases completes the proof.

Remark 9.1. We have the priority of discovering the first examples of real quadratic fields $K$ with $\nu(K) \in\{0,1,2\}$ in [29]. However, the first examples of real quadratic fields $K$ with $\nu(K) \in\{3,4\}$ are due to Heider and Schmithals [17], who performed a mainframe computation on the CDC Cyber of the University at Cologne, and thus the following corollary is proven since 1982 already.

Corollary 9.1. (Verification of Conjecture 9.1, (1) for unramified extensions; see Figure 7)

There exist non-Galois totally real cubic fields L whose Galois closure $N$ is unramified, with conductor $f=1$, over a real quadratic field $K$ with 3-class rank $\varrho_{3}(K)=2$ whose complete 3-elementary class group capitulates in $N$, and which therefore has $U_{N}=U_{0}$. The minimal discriminant of such a field $L$ is $d_{L}=32009$ (discovered in [17], actually, the first three members of this quartet with DPF type $\left(\alpha_{1}, \alpha_{1}, \alpha_{1}, \delta_{1}\right)$ in Table 5 satisfy the relation $\left.U_{N}=U_{0}\right)$.

Proof. It suffices to take a real quadratic field $K$ with $1 \leq \nu(K) \leq 4$ in Theorem 9.1. In view of the minimal discriminant, we select $\nu(K)=3$ and obtain $U_{N}=U_{0}$ for $d_{L}=d_{K}=32009$.

Concerning the ramified situation $f>1$ in Conjecture 9.1 (2), Scholz does not explicitly impose any conditions on the underlying real quadratic field $K$. We suppose that he also tacitly assumed a real quadratic field $K$ with 3-class rank 


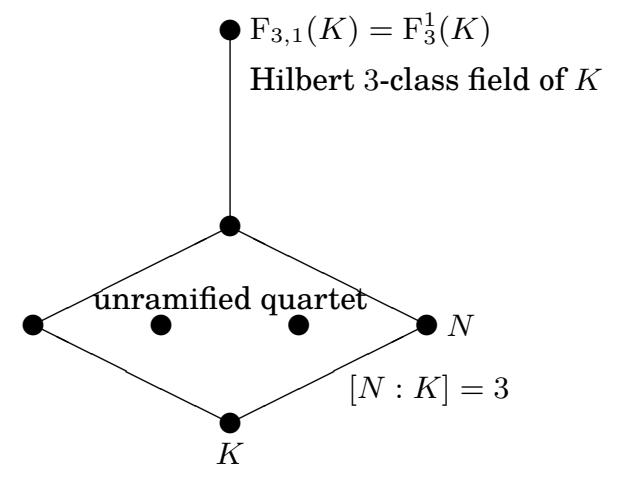

Figure 7: Hilbert class field over $K$.

$\varrho_{3}(K)=2$. However, more recent extensions of the theory of dihedral fields by means of differential principal factorizations and Galois cohomology, two concepts which we have expanded thoroughly in the preparatory Sections 3.2, 3.4, and 3.5, revealed that for $U_{N}=U_{0}$ no constraints on the $p$-class rank $\varrho_{p}(K)$ are required. In 1975, Nicole Moser [36] used the Galois cohomology $\hat{\mathrm{H}}^{0}\left(G, U_{N}\right) \simeq U_{K} / \mathrm{N}_{N / K}\left(U_{N}\right)$ of the unit group $U_{N}$ of the normal closure $N$ as a module over $G=\operatorname{Gal}(N / K)$ to establish a fine structure with five possible types $\alpha, \beta, \gamma, \delta, \varepsilon$ on the coarse classification by three possible values of the index of subfield units:

$\left(U_{N}: U_{0}\right)=1 \Longleftrightarrow$ type $\alpha$ with $\left(U_{K}: \mathrm{N}_{N / K}\left(U_{N}\right)\right)=3$,

$\left(U_{N}: U_{0}\right)=3 \Longleftrightarrow$ type $\beta$ with $\left(U_{K}: \mathrm{N}_{N / K}\left(U_{N}\right)\right)=3$ or type $\delta$ with $\left(U_{K}: \mathrm{N}_{N / K}\left(U_{N}\right)\right)=1$,

$\left(U_{N}: U_{0}\right)=9 \Longleftrightarrow$ type $\gamma$ with $\left(U_{K}: \mathrm{N}_{N / K}\left(U_{N}\right)\right)=3$ or type $\varepsilon$ with $\left(U_{K}: \mathrm{N}_{N / K}\left(U_{N}\right)\right)=1$.

Thus, Moser's refinement does not illuminate the situation $U_{N}=U_{0}$ ( $\Longleftrightarrow$ type $\alpha$ ) of Scholz's conjecture more closely. Meanwhile, Barrucand and Cohn [4] had coined the concept of (differential) principal factorization ((D)PF) for pure cubic fields. In 1991, we generalized the theory of DPFs for dihedral fields of both signatures [26], and we obtained a hyperfine structure by splitting Moser's types further according to the $\mathbb{F}_{p}$-dimensions $C$ of the capitulation $\operatorname{kernel} \operatorname{ker}\left(T_{K, N}\right)$ and $R$ of the space of relative DPFs of $N / K$, which we recalled in the preparatory section 3.7. In particular, type $\alpha$ with $U_{N}=U_{0}$ splits into three subtypes:

type $\alpha_{1} \Longleftrightarrow C=2, R=0$, which implies $\varrho_{p}(K) \geq 2$, type $\alpha_{2} \Longleftrightarrow C=1, R=1$, which implies $\varrho_{p}(K) \geq 1$ and a split prime divisor of $f(s \geq 1)$, type $\alpha_{3} \Longleftrightarrow C=0, R=2$, which is compatible with any $\varrho_{p}(K) \geq 0$, but requires $s \geq 2$.

Consequently, we were led to the following refinement of Conjecture 9.1, (2).

Conjecture 9.2. (Conjecture of D. C. Mayer, 1991)

Non-Galois totally real cubic fields $L$ whose Galois closure $N$ is ramified, with conductor $f>1$, over some real quadratic field $K$, and is of type $\alpha$, with $U_{N}=U_{0}$, should exist for each of the following three situations:

(2.1) type $\alpha_{1}$ with $\operatorname{dim}_{\mathbb{F}_{3}}\left(\operatorname{ker}\left(T_{K, N}\right)\right)=2$ and $\varrho_{3}(K)=2, s=0$,

(2.2) type $\alpha_{2}$ with $\operatorname{dim}_{\mathbb{F}_{3}}\left(\operatorname{ker}\left(T_{K, N}\right)\right)=1$ and $\varrho_{3}(K)=1, s=1$,

(2.3) type $\alpha_{3}$ with $\operatorname{dim}_{\mathbb{F}_{3}}\left(\operatorname{ker}\left(T_{K, N}\right)\right)=0$ and $\varrho_{3}(K)=0, s=2$,

where $T_{K, N}: \mathrm{Cl}_{3}(K) \rightarrow \mathrm{Cl}_{3}(N), \mathfrak{a} \cdot \mathcal{P}_{K} \mapsto\left(\mathfrak{a} \mathcal{O}_{N}\right) \cdot \mathcal{P}_{N}$, denotes the transfer homomorphism of 3-classes from $K$ to $N$, and $s$ counts the prime divisors of the conductor $f$ which split in $K$.

Theorem 9.2. (Verification of Conjecture 9.2, (2.3), and Conjecture 9.1, (2); see Figure 8)

There exist non-Galois totally real cubic fields $L$ whose Galois closure $N$ is ramified, with conductor $f>1$ divisible by two prime divisors which split in $K$, i.e. $s=2$, over a real quadratic field $K$ with 3-class rank $\varrho_{3}(K)=0$, without capitulation in $N$, and such that $U_{N}=U_{0}$. The minimal discriminant of such a field $L$ is $d_{L}=146853=(7 \cdot 9)^{2} \cdot 37$ (which forms a singlet [27]).

Proof. This was proved in the numerical supplement [27] of our paper [26] by computing a gapless list of all 10015 totally real cubic fields $L$ with discriminants $d_{L}<200000$ on the AMDAHL mainframe of the University of Manitoba. There occurred the minimal discriminant $d_{L}=146853=f^{2} \cdot d_{K}$ with $d_{K}=37$ and conductor $f=63=3^{2} \cdot 7$ divisible by two primes which both split in $K$, i.e. $s=2$. This is a necessary requirement for a two-dimensional relative principal factorization with $R=2$ and is unique up to $d_{L}<200000$. (The next is $d_{L}=240149$ with $f=7 \cdot 13$.) There is only a single field $L$ with this discriminant $d_{L}=146853$ (forming a singlet). 


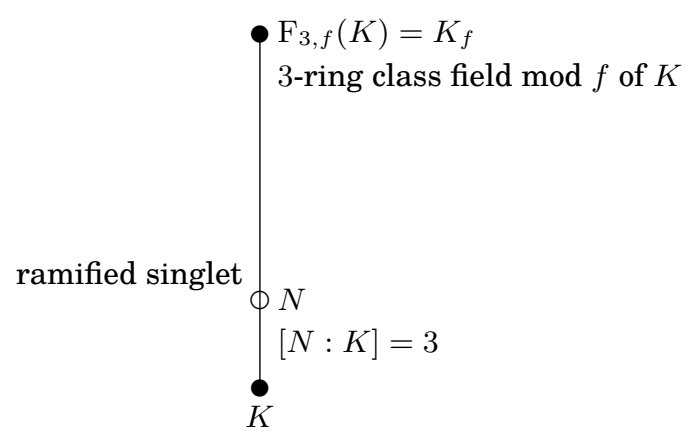

Figure 8: Ring class field modulo $f=63=3^{2} \cdot 7$ over $K$.

Our discovery of the truth of Theorem 9.2 with the aid of the list [27] was a random hit without explicit intention to find a verification of Scholz's conjecture. Unfortunately, [27] does not contain examples of the unique missing DPF type $\alpha_{2}$. It required more than 25 years until we focused on an attack against this lack of information. In contrast to the techniques of [27], we did not use the Voronoi algorithm [40] after cumbersome preparation of generating polynomials for totally real cubic fields, but rather Fieker's class field theory routines of Magma [6, 7, 11,25] for a direct generation of the fields as subfields of 3-ray class fields modulo conductors $f>1$.

Theorem 9.3. (Verification of Conjecture 9.2, (2.2), and Conjecture 9.1, (2); see Figure 9)

There exist non-Galois totally real cubic fields $L$ whose Galois closure $N$ is ramified, with conductor $f>1$ divisible by a single prime divisor that splits in $K$, i.e. $s=1$, over a real quadratic field $K$ with 3 -class rank $\varrho_{3}(K)=1$, with onedimensional capitulation of the elementary 3-class group in $N$, and such that $U_{N}=U_{0}$. The minimal discriminant of such a field $L$ is $d_{L}=\mathbf{9 6 6 3 9 7}=19^{2} \cdot 2677$ (the first two fields of a triplet $\left(\alpha_{2}, \alpha_{2}, \delta_{1}\right)$, discovered 19 November 2017).

Proof. The proof is conducted in the following Section 9.1.

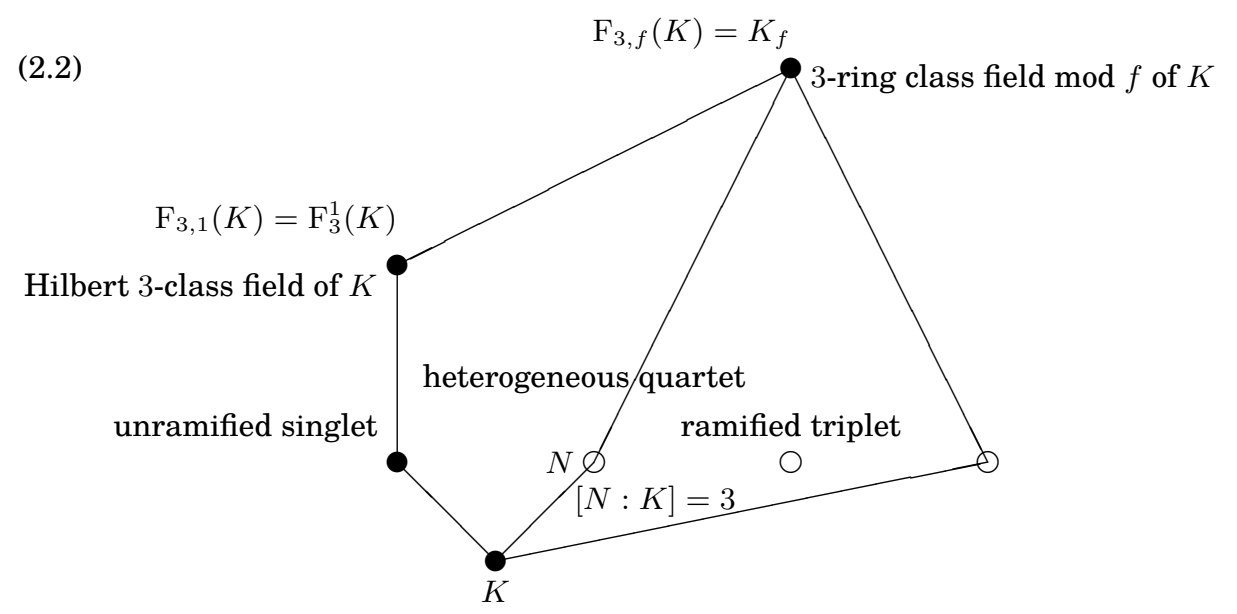

Figure 9: Heterogeneous quartet modulo $f=19$ over $K$.

Theorem 9.4. (Verification of Conjecture 9.2, (2.1), and Conjecture 9.1, (2); see Figure 10)

There exist non-Galois totally real cubic fields $L$ whose Galois closure $N$ is ramified, with conductor $f>1$ divisible only by prime divisors which do not split in $K$, i.e. $s=0$, over a real quadratic field $K$ with 3-class rank $\varrho_{3}(K)=2$, with twodimensional capitulation of the elementary 3-class group in $N$, and such that $U_{N}=U_{0}$. The minimal discriminant of such a field $L$ is

$$
d_{L}=\mathbf{1 8 2 5 1 0 6 0}=2^{2} \cdot 4562765
$$

(the first five fields of a nonet $\left(\alpha_{1}, \alpha_{1}, \alpha_{1}, \alpha_{1}, \alpha_{1}, \beta_{1}, \delta_{1}, \delta_{1}, \delta_{1}\right)$, discovered 23 November 2017).

Proof. The proof is conducted in the following Section 9.2.

The proof of Theorem 9.3 and Theorem 9.4 is conducted in the following sections on real quadratic base fields with 3-class rank 1 and 2. 


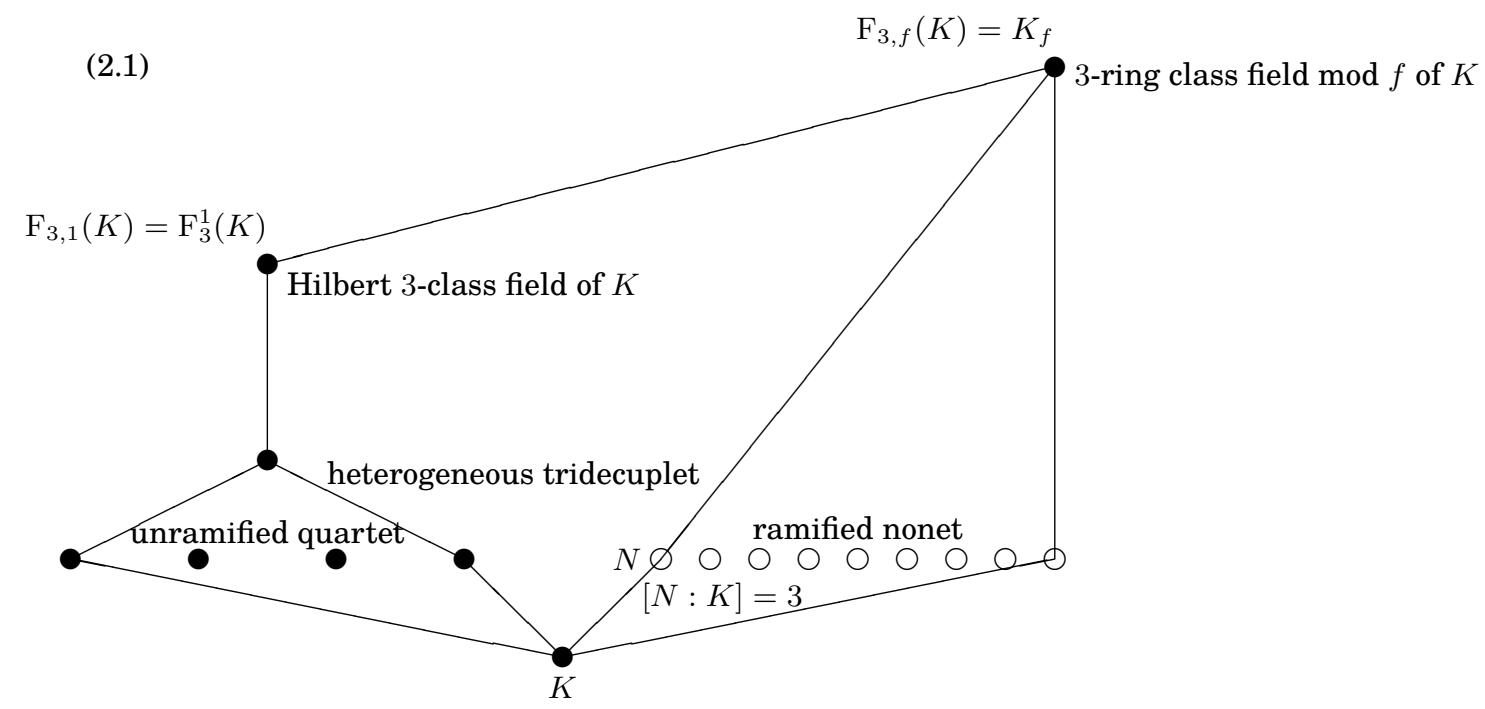

Figure 10: Heterogeneous tridecuplet modulo $f=2$ over $K$.

\subsection{Real quadratic base fields with 3-class rank 1}

In Table 29, we present the results of our search for the minimal discriminant $d_{L}$, respectively $d_{N}$, of a non-Galois totally real cubic field $L$, respectively its normal closure $N$, with differential principal factorization type $\alpha_{2}$. Since $\varrho=1$, the unramified component is a singlet, which must be of DPF type $\delta_{1}$. Since $t=s=1$, the DPF types $\alpha_{2}, \beta_{1}, \beta_{2}, \delta_{1}, \delta_{2}, \varepsilon$ would be possible, for each member of the ramified triplet, but only the types $\alpha_{2}, \delta_{1}, \delta_{2}$ occur usually.

The desired minimum is clearly given by $d_{L}=19^{2} \cdot 2677=966397$ with two occurrences of ramified extensions having DPF type $\alpha_{2}$. For $f=3^{2}$, the condition $d \equiv 1(\bmod 3)$ is required.

Table 29: Heterogeneous quartets of $S_{3}$-fields with splitting prime (power) $f$.

\begin{tabular}{|r|r|r||c||ccc|}
\hline & & \multicolumn{1}{|c||}{} & unramified component & \multicolumn{3}{|c|}{ ramified components } \\
$f$ & $d$ & $d_{L}=f^{2} \cdot d$ & $\delta_{1}$ & $\alpha_{2}$ & $\delta_{1}$ & $\delta_{2}$ \\
\hline $3^{2}$ & 14197 & 1149957 & 1 & 3 & 0 & 0 \\
7 & 21781 & 1067269 & 1 & 2 & 1 & 0 \\
13 & 9749 & 1647581 & 1 & 2 & 0 & 1 \\
19 & 2677 & $\mathbf{9 6 6 3 9 7}$ & 1 & 2 & 1 & 0 \\
31 & 3877 & 3725797 & 1 & 2 & 0 & 1 \\
37 & 5477 & 7498013 & 1 & 1 & 0 & 2 \\
43 & 4933 & 9121117 & 1 & 3 & 0 & 0 \\
61 & 3981 & 14813301 & 1 & 3 & 0 & 0 \\
67 & 4493 & 20169077 & 1 & 2 & 0 & 1 \\
73 & 10733 & 57196157 & 1 & 3 & 0 & 0 \\
\hline
\end{tabular}

Since we know a small candidate $d_{L}=966397$ for the minimal discriminant, and since the smallest quadratic fundamental discriminant with $\varrho=1$ is $d=229$, we only have to investigate prime and composite conductors $f=\sqrt{\frac{d_{L}}{d_{K}}}$ with $s \geq 1$ and

$$
f \leq \sqrt{\frac{966397}{229}} \approx \sqrt{4220} \approx 64.9
$$

which are divisible by a split prime, that is,

$$
\begin{gathered}
f \in\left\{7,9=3^{2}, 13,14=2 \cdot 7,18=2 \cdot 3^{2}, 19,21=3 \cdot 7,26=2 \cdot 13,31,35=5 \cdot 7,37,\right. \\
\left.38=2 \cdot 19,39=3 \cdot 13,42=2 \cdot 3 \cdot 7,43,45=5 \cdot 3^{2}, 57=3 \cdot 19,61,62=2 \cdot 31,63=7 \cdot 3^{2}\right\} .
\end{gathered}
$$

The result of the investigations is summarized in Table 30, which clearly shows that $d_{L}=\mathbf{9 6 6 3 9 7}$, for $d=2677$ and splitting prime conductor $f=19$ bigger than the conductor $f=1$ of unramified extensions $N / K$, is the desired minimal discriminant of a totally real cubic field with ramified extension $N / K$, DPF type $\alpha_{2}$ and $U_{N}=U_{0}$. The information has been computed with Fieker's class field theoretic routines of Magma [11,25]. 
Table 30: Heterogeneous quartets of $S_{3}$-fields with conductor $f$, where $s=1$.

\begin{tabular}{|c|c|c|c|c|c|c|c|c|c|}
\hline \multirow[b]{2}{*}{$f$} & \multirow[b]{2}{*}{ condition } & \multirow[b]{2}{*}{$d$} & \multirow[b]{2}{*}{$d_{L}=f^{2} \cdot d$} & \multirow{2}{*}{$\begin{array}{l}\text { unramified component } \\
\delta_{1}\end{array}$} & \multicolumn{5}{|c|}{ ramified components } \\
\hline & & & & & $\alpha_{2}$ & $\beta_{1}$ & $\beta_{2}$ & $\delta_{1}$ & $\delta_{2}$ \\
\hline 7 & & 21781 & 1067269 & 1 & 2 & 0 & 0 & 1 & 0 \\
\hline $3^{2}$ & $d \equiv 1(3)$ & 14197 & 1149957 & 1 & 3 & 0 & 0 & 0 & 0 \\
\hline 13 & & 9749 & 1647581 & 1 & 2 & 0 & 0 & 0 & 1 \\
\hline 19 & & 2677 & 966397 & 1 & 2 & 0 & 0 & 1 & 0 \\
\hline 31 & & 3877 & 3725797 & 1 & 2 & 0 & 0 & 0 & 1 \\
\hline 37 & & 5477 & 7498013 & 1 & 1 & 0 & 0 & 0 & 2 \\
\hline 43 & & 4933 & 9121117 & 1 & 3 & 0 & 0 & 0 & 0 \\
\hline 61 & & 3981 & 14813301 & 1 & 3 & 0 & 0 & 0 & 0 \\
\hline $2 \cdot 7$ & & 6997 & 1371412 & 1 & 3 & 0 & 0 & 0 & 0 \\
\hline $2 \cdot 3^{2}$ & $d \equiv 1(3)$ & 16141 & 5229684 & 1 & 3 & 0 & 0 & 0 & 0 \\
\hline $3 \cdot 7$ & $d \equiv 3(9)$ & 28137 & 12408417 & 1 & 3 & 0 & 0 & 0 & 0 \\
\hline $3 \cdot 7$ & $d \equiv 6(9)$ & 57516 & 25364556 & 1 & 3 & 0 & 0 & 0 & 0 \\
\hline $2 \cdot 13$ & & 21557 & 14572532 & 1 & 3 & 0 & 0 & 0 & 0 \\
\hline $5 \cdot 7$ & & 14457 & 17709825 & 1 & 3 & 0 & 0 & 0 & 0 \\
\hline $2 \cdot 19$ & & 13765 & 19876660 & 1 & 3 & 0 & 0 & 0 & 0 \\
\hline $3 \cdot 13$ & $d \equiv 3(9)$ & 51528 & 78374088 & 1 & 3 & 0 & 0 & 0 & 0 \\
\hline $3 \cdot 13$ & $d \equiv 6(9)$ & 37176 & 56544696 & 1 & 3 & 0 & 0 & 0 & 0 \\
\hline $2 \cdot 3 \cdot 7$ & $d \equiv 3(9)$ & 891237 & 1572142068 & 1 & 4 & 1 & 1 & 0 & 0 \\
\hline $2 \cdot 3 \cdot 7$ & $d \equiv 6(9)$ & 474261 & 836596404 & 1 & 2 & 0 & 1 & 0 & 0 \\
\hline $5 \cdot 3^{2}$ & $d \equiv 1(3)$ & 24952 & 50527800 & 1 & 1 & 0 & 0 & 1 & 1 \\
\hline $3 \cdot 19$ & $d \equiv 3(9)$ & 24393 & 79252857 & 1 & 3 & 0 & 0 & 0 & 0 \\
\hline $3 \cdot 19$ & $d \equiv 6(9)$ & 39417 & 128065833 & 1 & 3 & 0 & 0 & 0 & 0 \\
\hline $2 \cdot 31$ & & 7573 & 29110612 & 1 & 3 & 0 & 0 & 0 & 0 \\
\hline $7 \cdot 3^{2}$ & $d \equiv 1(3)$ & 2941 & 11672829 & 1 & 3 & 0 & 0 & 0 & 0 \\
\hline $7 \cdot 3^{2}$ & $d \equiv 2(3)$ & 23993 & 95228217 & 1 & 3 & 0 & 0 & 0 & 0 \\
\hline
\end{tabular}

\subsection{Real quadratic base fields with 3-class rank 2}

In this situation, the unramified quartet is non-trivial, since two DPF types $\alpha_{1}$ and $\delta_{1}$ are possible. These quartets have been thoroughly studied in [29], and in Table 31 and 32, we use the corresponding notation for capitulation types.

In Table 31, we present the results of the crucial search for the minimal discriminant $d_{L}$, respectively $d_{N}$, of a nonGalois totally real cubic field $L$, respectively its normal closure $N$, with differential principal factorization type $\alpha_{1}$ such that $N / K$ is a ramified extension of a real quadratic field $K$ with 3-class rank $\varrho=2$. We tried to fix the minimal possible conductor $f>1$, namely $f=2$. This experiment was motivated by the fact that the conductor $f$ enters the expression $d_{L}=f^{2} \cdot d$ in its second power, whereas the quadratic discriminant $d$ enters linearly. Consequently, the probability to find the minimum of $d_{L}$ is higher for small $f$ than for small $d$.

The table is ordered by increasing quadratic fundamental discriminants $d$ and gives $d_{L}=2^{2} \cdot d$ and the Artin pattern $(\varkappa, \tau)$ of the heterogeneous tridecuplet of cyclic cubic relative extensions $N / K$ consisting of an unramified quartet $\left(N_{1,1}, \ldots, N_{1,4}\right)$ with conductor $f^{\prime}=1$ and a ramified nonet $\left(N_{2,1}, \ldots, N_{2,9}\right)$ with conductor $f=2$, grouped by the possible two, respectively four, DPF types $\alpha_{1}, \delta_{1}$, respectively $\alpha_{1}, \beta_{1}, \delta_{1}, \varepsilon$. Transfer kernels $\varkappa$ are abbreviated by digits, 0 for twodimensional and $1, \ldots, 4$ for one-dimensional principalization, and an asterisk $*$ for a trivial kernel. Transfer targets $\tau$ are abbreviated by logarithmic Abelian type invariants of 3-class groups. Symbolic exponents always denote iteration.

The desired minimum is given by $d_{L}=4 \cdot 4562765=\mathbf{1 8 2 5 1 0 6 0}$ with five occurrences of ramified extensions with DPF type $\alpha_{1}$. Generally, there is an abundance of ramified extensions with two-dimensional capitulation kernel: at least three and at most all nine of a nonet.

Table 32 shows analogous results for the conductor $f=5$, that is, $d_{L}=5^{2} \cdot d$. The minimum $d_{L}=25 \cdot 1049512=26237800$ is clearly beaten by the minimum $4 \cdot 4562765=18251060$ in Table 31 .

Since we know a small candidate $d_{L}=18251060$ for the minimal discriminant, and since the smallest quadratic discriminant with $\varrho=2$ is $d=32009$, we only have to investigate prime and composite conductors $f=\sqrt{\frac{d_{L}}{d_{K}}}$ with

$$
f \leq \sqrt{\frac{18251060}{32009}} \approx \sqrt{570.2} \approx 23.9
$$


Table 31: Artin pattern $(\varkappa, \tau)$ of heterogeneous multiplets modulo $f=2$.

\begin{tabular}{|c|c|c|c|c|c|c|c|c|c|c|c|c|c|}
\hline \multirow[b]{3}{*}{$d_{K}$} & \multicolumn{5}{|c|}{ unramified components } & \multicolumn{8}{|c|}{ ramified components } \\
\hline & \multirow[b]{2}{*}{ Type } & \multicolumn{2}{|c|}{$\alpha_{1}$} & \multicolumn{2}{|c|}{$\delta_{1}$} & \multicolumn{2}{|r|}{$\alpha_{1}$} & \multicolumn{2}{|c|}{$\beta_{1}$} & \multicolumn{2}{|c|}{$\delta_{1}$} & \multicolumn{2}{|c|}{$\varepsilon$} \\
\hline & & $\varkappa$ & $\tau$ & $\varkappa$ & $\tau$ & $\varkappa$ & $\tau$ & $\varkappa$ & $\tau$ & $\varkappa$ & $\tau$ & $\varkappa$ & $\tau$ \\
\hline 4562765 & a. $3^{*}$ & $0^{3}$ & $\left(1^{2}\right)^{3}$ & 1 & $1^{3}$ & $0^{5}$ & $2^{2} 1^{2},\left(1^{4}\right)^{4}$ & 1 & $1^{5}$ & $14^{2}$ & $\left(21^{3}\right)^{3}$ & & \\
\hline 7339397 & a. $3^{*}$ & $0^{3}$ & $\left(1^{2}\right)^{3}$ & 1 & $1^{3}$ & $0^{7}$ & $\left(1^{4}\right)^{7}$ & 2 & $21^{3}$ & 1 & $21^{3}$ & & \\
\hline 7601461 & a. 3 & $0^{3}$ & $\left(1^{2}\right)^{3}$ & 1 & 21 & $0^{6}$ & $2^{2} 1^{2},\left(1^{4}\right)^{5}$ & & & 234 & $\left(21^{3}\right)^{3}$ & & \\
\hline 7657037 & a. 3 & $0^{3}$ & $\left(1^{2}\right)^{3}$ & 1 & 21 & $0^{6}$ & $\left(1^{4}\right)^{6}$ & 1 & $21^{3}$ & 12 & $1^{5}, 21^{3}$ & & \\
\hline 7736749 & a. $3^{*}$ & $0^{3}$ & $\left(1^{2}\right)^{3}$ & 1 & $1^{3}$ & $0^{7}$ & $\left(1^{4}\right)^{7}$ & & & $4^{2}$ & $\left(21^{3}\right)^{2}$ & & \\
\hline 8102053 & a. $3^{*}$ & $0^{3}$ & $\left(1^{2}\right)^{3}$ & 1 & $1^{3}$ & $0^{7}$ & $\left(1^{4}\right)^{7}$ & & & 23 & $1^{5}, 21^{3}$ & & \\
\hline 9182229 & a. 2 & $0^{3}$ & $\left(1^{2}\right)^{3}$ & 4 & 21 & $0^{8}$ & $2^{2} 1^{2},\left(1^{4}\right)^{7}$ & & & 2 & $21^{3}$ & & \\
\hline 9500453 & a. 3 & $0^{3}$ & $\left(1^{2}\right)^{3}$ & 1 & 21 & $0^{8}$ & $2^{2} 1^{2},\left(1^{4}\right)^{7}$ & & & 3 & $21^{3}$ & & \\
\hline 9533357 & a. 3 & $0^{3}$ & $\left(1^{2}\right)^{3}$ & 1 & 21 & $0^{6}$ & $\left(1^{4}\right)^{6}$ & 1 & $21^{3}$ & 23 & $\left(21^{3}\right)^{2}$ & & \\
\hline 11003845 & a. 3 & $0^{3}$ & $\left(1^{2}\right)^{3}$ & 1 & 21 & $0^{4}$ & $\left(1^{4}\right)^{4}$ & & & $12^{2} 4^{2}$ & $1^{5},\left(21^{3}\right)^{4}$ & & \\
\hline 12071253 & a. 3 & $0^{3}$ & $\left(1^{2}\right)^{3}$ & 1 & 21 & $0^{7}$ & $\left(1^{4}\right)^{7}$ & 3 & $21^{3}$ & 2 & $21^{3}$ & & \\
\hline 14266853 & a.3 & $0^{3}$ & $\left(1^{2}\right)^{3}$ & 1 & 21 & $0^{8}$ & $2^{2} 1^{2},\left(1^{4}\right)^{7}$ & & & 4 & $21^{3}$ & & \\
\hline 14308421 & a. $3^{*}$ & $0^{3}$ & $\left(1^{2}\right)^{3}$ & 1 & $1^{3}$ & $0^{4}$ & $\left(1^{4}\right)^{4}$ & & & $1^{2} 234$ & $2^{3} 1,\left(21^{3}\right)^{3}, 1^{5}$ & & \\
\hline 14315765 & a. 3 & $0^{3}$ & $\left(1^{2}\right)^{3}$ & 1 & 21 & $0^{7}$ & $\left(1^{4}\right)^{7}$ & & & 23 & $\left(21^{3}\right)^{2}$ & & \\
\hline 14395013 & a. $3^{*}$ & $0^{3}$ & $\left(1^{2}\right)^{3}$ & 1 & $1^{3}$ & $0^{6}$ & $\left(1^{4}\right)^{6}$ & 1 & $21^{3}$ & 23 & $\left(21^{3}\right)^{2}$ & & \\
\hline 15131149 & D.10 & & & 2414 & $(21)^{3}, 1^{3}$ & $0^{7}$ & $\left(1^{4}\right)^{7}$ & 1 & $21^{3}$ & 1 & $21^{3}$ & & \\
\hline 16385741 & a. $3^{*}$ & $0^{3}$ & $\left(1^{2}\right)^{3}$ & 1 & $1^{3}$ & $0^{4}$ & $\left(1^{4}\right)^{4}$ & & & $23^{2} 4$ & $\left(21^{3}\right)^{4}$ & $*$ & $32^{2} 1$ \\
\hline
\end{tabular}

Table 32: Artin pattern $(\varkappa, \tau)$ of heterogeneous multiplets modulo $f=5$.

\begin{tabular}{|c|c|c|c|c|c|c|c|c|c|c|c|c|c|}
\hline \multirow[b]{3}{*}{$d_{K}$} & \multicolumn{5}{|c|}{ unramified components } & \multicolumn{8}{|c|}{ ramified components } \\
\hline & & & $\alpha_{1}$ & & $\delta_{1}$ & & $\alpha_{1}$ & & $\beta_{1}$ & & 1 & & $\varepsilon$ \\
\hline & Type & $\varkappa$ & $\tau$ & $\varkappa$ & $\tau$ & 2 & $\tau$ & $\varkappa$ & $\tau$ & $\varkappa$ & $\tau$ & $\varkappa$ & $\tau$ \\
\hline 1049512 & a. 3 & & $\left(1^{2}\right)^{3}$ & 1 & 21 & 0 & $\left(1^{4}\right)^{4}$ & & & $234^{3}$ & $\left(21^{3}\right)^{5}$ & & \\
\hline 2461537 & a. 2 & & $\left(1^{2}\right)^{3}$ & 4 & 21 & 0 & $\left(1^{4}\right)^{7}$ & & & 12 & $\left(21^{3}\right)^{2}$ & & \\
\hline 2811613 & a. $3^{*}$ & $0^{3}$ & $\left(1^{2}\right)^{3}$ & 1 & $1^{3}$ & 0 & $2^{2} 1^{2},\left(1^{4}\right)^{4}$ & 2 & $21^{3}$ & 123 & $\left(21^{3}\right)^{3}$ & & \\
\hline 3091133 & a. 3 & $0^{3}$ & $\left(1^{2}\right)^{3}$ & 1 & 21 & 0 & $\left(1^{4}\right)^{4}$ & 4 & $21^{3}$ & $1^{3} 2$ & $\left(21^{3}\right)^{4}$ & & \\
\hline 5858753 & G.19 & & & 2143 & $(21)^{4}$ & 0 & $\left(2^{2} 1^{2}\right)^{3},\left(1^{4}\right)^{4}$ & & & 3 & $21^{3}$ & & $21^{4}$ \\
\hline 6036188 & D. 10 & & & 3431 & $1^{3},(21)^{3}$ & 0 & $\left(1^{4}\right)^{8}$ & & & & & & $2^{2} 1^{2}$ \\
\hline
\end{tabular}

that is,

$$
\begin{gathered}
f \in\left\{2,3,5,6=2 \cdot 3,7,9=3^{2}, 10=2 \cdot 5,11,13,14=2 \cdot 7,\right. \\
\left.15=3 \cdot 5,17,18=2 \cdot 3^{2}, 19,21=3 \cdot 7,22=2 \cdot 11,23\right\} .
\end{gathered}
$$

The result of the investigations is summarized in Table 33, which clearly shows that $d_{L}=\mathbf{1 8 2 5 1 0 6 0}$, for $d=4562765$ and the smallest possible conductor $f=2$ bigger than the conductor $f=1$ of unramified extensions $N / K$, is the desired minimal discriminant of a totally real cubic field with ramified extension $N / K$, DPF type $\alpha_{1}$ and $U_{N}=U_{0}$. The information has been computed with Fieker's class field theoretic routines of Magma [11,25].

\subsection{Scholz conjecture for $p \geq 5$}

We have been curious if the conjecture of Scholz can also be verified for dihedral fields $N / \mathbb{Q}$ of degrees 10 and 14 . This is indeed the case, and the root discriminants $f^{2} \cdot d$ in the following theorem are probably minimal.

Theorem 9.5. Let $p$ be an odd prime number. Suppose Lis a non-Galois number field of degree $p$ with totally real absolutely dihedral Galois closure $N$ of degree $2 p$, and let $K$ be the unique real quadratic subfield of $N$. Then $N$ satisfies the condition $U_{N}=U_{0}:=\left\langle U_{K}, U_{L}, U_{L^{(1)}}, \ldots, U_{L^{(p-1)}}\right\rangle$,

1. if $d_{L}=\left(f^{2} \cdot d\right)^{2}$ with $f=11 \cdot 31, d=5, f^{2} \cdot d=581405$, when $p=5$,

2. if $d_{L}=\left(f^{2} \cdot d\right)^{3}$ with $f=29 \cdot 43, d=13, f^{2} \cdot d=20215117$, when $p=7$. 
Table 33: Heterogeneous tridecuplets of $S_{3}$-fields with conductor $f$.

\begin{tabular}{|c|c|c|c|c|c|c|c|c|c|}
\hline \multirow[b]{2}{*}{$f$} & \multirow[b]{2}{*}{ condition } & \multirow[b]{2}{*}{$d$} & \multirow[b]{2}{*}{$d_{L}=f^{2} \cdot d$} & \multicolumn{2}{|c|}{ unramified components } & \multicolumn{4}{|c|}{ ramified components } \\
\hline & & & & $\alpha_{1}$ & $\delta_{1}$ & $\alpha_{1}$ & $\beta_{1}$ & $\delta_{1}$ & $\varepsilon$ \\
\hline 2 & & 4562765 & 18251060 & 3 & 1 & 5 & 1 & 3 & 0 \\
\hline 3 & $d \equiv 3(9)$ & 9964821 & 89683389 & 3 & 1 & 4 & 0 & 4 & 1 \\
\hline 5 & & 1049512 & 26237800 & 3 & 1 & 4 & 0 & 5 & 0 \\
\hline 7 & & 966053 & 47336597 & 3 & 1 & 4 & 0 & 4 & 1 \\
\hline $3^{2}$ & $d \equiv 1(3)$ & 1482568 & 120088008 & 3 & 1 & 5 & 1 & 2 & 1 \\
\hline $3^{2}$ & $d \equiv 2(3)$ & 2515388 & 203746428 & 3 & 1 & 6 & 1 & 2 & 0 \\
\hline $3^{2}$ & $d \equiv 6(9)$ & 621429 & 50335749 & 3 & 1 & 6 & 0 & 3 & 0 \\
\hline 11 & & 476152 & 57614392 & 3 & 1 & 7 & 0 & 2 & 0 \\
\hline 13 & & 1122573 & 189714837 & 3 & 1 & 7 & 0 & 2 & 0 \\
\hline 17 & & 665832 & 192425848 & 3 & 1 & 7 & 0 & 2 & 0 \\
\hline 19 & & 635909 & 229563149 & 3 & 1 & 5 & 3 & 1 & 0 \\
\hline 23 & & 390876 & 206773404 & 3 & 1 & 7 & 1 & 1 & 0 \\
\hline $2 \cdot 3$ & $d \equiv 3(9)$ & 5963493 & 214685748 & 3 & 1 & 7 & 2 & 0 & 0 \\
\hline $2 \cdot 3$ & $d \equiv 6(9)$ & 4305957 & 155014452 & 0 & 4 & 6 & 3 & 0 & 0 \\
\hline $2 \cdot 5$ & & 363397 & 36339700 & 3 & 1 & 6 & 3 & 0 & 0 \\
\hline $2 \cdot 7$ & & 358285 & 70223860 & 4 & 0 & 7 & 2 & 0 & 0 \\
\hline $3 \cdot 5$ & $d \equiv 3(9)$ & 4845432 & 1090222200 & 3 & 1 & 6 & 3 & 0 & 0 \\
\hline $3 \cdot 5$ & $d \equiv 6(9)$ & 1646817 & 370533825 & 3 & 1 & 6 & 3 & 0 & 0 \\
\hline $2 \cdot 3^{2}$ & $d \equiv 1(3)$ & 2142445 & 694152180 & 3 & 1 & 6 & 3 & 0 & 0 \\
\hline $2 \cdot 3^{2}$ & $d \equiv 2(3)$ & 635909 & 206034516 & 3 & 1 & 6 & 3 & 0 & 0 \\
\hline $2 \cdot 3^{2}$ & $d \equiv 6(9)$ & 2538285 & 822404340 & 3 & 1 & 6 & 3 & 0 & 0 \\
\hline $3 \cdot 7$ & $d \equiv 3(9)$ & 3597960 & 1586700360 & 3 & 1 & 6 & 3 & 0 & 0 \\
\hline $3 \cdot 7$ & $d \equiv 6(9)$ & 3122232 & 1376904312 & 0 & 4 & 6 & 3 & 0 & 0 \\
\hline $2 \cdot 11$ & & 2706373 & 1309884532 & 3 & 1 & 6 & 3 & 0 & 0 \\
\hline
\end{tabular}

In both cases, the conductor is of the form $f=\ell_{1} \cdot \ell_{2}$ with prime numbers $\ell_{i} \equiv+1(\bmod p)$ which split in $K$, and L is a singlet with differential principal factorization type $\tau(L)=\alpha_{3}$.

Proof. By immediate inspection of real quadratic fields $K$ with $p$-class rank $\varrho_{p}=0$ and $p$-admissible conductors $f$, divisible by two primes which split in $K$, with the aid of Magma.

\section{Conclusion}

In this paper, we have given the complete classification of all multiplets of totally real cubic fields $L$ in the range $0<d_{L}<10^{7}$ of Llorente and Quer [24] according to their differential principal factorizations (Tables 13 and 14). Inspired by discussions after our two presentations at the West Coast Number Theory Conference in Asilomar, December 1990, we had attempted this classification in August 1991 already, but we were forced to restrict the range to the upper bound $2 \cdot 10^{5}$ in [27]. In spite of the required correction of 14 errors (Tables 8 and 9), the table [27] and the associated theory [26], thirty years ago, were a progressive innovation concerning DPF types of multiplets of dihedral fields, and a prototype for the present paper and its predecessor [35].

We have also given the complete verification of the Conjecture of Arnold Scholz (Conjecture 9.1). It was necessary to develop the new concept of relative principal factorizations in order to illuminate the full reach of this conjecture, which we have reformulated more ostensively in Conjecture 9.2. Due to the computational challenges, the proof of each of the different perspectives of the conjecture was established many years after Scholz's paper in 1933 [39]: Corollary 9.1 on $f=1$ was proved 49 years later in 1982 [17], Theorem 9.2 concerning the type $\alpha_{3}$ singulet 58 years later in 1991 [27], Theorem 9.3 on the triplet containing type $\alpha_{2}$ even 84 years later on 19 November 2017, and Theorem 9.4 on the nonet containing type $\alpha_{1}$ with $f>1$ also 84 years later on 23 November 2017.

In our ultimate Table 34, we emphasize the apparent asymptotic tendencies of DPF types, based on five ranges of discriminants $0<d_{L}<B$ with increasing upper bounds $B$. Relative frequencies are rounded to integer percentages. It is striking that the normal closures $N$ of an overwhelming proportion with $93 \%$ of all totally real cubic fields $L$ have a unit group $U_{N}$ which is a non-split extension of $U_{K}=\langle-1, \eta\rangle$ if considered as a module over the integral group algebra $\mathbb{Z}\left[S_{3}\right]$, 
since it contains a unit $H$ such that $N_{N / K}(H)=H \cdot H^{\sigma} \cdot H^{\sigma^{2}}=\eta$, according to Remark 3.3.

This phenomenon is due to extremely dominating unramified extensions $N / K$ with conductor $f=1, \varrho=1$, and mandatory type $\delta_{1}(72 \%)$, and ramified extensions $N / K$ with regular prime(power) conductor $f, \varrho=0$, and mandatory type $\varepsilon(21 \%)$. In contrast, the contributions by the rare types $\alpha_{2}$ and $\alpha_{3}$ and by the cyclic cubic fields $\zeta$ are in fact negligible. In spite of its distinctive dominance for conductors with two or more prime divisors, type $\gamma$ remains marginal with $2 \%$. Other marginal (but not negligible) contributions arise from type $\alpha_{1}$, due to increasing occurrences of $\varrho=2$, from type $\beta_{1}$, due to capitulation in ramified extensions with $\varrho=1$, and from the types $\beta_{2}$ and $\delta_{2}$, due to conductors $f$ with a prime divisor which splits in the quadratic subfield $K<N$.

Table 34: Tendencies of the statistical distribution of DPF types.

\begin{tabular}{|c||rr||rr||rr||rr||rr|}
\hline \multicolumn{1}{|c||}{$B$} & \multicolumn{1}{c||}{1500} & \multicolumn{2}{c||}{$10^{5}$} & \multicolumn{2}{c||}{$2 \cdot 10^{5}$} & $5 \cdot 10^{5}$ & $10^{7}$ \\
Type & $\#$ & $\%$ & $\#$ & $\%$ & $\#$ & $\%$ & $\#$ & $\%$ & $\#$ & $\%$ \\
\hline$\alpha_{1}$ & 0 & 0 & 16 & 0 & 50 & 1 & 175 & 1 & 7951 & 1 \\
$\alpha_{2}$ & 0 & 0 & 0 & 0 & 0 & 0 & 0 & 0 & 142 & 0 \\
$\alpha_{3}$ & 0 & 0 & 0 & 0 & 1 & 0 & 3 & 0 & 122 & 0 \\
$\beta_{1}$ & 0 & 0 & 10 & 0 & 21 & 0 & 89 & 0 & 3924 & 1 \\
$\beta_{2}$ & 0 & 0 & 76 & 2 & 155 & 2 & 380 & 1 & 7639 & 1 \\
$\gamma$ & 2 & 4 & 106 & 2 & 201 & 2 & 493 & 2 & 9420 & 2 \\
\hline$\delta_{1}$ & 26 & 59 & 3349 & 70 & 7028 & 70 & 18714 & 71 & 426972 & 72 \\
$\delta_{2}$ & 0 & 0 & 79 & 2 & 188 & 2 & 490 & 2 & 11128 & 2 \\
$\varepsilon$ & 10 & 23 & 1117 & 23 & 2301 & 23 & 5986 & 23 & 125123 & 21 \\
\hline$\zeta$ & 6 & 14 & 51 & 1 & 70 & 1 & 110 & 0 & 501 & 0 \\
\hline
\end{tabular}

\section{Acknowledgment}

This research is supported by the Austrian Science Fund (FWF): projects J0497-PHY and P26008-N25.

\section{References}

[1] I. O. Angell, Table of totally real cubic fields, Math. Comp. (1975), 79 sheets of computer output deposited in UMT file.

[2] I. O. Angell, A table of totally real cubic fields, Math. Comp. 30 (1976) 184-187; MR 53 \#5528 (Review by D. Shanks, Math. Comp. 30 (1976), 670-673, RMT 32).

[3] S. Aouissi, D. C. Mayer, M. C. Ismaili, M. Talbi, A. Azizi, 3-rank of ambiguous class groups in cubic Kummer extensions, Period. Math. Hung. 81 (2020) $250-274$.

[4] P. Barrucand, H. Cohn, Remarks on principal factors in a relative cubic field, J. Number Theory 3 (1971) $226-239$.

[5] K. Belabas, A fast algorithm to compute cubic fields, Math. Comp. 66 (1997) 1213-1237.

[6] W. Bosma, J. Cannon, C. Playoust, The Magma algebra system. I. The user language, J. Symbolic Comput. 24 (1997) $235-265$.

[7] W. Bosma, J. J. Cannon, C. Fieker, A. Steels, Handbook of Magma Functions, Edition 2.26, Sydney, 2021.

[8] S. M. Chang, R. Foote, Capitulation in class field extensions of type ( $p, p)$, Canad. J. Math. 32 (1980) 1229-1243.

[9] V. Ennola, R. Turunen, Tables of totally real cubic fields, Math. Comp. (1983), computer output deposited in UMT file.

[10] V. Ennola, R. Turunen, On totally real cubic fields, Math. Comp. 44 (1985) 495-518.

[11] C. Fieker, Computing class fields via the Artin map, Math. Comp. 70 (2001) 1293-1303.

[12] F. Halter-Koch, Eine bemerkung über kubische einheiten, Arch. Math. 27 (1976) 593-595.

[13] H. Hasse, Bericht über neuere untersuchungen und probleme aus der theorie der algebraischen zahlkörper. Teil Ia: Beweise zu Teil I, Jber. der DMV 36 (1927) 233-311.

[14] H. Hasse, Arithmetische theorie der kubischen zahlkörper auf klassenkörpertheoretischer grundlage, Math. Zeitschr. 31 (1930) 565-582.

[15] H. Hasse, Die Einheitengruppe in einem total-reellen nicht-zyklischen kubischen Zahlkörper und im zugehörigen bikubischen Normalkörper, Arch. Math. 1 (1948) 42-46.

[16] H. Hasse, Die Einheitengruppe in einem total-reellen nicht-zyklischen kubischen Zahlkörper und im zugehörigen bikubischen Normalkörper, Miscellanea Academica Berolinensia 1 (1950) 1-24.

[17] F.-P. Heider, B. Schmithals, Zur Kapitulation der Idealklassen in unverzweigten primzyklischen Erweiterungen, J. Reine Angew. Math. 336 (1982) $1-25$.

[18] J. Herbrand, Sur les théorèmes du genre principal et des idéaux principaux, Abh. Math. Sem. Hamburg 9 (1932) 84-92.

[19] D. Hilbert, Die Theorie der algebraischen Zahlkörper, Jber. der DMV 4 (1897) 175-546.

[20] K. Iwasawa, A note on the group of units of an algebraic number field, J. Math. Pures Appl. 35 (1956) 189-192.

[21] M. P. Lee, Integral representations of dihedral groups of order 2p, Trans. Amer. Math. Soc. 110 (1964) 213-231.

[22] P. Llorente, A. V. Oneto, Cuerpos cúbicos, Cursos, Seminarios y Tesis del PEAM, No. 5, Univ. Zulia, Maracaibo, Venezuela, 1980.

[23] P. Llorente, A. V. Oneto, On the real cubic fields, Math. Comp. 39 (1982) 689-692.

[24] P. Llorente, J. Quer, On totally real cubic fields with discriminant $D<10^{7}$, Math. Comp. 50 (1988) 581-594.

[25] MAGMA Developer Group, MAGMA Computational Algebra System, Version 2.26-1, Univ. Sydney, Sydney 2021.

[26] D. C. Mayer, Classification of Dihedral Fields, Preprint, Department of Computer Science, Univ. of Manitoba, Winnipeg, 1991.

[27] D. C. Mayer, List of Discriminants $d_{L}<200000$ of Totally Real Cubic Fields L, Arranged According to Their Multiplicities $m$ and Conductors $f$, Computer Centre, Department of Computer Science, University of Manitoba, Winnipeg, 1991.

[28] D. C. Mayer, Multiplicities of dihedral discriminants, Math. Comp. 58 (1992) 831-847 and S55-S58.

[29] D. C. Mayer, The second p-class group of a number field, Int. J. Number Theory 8 (2012) 471-505. 
[30] D. C. Mayer, The distribution of second p-class groups on coclass graphs, J. Théor. Nombres Bordeaux 25 (2013) $401-456$.

[31] D. C. Mayer, Quadratic p-ring spaces for counting dihedral fields, Int. J. Number Theory 10 (2014) 2205-2242.

[32] D. C. Mayer, Principalization algorithm via class group structure, J. Théor. Nombres Bordeaux 26 (2014) 415-464.

[33] D. C. Mayer, Differential principal factors and Pólya property of pure metacyclic fields, Int. J. Number Theory 15 (2019) $1983-2025$.

[34] D. C. Mayer, Generalized Artin pattern of heterogeneous multiplets of dihedral fields and proof of Scholz's conjecture, arXiv:1904.06148v1 [math.NT], (2019).

[35] D. C. Mayer, Construction and classification of $p$-ring class fields modulo $p$-admissible conductors, Open J. Math. Sci. 5 (2021) 162-171.

[36] N. Moser, Unités et nombre de classes d'une extension galoisienne diédrale de $\mathbb{Q}$, Abh. Math. Sem. Univ. Hamburg 48 (1979) 54-75.

[37] P. Satgé, Corps cubiques de discriminant donné, Acta Arith. 39 (1981) 295-301.

[38] B. Schmithals, Zur berechnung der kapitulation in bikubischen zahlkörpern, Manuscripta Math. 52 (1985) 171-202.

[39] A. Scholz, Idealklassen und einheiten in kubischen körpern, Monatsh. Math. Phys. 40 (1933) 211-222.

[40] G. F. Voronoi, Ob Odnom Obobshchenii Algorifma Nepreryvnykh Drobei, Doctoral Dissertation, Saint Petersburg State University, Saint Petersburg, 1896. 